\title{
PREDICTING BONE REMODELING IN RESPONSE TO ORTHOPEDIC IMPLANTATIONS: COMPUTATIONAL STUDY USING A MECHANO-BIOCHEMICAL MODEL
}

\author{
By: \\ Pouria Tavakkoli Avval \\ M.Sc. (Engineering Mechanics) \\ Royal Institute of Technology (Sweden), 2010 \\ A dissertation \\ presented to Ryerson University \\ in partial fulfillment of the requirements for \\ the degree of Doctor of Philosophy \\ in the Program of Mechanical Engineering
}

Toronto, Ontario, Canada, 2015

(C) Pouria Tavakkoli Avval, 2015 


\section{AUTHOR'S DECLARATION FOR ELECTRONIC SUBMISSION OF A DISSERTATION}

I hereby declare that I am the sole author of this dissertation.

I authorize Ryerson University to lend this dissertation to other institutions of individuals for the purpose of scholarly research.

I further authorize Ryerson University to reproduce this dissertation by photocopying or by other means, in total or in part, at the request of other institutions or individuals for the purpose of scholarly research. 


\section{ACKNOWLEDGEMENTS}

I would like to express my sincere gratitude to my instructors and research team mates who helped me complete this thesis study:

My supervisor, Dr. Habiba Bougherara, for her consistent guidance throughout the duration of this research. Without her guidance and encouragement, it would not have been possible for me to complete this study with absolute success.

Program Coordinator, Dr. Ahmad Ghasempoor for his guidance and support during my studies at Ryerson University.

Last, but certainly not least, I recognize my family, for their unconditional support in good and bad times. To you, I will always remain indebted. 
This page is intentionally left blank. 


\begin{abstract}
Predicting Bone Remodeling in Response to Orthopedic Implantations: Computational Study Using a Mechano-biochemical Model

\section{Pouria Tavakkoli Avval, M.Sc. (Engineering Mechanics)}

A dissertation presented to Ryerson University in partial fulfillment of the requirements for the degree of doctor of philosophy in the program of Mechanical Engineering
\end{abstract}

Toronto, Ontario, Canada, 2015

(C) Pouria Tavakkoli Avval, 2015

Periprosthetic bone loss following orthopedic implantations is a serious concern leading to the premature failure of the implants. Therefore, investigating bone remodeling in response to orthopedic implantations is of paramount importance for the purpose of designing long lasting prostheses. In this study, a predictive bone remodeling model (Thermodynamic-based model) was employed to simulate the long-term response of femoral density to total hip arthroplasty (THA), bone fracture plating and intramedullary (IM) nailing. The ability of the model in considering the coupling effect between mechanical loading and bone biochemistry is its unique characteristic. This research provided quantitative data for monitoring bone density changes throughout the femoral bone. The results obtained by the thermodynamic-based model agreed well with the bone morphology and the literature. The study revealed that the most significant periprosthetic bone loss in response to THA occurred in calcar region (Gruen zone 7). Conversely, the region beneath the hip stem (Gruen zone 4) experienced the lowest bone mineral density (BMD) changes. It was found that the composite hip implant and IM nail were more advantageous over the metallic ones as they induced less stress shielding and provided more uniform bone density changes following the surgery. The research study also showed that, due to plating, the areas beneath the bone fracture 
plate experienced severe bone loss. However, some level of bone formation was observed at the vicinity of the most proximal and distal screw holes in both lateral and anterior plated femurs. Furthermore, in terms of long-term density distributions, the anterior plating was not superior to the lateral plating. 


\section{TABLE OF CONTENTS}

AUTHOR'S DECLARATION FOR ELECTRONIC SUBMISSION OF A DISSERTATION ......ii

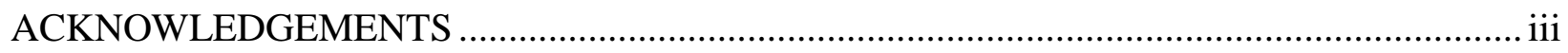

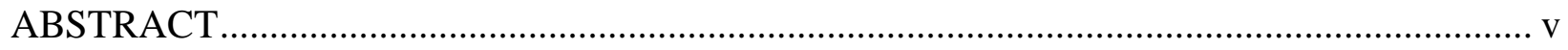

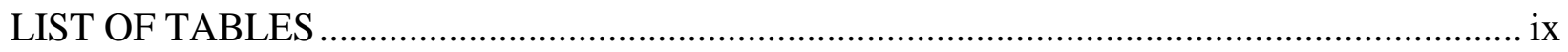

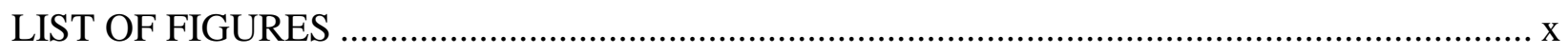

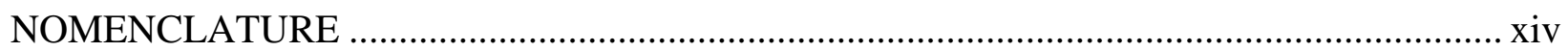

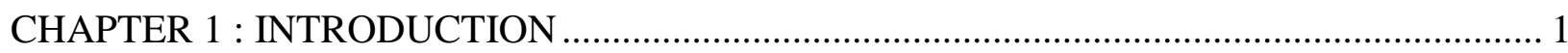

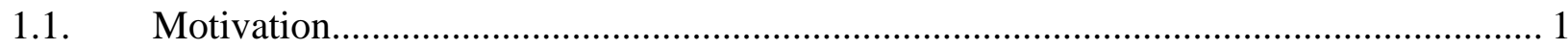

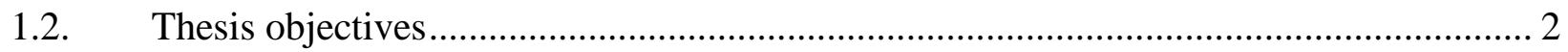

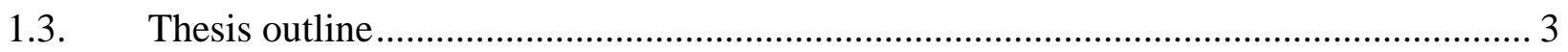

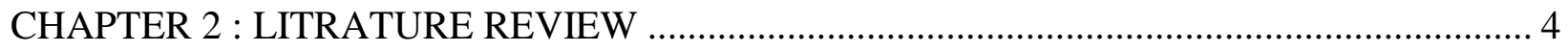

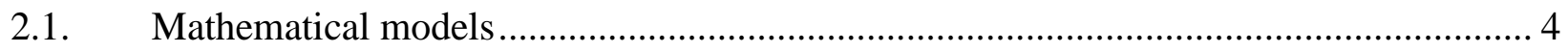

2.2. Early works on bone remodeling in response to composite implants ............................. 9

2.3. Early works on bone remodeling in response to hip implant and bone plate ................. 10

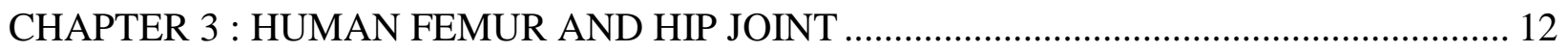

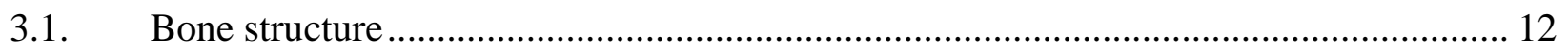

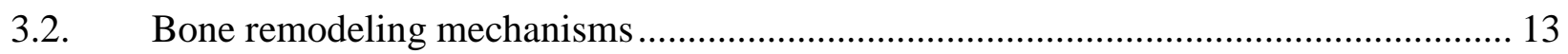

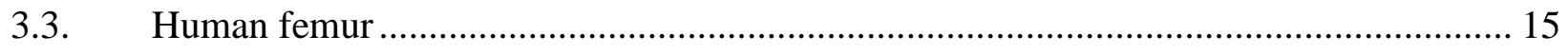

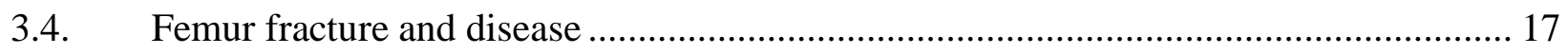

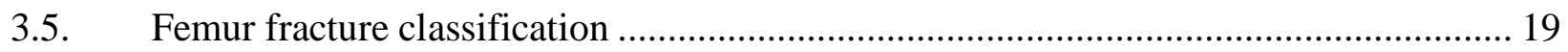

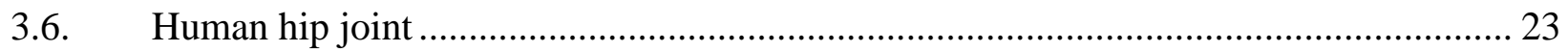

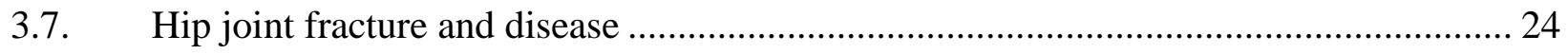

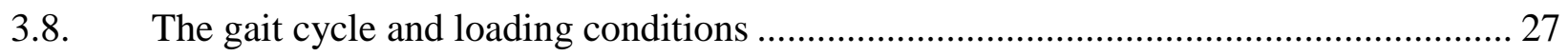




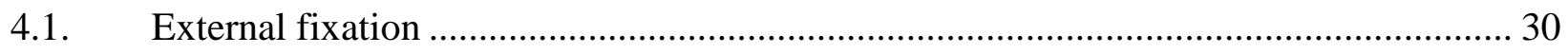

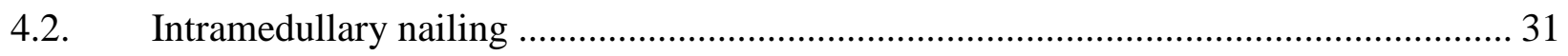

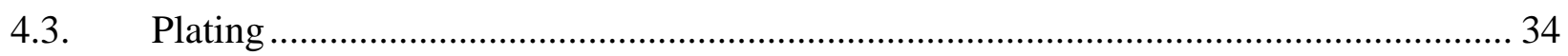

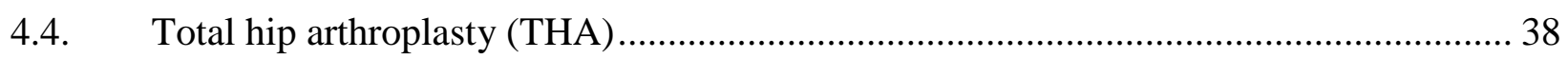

CHAPTER 5 : MATHEMATICAL FORMULATION OF THE MECHANO-BIOCHEMICAL MODEL (THERMODYNAMIC-BASED MODEL) .................................................... 41

5.1. Thermodynamic-based model (Mechano-biochemical model) ................................. 41

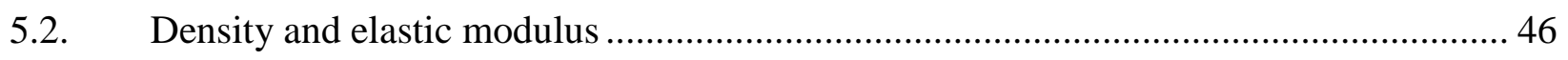

CHAPTER 6 : FINITE ELEMENT MODELING ............................................................. 47

6.1. Development of computer aided design (CAD) models....................................... 47

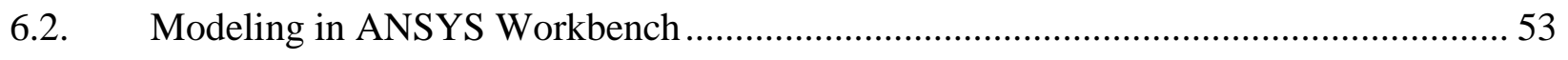

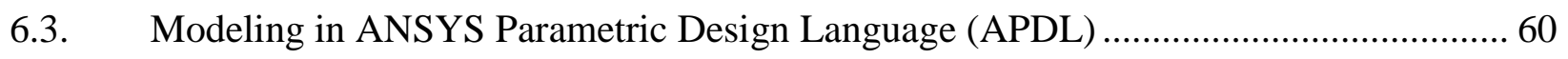

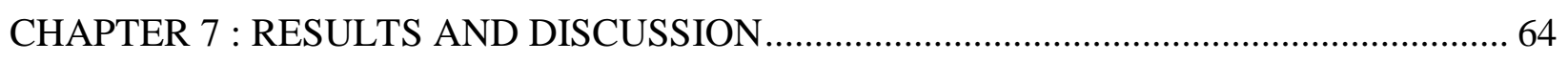

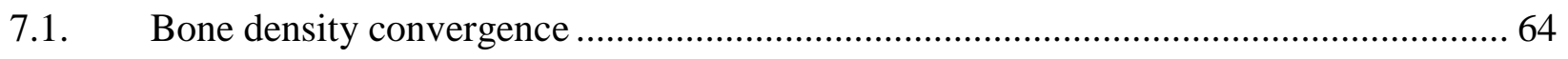

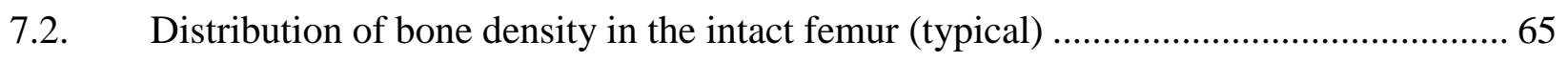

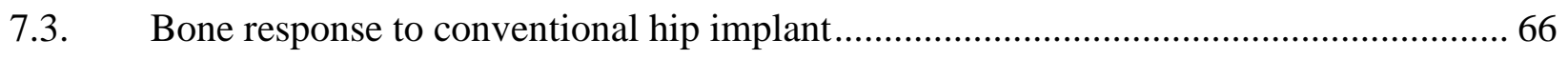

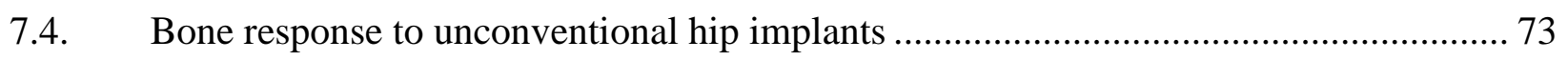

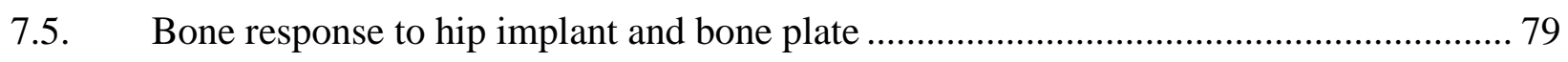

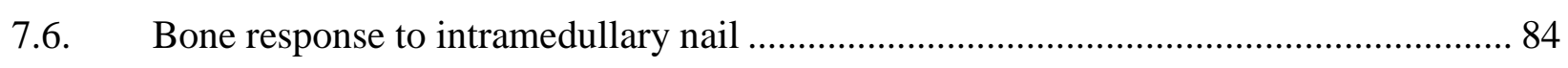

CHAPTER 8 : CONCLUSIONS, LIMITATIONS AND FUTURE WORK .......................... 89

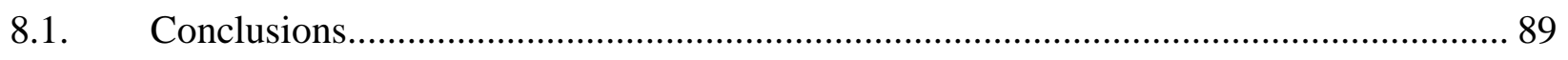

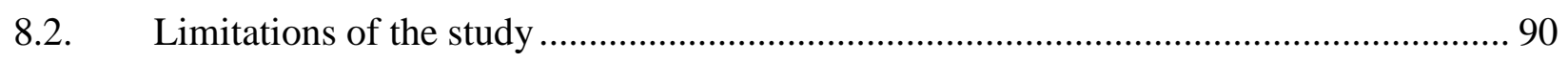

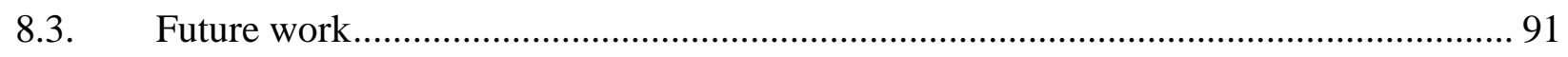

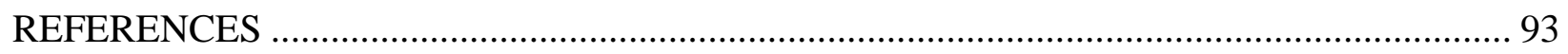




\section{LIST OF TABLES}

Table 1: Laminate stacking sequence of composite nail [84] ............................................. 52

Table 2: Material properties of CF/PA12, CF/EPOXY, polymeric core, CoCrMo, Ti6Al4V, 316L stainless steel, cortical and cancellous bones [9, 13, 16, 84]. L and T stand for longitudinal (fiber

direction) and transverse (normal to fiber direction) directions in the lamina, respectively....... 54 


\section{LIST OF FIGURES}

Fig. 2.1: Bone remodeling as described in strain energy density model [43] ........................... 8

Fig. 2.2: Schematic representation of bone as an open thermodynamic system [8] ................... 8

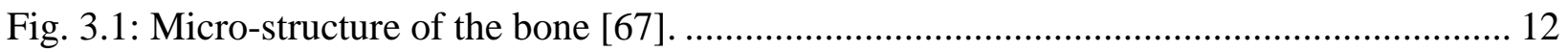

Fig. 3.2: Structure of cancellous bone in the distal end of the femur [69]................................ 13

Fig. 3.3: Schematic representation of bone remodeling process [43] ................................... 14

Fig. 3.4: Anatomy of the human lower limb[72] .............................................................. 15

Fig. 3.5: Four perspectives of the human femur [71] ..................................................... 16

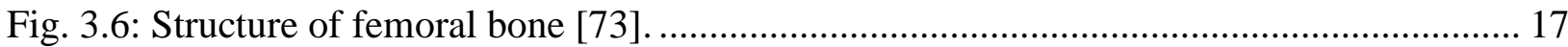

Fig. 3.7: Four types of proximal femur fractures: (a) subcapital neck fracture, (b) transcervical neck fracture, (c) basicervical neck fracture, (d) intertrochanteric fracture, (e) subtrochanteric

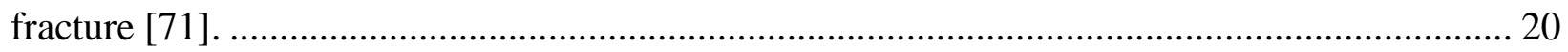

Fig. 3.8: Five types of forces that may fracture femoral shaft: (a) tensile force, (b) compressive force, (c) torsional force, (d) bending force, (e) combination of bending and compressive forces [71].

Fig. 3.9: Four types of distal femur fractures: (a) supracondylar fracture, (b) intercondylar fracture, (c) condylar fracture, (d) comminuted fracture extending into shaft [71] .............................. 22

Fig. 3.10: Hip joint: (a) location of hip joint in coronal view, (b) detailed view [72]............... 24

Fig. 3.11: A diseased hip joint affected by osteoarthritis [43] .......................................... 25

Fig. 3.12: A diseased hip joint affected by rheumatoid arthritis [43] .................................. 25

Fig. 3.13: A diseased hip joint affected by avascular necrosis [43] . ..................................... 26

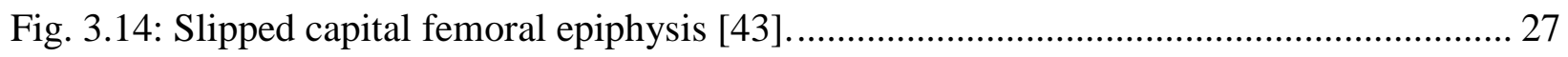

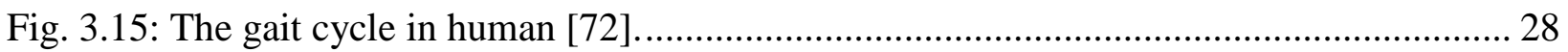

Fig. 3.16: Hip joint reaction force during the gait cycle [80]. ............................................... 29 
Fig. 4.1: External fixation: (a) illustration demonstrating external fixator, (b) X-ray picture

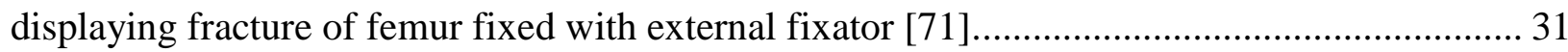

Fig. 4.2: Intramedullary nailing: (a) illustration demonstrating completed implant, (b) X- ray

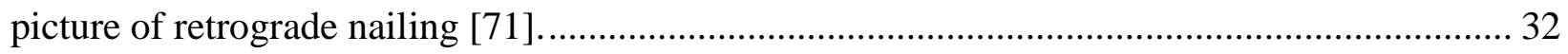

Fig. 4.3: Neutralization plate secured with regular bone screws along with two lag screws [81]. 35

Fig. 4.4: Buttress plate with wide surface area near to the distal epiphysis [81] ...................... 35

Fig. 4.5: Compression plate secured with bone screws and pulled by tensioner [81] ............... 36

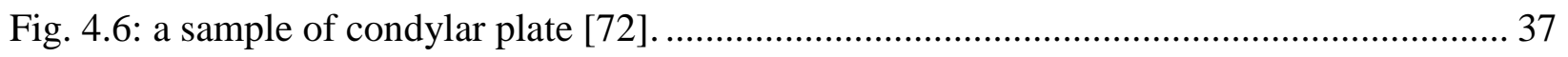

Fig. 4.7: The components of hip joint prosthesis [43] .................................................... 38

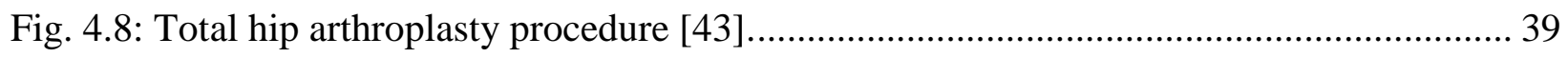

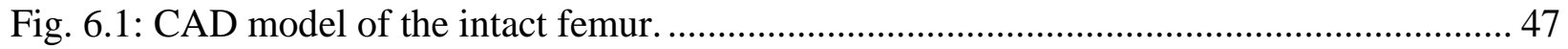

Fig. 6.2: CAD model of: (a) conventional hip implant (Stryker Exeter), (b) unconventional hip implant, (c) bone fracture plate (8-hole Zimmer) with screws, (d) IM nail (Stryker T2 nail)..... 48

Fig. 6.3: CAD model of: (a) femoral shaft, (b) femoral head and neck, (c) conventional hip implant.

Fig. 6.4: CAD model of unconventional hip implanted femur: (a) posterior view, (b) medial view. 50

Fig. 6.5: CAD model of hip implanted femur with (a) lateral plate, (b) anterior plate. 51

Fig. 6.6: CAD model of: (a) the femur with the intramedullary nail inside, (b) cortical bone is removed for better visualization. 52

Fig. 6.7: Boundary conditions shown on the typical intact femur. ....................................... 55

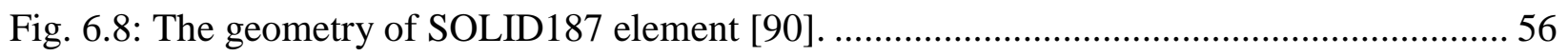

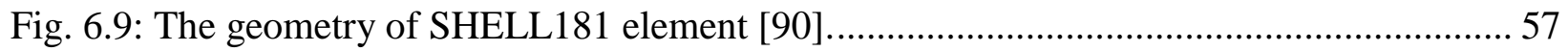

Fig. 6.10: The geometry of CONTA174 element [90]................................................... 58

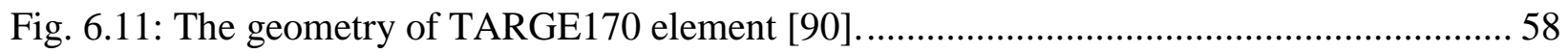


Fig. 6.12: Typical results of mesh sensitivity analysis for the intact femur.

Fig. 6.13: Typical meshed constructions: (a) intact, (b) hip implanted (with conventional implant), (c) lateral plated, (d) anterior plated and (e) IM nailed femurs. 60

Fig. 6.14: Iterative process of the thermodynamic-based model (mechano-biochemical model) for bone remodeling simulation. 62

Fig. 7.1: Convergence of bone remodeling simulations. 64

Fig. 7.2: Density distribution of the intact femur $\left(\mathrm{g} / \mathrm{cm}^{3}\right)$ : (a) anterior view, (b) posterior view. 65

Fig. 7.3: Density distribution of the intact femur in the coronal section: obtained by (a) mechanobiochemical model, (b) X-ray (Reprinted with permission, from [97]). 66

Fig. 7.4: Post-operative bone density distribution in response to conventional hip implant $\left(\mathrm{g} / \mathrm{cm}^{3}\right)$ : (a) anterior view, (b) posterior view, (c) coronal section of posterior view. 67

Fig. 7.5: Graph showing the post-operative bone density vs. number of iteration for zone 7...... 68

Fig. 7.6: Percent change in bone density due to the long-term presence of conventional hip implant: (a) posterior view, (b) three transverse segments, (c) medial view. 69

Fig. 7.7: Percentage of bone loss/formation in Gruen zones due to the long-term presence of conventional hip implant. 71

Fig. 7.8: Periprosthetic bone loss observed by [25] (Black values) compared to with that obtained by the mechano-biochemical model (Red Italic values). 72

Fig. 7.9: Post-operative bone density distribution in response to (a) CoCrMo, (b) Ti6Al4V, (c) $\mathrm{CF} / \mathrm{PA} 12$ unconventional hip implants. 74

Fig. 7.10: Percent change in bone density due to the long-term presence of (a) CoCrMo, (b) Ti6A14V, (c) CF/PA12 unconventional hip implants. Cutting plane line in all three constructions passes through the proximal metaphysis. 75

Fig. 7.11: Percentage of bone loss in Gruen zones due to the long-term presence of unconventional hip implants. 77

Fig. 7.12: Post-operative bone density distribution in response to hip implant and fracture plate: (a) lateral plated femur, (b) anterior plated femur. 80

Fig. 7.13: Percent change in bone density due to the long-term presence of the hip implant and fracture plate: (a) lateral plated femur, (b) anterior plated femur. 81 
Fig. 7.14: (a) femoral shaft six zones used to compare bone loss in the regions beneath the plate, (b) percentage of bone loss in six zones due to the long-term presence of hip implant and fracture plate. 82

Fig. 7.15: (a) Selected zone between the second and third hole from the distal end of the plate, (b) bone loss in four quadrants of the selected zone. 84

Fig. 7.16: Percentage of bone loss (intact vs. nailed femur) due to the long-term presence of IM nail for eight configurations. 85

Fig. 7.17: Post-operative bone density distribution in response to (a) C5, (b) Ti6Al4V nails.... 85

Fig. 7.18: Percent change in bone density due to the long-term presence of (a) C5 nail, (b) Ti6Al4V nail. 86

Fig. 7.19: (a) Selected four zones in the femoral shaft, (b) percentage of bone loss in six zones due to the long-term presence of IM nails. 87 


\section{NOMENCLATURE}

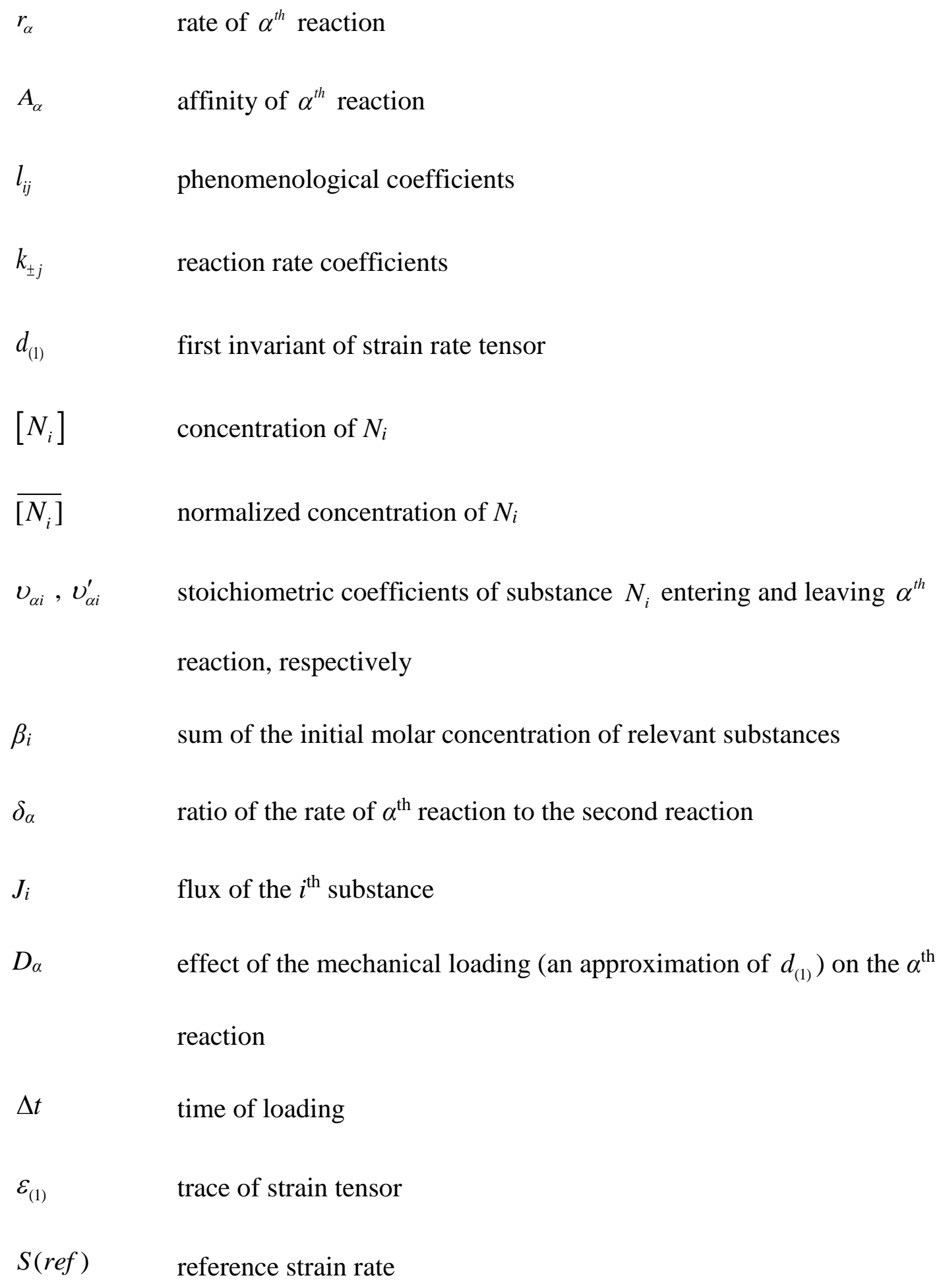




$\begin{array}{ll}D_{\alpha}(\text { ref }) & \text { influence of the reference strain rate on the } \alpha^{\text {th }} \text { reaction } \\ \rho & \text { bone density } \\ \rho_{0} & \text { initial bone density } \\ E_{\mathrm{Old}_{-} B} & \text { modulus of elasticity of old bone } \\ E_{\mathrm{New}_{-} B} & \text { modulus of elasticity of new bone }\end{array}$




\section{CHAPTER 1 : INTRODUCTION}

\subsection{Motivation}

Artificial joint prostheses and fracture fixation devices are implanted in patients with diseased or damaged joints or fractured bones to lessen pain and restore the appropriate function. After about 10 years of service, some serious complications such as bone infection and implant failure could happen which are mainly linked to the implant excessive micro-motion, the production of wear particles at the articular joint, and stress shielding at bone-implant interface. Metallic implants including hip implants and fracture fixation devices are much stiffer than bone, and thus carry a high amount of load compared to bone. Consequently, due to this abnormal load sharing, the bone around the implant is stress shielded which leads to bone loss, painful implant loosening and failure eventually. Therefore, considerable attention must be paid to such clinical cases through medical examinations such as dual energy X-ray absorptiometry (DEXA). However, it is not always possible to follow-up the long-term behaviour of the bone around the implants as follow up research is expensive and time consuming. Thus, it is important to develop and use mathematical models to predict bone remodeling and monitor the evolution of bone density after implantations to identify the risk of periprosthetic bone loss and improve the overall design of implants. To this end, many models have been developed, so far [1-5]. These models mainly fail to take into account the combined effect of metabolic factors and mechanical stimulus in bone remodeling process [4]. However, in the current study, a mechano-biochemical bone remodeling model (thermodynamicbased model developed by [6] and modified by [7, 8]) was employed which links the mechanical factors to biological ones, to more realistically predict bone density changes in response to orthopedic implantations. 


\subsection{Thesis objectives}

The main objective of this research study is to adapt and use a previously developed mechanobiochemical model (thermodynamic-based model developed by [6] and modified by $[7,8]$ ) to predict bone density evolution before and after orthopedic implantations. Therefore, to accomplish the research study and achieve the main objective, the following sub-objectives were set out:

1) Predicting bone remodeling in response to conventional metallic hip implant (CoCrMo) with the geometry of Stryker Exeter hip stem: size 2, offset $37.5 \mathrm{~mm}$.

2) Predicting bone remodeling in response to unconventional metallic (CoCrMo and Ti6Al4V) and composite (CF/PA12) hip implants developed by [9].

3) Predicting bone remodeling in response to hip implant ( $\mathrm{CoCrMo})$ and anterior or lateral plate (316L surgical-grade stainless steel) with the geometry of 8-hole Zimmer fixation plate, length: $246 \mathrm{~mm}$.

4) Predicting bone remodeling in response to metallic (Ti6Al4V) and composite (CF/Epoxy) intramedullary (IM) nails with the geometry of Stryker T2 femoral nail, length: $420 \mathrm{~mm}$.

The research results can assist in improving the design of implants and monitor the risk of bone loss after orthopedic surgeries. Having an understanding of the long-term behaviour of implanted bones can help clinicians choose proper surgical techniques, and help bioengineers design more effective orthopedic implants. 


\subsection{Thesis outline}

The thesis consists of eight main chapters. Chapter 1 provides the motivation behind the research, its importance and the objectives set out to accomplish. Chapter 2 focuses on literature review of bone remodeling mathematical models. It explains the existing bone remodeling models and illuminates their pros and cons. Also, it introduces early works which investigated bone response to different orthopedic implantations. Chapter 3 provides the background knowledge about human anatomy including femur, hip joint, gait cycle and bone remodeling mechanisms. Since this research study focuses on bone response to orthopedic surgeries, the author provided readers with the background knowledge regarding different orthopedic implantations including THA, plating and IM nailing via chapter 4 . Chapter 5 explains the thermodynamic-based model and its formulation process including modifying the law of mass action in order to incorporate the mechano-biochemical coupling into the model. It also introduces the power law relation between the bone density and elastic modulus [10] which was employed in the current study. Chapter 6 provides readers with the development procedure of computer aided drafting (CAD) models, the details of finite element modeling including element types, material properties of the implants, boundary conditions, the algorithm of thermodynamic-based model, etc. In chapter 7, the results of the research study are presented, discussed and compared to the previous studies in the literature.

Finally, in chapter 8 , the thesis ends up with concluding the major findings of the study, introducing the limitations and suggesting paths for future work. 


\section{CHAPTER 2 : LITRATURE REVIEW}

\subsection{Mathematical models}

Periprosthetic bone loss following orthopedic implantations is one of the most serious concerns compromising patient's life quality and is believed to be the result of stress shielding and osteolysis induced by the presence of wear debris [11, 12]. Stress shielding caused by improper load sharing between bone and implant is associated with bone resorption and occurs through bone remodeling $[4,13-16]$. The mechanism of bone remodeling was first postulated by Wolff's law stating that the reduction of mechanical stress causes bone to adapt itself by reducing its mass [17-20]. In the case of total hip arthroplasty, severe mass reduction may cause loosening of the implant which leads to the failure of hip replacement. Eventually, this situation requires a revision surgery, which is more complicated than primary THA and has less satisfactory outcomes [21, 22].

Periprosthetic bone loss is attributed to implant design $[23,24]$ and pre-operative bone quality [25]. Several experimental studies and computational models have been developed to understand the relationship between the mechanical loading and the functional adaptation of bone. Also, using dual energy x-ray absorptiometry (DEXA), many researchers have measured the bone mineral density (BMD) after implantation to investigate bone loss [25-28]. Since it is not always feasible to follow-up the long-term behaviour of the bone in response to implantation, it is important to develop realistic models to predict the bone evolution and thus monitor bone remodeling. To date, several bone remodeling theories $[1-5,18,29-34]$ have been developed which can be classified into three categories of mechanical, mechanobiological and biochemical models whose characteristics are as follows [13]. 
First, mechanical models predict bone adaptation based on mechanical stimuli, such as stress, strain and strain energy density, without considering biological factors $[4,30,35]$. In this category, the strain energy density based model (explained in section 2.1.1) is probably the most popular one which suggests that there is a range of strain (or strain energy density) values, so-called as the dead zone, where there is no bone remodeling. Strain (or strain energy density) below this range will cause bone resorption and strain (or strain energy density) above this range will initiate bone formation [4]. Another example of such models is developed by [30] which is based on cyclic energy dissipation as a measure of bone damage. According to this model, when damage is created during cyclic loading of the bone, a portion of the energy transferred during the loading phase is not recovered during the unloading phase. This energy is dissipated through some damage process such as the creation and extension of fracture surfaces. Therefore, the energy dissipated in a loading cycle can be used as a measure of the damage created during that cycle. In this model, the stimulus which initiates bone remodeling is formulated as proportional to the damage energy dissipation summed over all daily loading cycles. Although these models have been to some extent successful in predicting normal bone architecture, they have some major drawbacks, i.e. they use only mechanical signals to stimulate bone remodeling and fail to consider the underlying biological mechanisms that control bone remodeling.

Second, mechanobiological models aim at taking into effect biological as well as mechanical factors responsible for bone remodeling. These models were initially introduced via Frosts' mechanostat theory [1]. This theory states that bone adapts its strength to keep the strain caused by physiological loads close to a set-point. If strain level is below a set point, bone is resorbed, and if it exceeds this set point, new bone is formed. The mechanostat theory is a qualitative theory because the set-point is not specified. Several mechanobiological models have been presented so 
far $[3,36-38]$. However they are not complete for full understanding of the coupling between mechanical stimulus and bone biological response [2]. A model of this category [3] is based upon the separation of osteoblastic and osteoclastic activities. According to this model, osteoclasts are recruited and activated either where microcracks occur or in disused areas of the bone. The dynamic forces of daily living are known to produce microcracks. Mechanosensitive cells, called osteocytes, which derive from osteoblasts, sense a mechanical signal due to external load transfer through the architecture and locally recruit osteoblast to do the bone formation. The mechanical signal which is sensed by osteocytes is assumed to be the strain energy density.

Third, biochemical models are based on the activities of osteoblasts and osteoclasts to obtain an insight into the bone remodeling process at a cellular level. The first model of this kind described the differential activity of the Parathyroid Hormone (PTH) which acts as a regulator for bone resorption and formation [39, 40]. Another biochemical model [41] studied the role of hormones such as autocrine and paracrine in the regulation of bone remodeling while [42] proposed a signaling pathway known as RANK/RANKL/OPG to regulate bone cell activities. The most noticeable defect of these cell-based models is that they do not take into account the mechanical stimulus in the bone remodeling process.

Therefore, in order to improve the understanding of bone remodeling, it is crucial to include all factors (i.e., mechanical, biological and biochemical) concurrently. To do so, some relatively new models (Mechano-biochemical models $[6,8,13,33]$ ) were proposed in which the mechanical factors are linked to the chemical and biological ones. $[6,8,13]$ are based on irreversible thermodynamics in which bone is considered as an open self-organizing system capable of exchanging matter, energy and entropy with its surroundings. In the current study, the 
thermodynamic-based model (mechano-biochemical model) was used to develop a predictive tool for bone remodeling in response to orthopedic implantations (i.e. THA, plating and IM nailing).

\subsubsection{Strain energy density model}

Since in the most of mechanical based models, strain energy density is considered as the mechanical stimulus of bone remodeling mechanisms, it seems worthy to explain this model in detail. The strain energy $\left(U_{a}\right)$ which controls the rate of bone remodeling can be expressed in term of stresses $(\sigma)$ and strains $(\varepsilon)$ as follows [14].

$$
U=\frac{1}{2}\{\sigma\}\{\varepsilon\}
$$

In this model, the relation between the bone density change with time $(d \rho / d t)$ and strain energy defines the governing rule of bone remodeling process, stated as [14]:

$$
\begin{array}{lrl}
\frac{d \rho}{d t}=B\left(\frac{U_{a}}{\rho}-k(1+s)\right) & \frac{U_{a}}{\rho} \geq k(1+s) \\
\frac{d \rho}{d t}=B\left(\frac{U_{a}}{\rho}-k(1-s)\right) & \frac{U_{a}}{\rho} \leq k(1-s) \\
\frac{d \rho}{d t}=0 & k(1-s)<\frac{U_{a}}{\rho}<k(1+s)
\end{array}
$$

where $B$ is the slope of the curves shown in the Fig. 2.1 and $k$ is the signal value in the intact femur. As seen in Fig. 2.1, this model assumes that a certain threshold level (either in over-loading or under-loading) must be exceeded to trigger bone remodeling mechanisms. This range is known as dead zone, measuring $k(1 \pm s)$. When $\frac{U_{\mathrm{a}}}{\rho} \geq k(1+s)$, bone formation occurs, while bone resorption happens when $\frac{U_{\mathrm{a}}}{\rho} \leq k(1+s)$. 


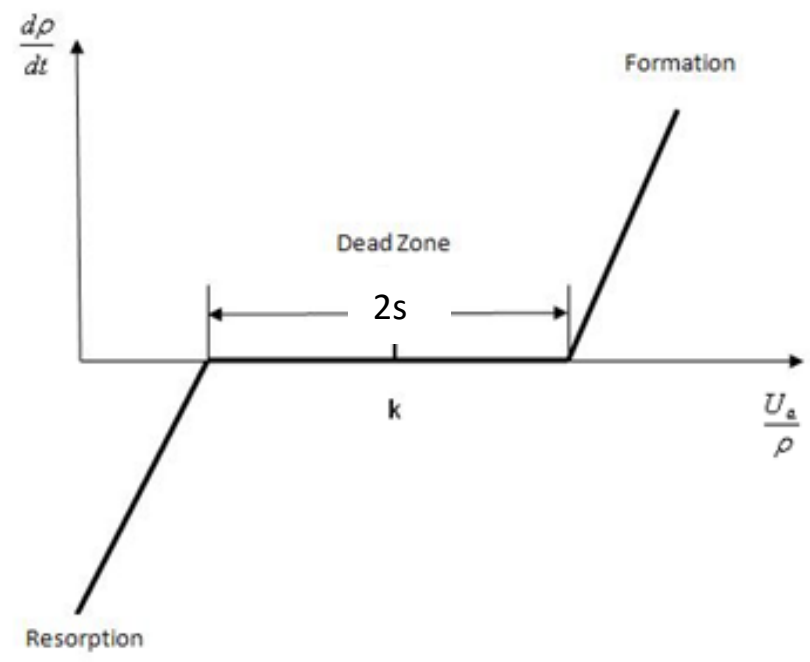

Fig. 2.1: Bone remodeling as described in strain energy density model [43].

\subsubsection{Thermodynamic-based model (Mechano-biochemical model)}

In the thermodynamic-based model (mechano-biochemical model) developed [6], bone resorption and formation phases, performed by osteoclasts and osteoblasts cells, are considered as the steps of bone remodeling mechanisms. Bone, as shown in Fig. 2.2, is hypothesized as a thermodynamic system exchanging matter, energy and entropy with its surroundings.

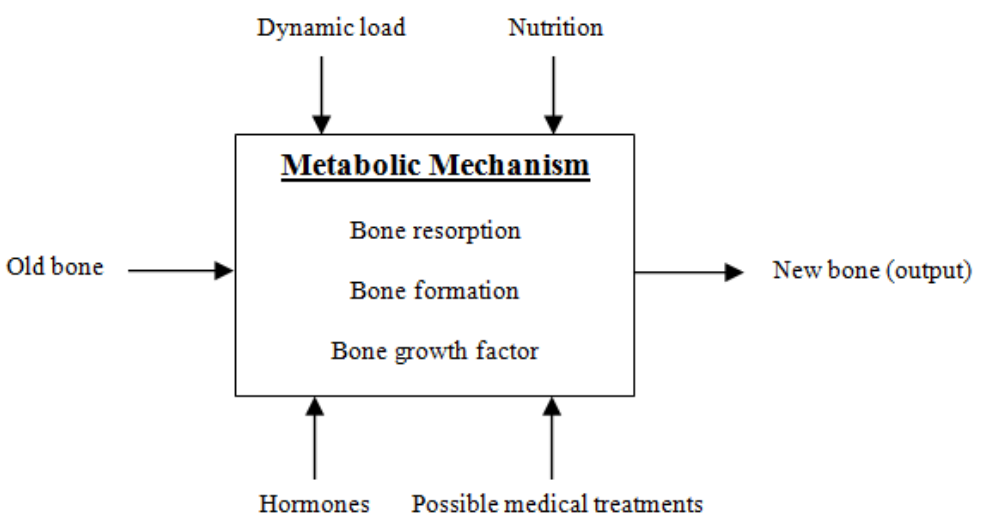

Fig. 2.2: Schematic representation of bone as an open thermodynamic system [8]. 
In the thermodynamic-based model, bone remodeling process is defined by a set of biochemical reactions which are in the form of Menten-Michaelis enzyme reaction (Eq. (2-3)) [44] and the modified version of the law of mass action $[6,8,13]$ is incorporated into the model to include the effect of mechanical loading on the biochemical reactions whose details are provided in chapter 5.

$$
E+S \underset{k_{-\alpha}}{\stackrel{k_{+\alpha}}{\rightleftarrows}} S E \longrightarrow E+P
$$

Enzyme $(E)$ retroactively binds to substrate $(S)$ to form the complex $(S E)$. Afterward, the complex breaks down into enzyme and product $(P)$.

\subsection{Early works on bone remodeling in response to composite implants}

A number of numerical, in vitro and in vivo studies reported that a more physiologic state following joint replacement could be achieved by lowering implant stiffness [45-49]. Several studies employed fiber-reinforced composites as an alternative material of choice capable of obtaining desired stiffness and strength in different directions [50, 87]. These implants showed improved load sharing characteristics and resulted in less deviation from the natural state of stress in the bone following implantation [50-56].

Fiber reinforced composite hip implants have been designed to address stress shielding concerns while maintaining enough strength and corrosion resistance in hip replacements [9, 57-59]. However, one may not be able to thoroughly investigate the stress shielding spanned by flexible composite implants without investigating periprosthetic bone remodeling. On the other hand, there seems to be no data regarding long term clinical follow-ups of patients who underwent composite hip replacements as they have not been widely used in humans. While few studies investigated the load distribution across the bone implanted with composite hip prosthesis $[9,58,60,61]$, only a 
handful of researchers predicted periprosthetic bone remodeling after such implantation [13, 62]. Therefore, using realistic numerical models to predict bone remodeling in the presence of composite hip implants is of interest. In the current study, the thermodynamic-based model (mechano-biochemical model) $[6,8,13]$ was used to predict long-term bone density distribution around an unconventional biomimetic hip stem made up of fiber reinforced polymer composites (i.e. carbon fiber polyamide 12: CF/PA12). The results were then compared to those obtained in femurs implanted with titanium alloy (Ti6Al4V) and cobalt-chrome-molybdenum (CoCrMo) implants.

Moreover, several studies have investigated the use of carbon fiber (CF) reinforced composites for bone fracture plates $[50,53]$. However, there are few who studied the use of composite materials for IM nails [56]. In particular, there is no study in the literature regarding the long term clinical follow-ups of patients who underwent composite IM nail. Therefore, it seems worthy to predict bone remodeling in the presence of composite nail using realistic numerical models. In the current work, the long-term density distribution of femoral bone implanted with a composite nail made up of carbon fiber-epoxy (CF/Epoxy) with seven different stacking sequences was investigated. The results were then compared to the femur implanted with Ti6Al4V nail.

\subsection{Early works on bone remodeling in response to hip implant and bone plate}

There are a number of clinical follow-ups which investigated BMD changes following total hip arthroplasty (THA) [25-27] or after plate removal in diaphyseal fractures [63-65]. The latter investigations on BMD changes after plate removal in un-implanted bones were done in the 1980s or early 1990s, when plating rather than intramedullary nailing was considered as the treatment technique for diaphyseal fractures. Although periprosthetic fractures with stable hip prosthesis are 
commonly treated with a bone plate, limited data is available regarding long term BMD changes in femur implanted with both hip prosthesis and bone plate as in the case of Vancouver B1 periprosthetic fractures which are quite common. As long-term follow-ups are usually costly and time consuming, using a realistic model to predict the post-union BMD changes in periprosthetic femoral fractures seems to be essential and could provide clinicians with long term response of such implantation. In this research, femoral density changes in response to a bone fracture plate (316L medical grade stainless steel) and a hip implant (CoCrMo) were investigated using the mechano-biochemical model. BMD changes were separately evaluated for lateral plating and anterior plating and compared thereafter. As plate removal is being limited to symptomatic patients [66], having an understating of long term behaviour of the plated bone can help clinicians to choose proper plating techniques and bioengineers to design more efficient bone plates. 


\section{CHAPTER 3 : HUMAN FEMUR AND HIP JOINT}

\subsection{Bone structure}

Bones, with the microstructure illustrated in Fig. 3.1, basically have the function of supporting the weight and protecting the vital organs of body, e.g. brain, lungs and heart. Furthermore, the bones of the skeleton provide the primary site of hematopoiesis, i.e. origin of blood cells. Bones also stores the $99 \%$ of body's calcium and a good portion of other ions such as potassium, phosphor, sodium and magnesium. Moreover, bone marrow in the medullary canal is responsible for formation and development of red blood cells which deliver oxygen to all the cells of the body [36].

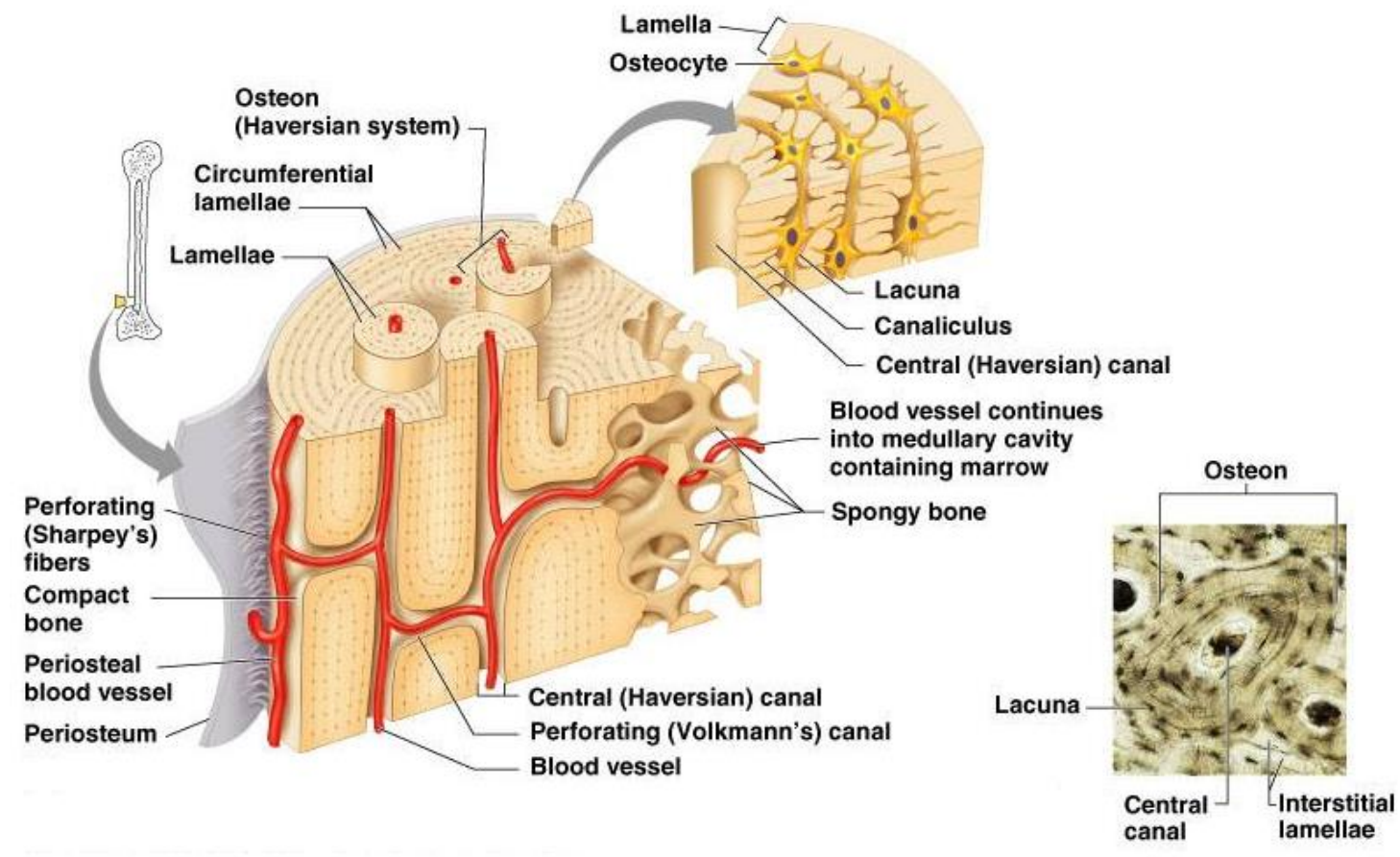

Fig. 3.1: Micro-structure of the bone [67]. 
There exist two types of bone tissue: cancellous and cortical bones. The cancellous bone is relatively softer, weaker, less dense and less stiff compared to the cortical bone which forms the structure of the bone. As shown in Fig. 3.2, the cancellous bone is a porous cellular solid with volume fraction of approximately $20 \%$. Its function is to distribute the load on outer more rigid part (cortical bone). Furthermore, it is able to absorb a lot of energy by way of self-deformation which is very essential during a fall, for instance [36].

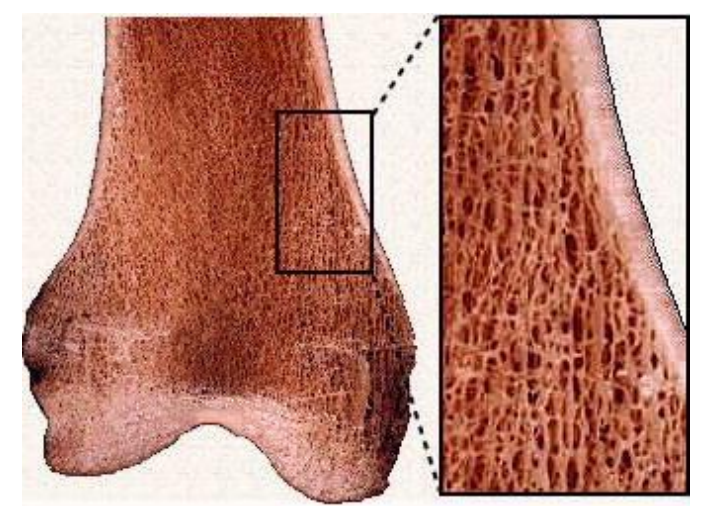

Fig. 3.2: Structure of cancellous bone in the distal end of the femur [69].

\subsection{Bone remodeling mechanisms}

Even after the longitudinal growth of the skeleton is completed, the bone retains its ability to adapt its internal structure by removing old bone and replacing it with newly formed bone; this process is called bone remodeling [39]. It is a fundamental property of living bone to adjust to a changing mechanical environment. The mechanism of bone remodeling was first postulated by Wolff in $19^{\text {th }}$ century. Wolff's law states that the reduction of mechanical stress causes bone to adapt itself by reducing its mass, either by getting thinner (external remodeling) or becoming more porous (internal remodeling) [20]. It is a complex process performed by coordinated activities of osteoclasts and osteoblasts. Osteoclasts are the cells responsible for resorbing mineralized bone. 
Osteoblasts are the cells capable of forming of the new bone matrix. The resorption and formation of bone in a healthy person are in balance to maintain skeletal strength and integrity. The process of bone remodeling is schematically shown in Fig. 3.3 [70]. In the first phase (resorption), osteoclasts attach to bone surface and break down the bone by eroding the matrix. Consequently, some small cavities are formed on the surface of the bone. Then, osteoblasts start forming new bone to fill the cavities. In the last phase, the bone surface is restored and covered by a layer of lining cells. The new bone is calcified and the bone remodeling process is completed.
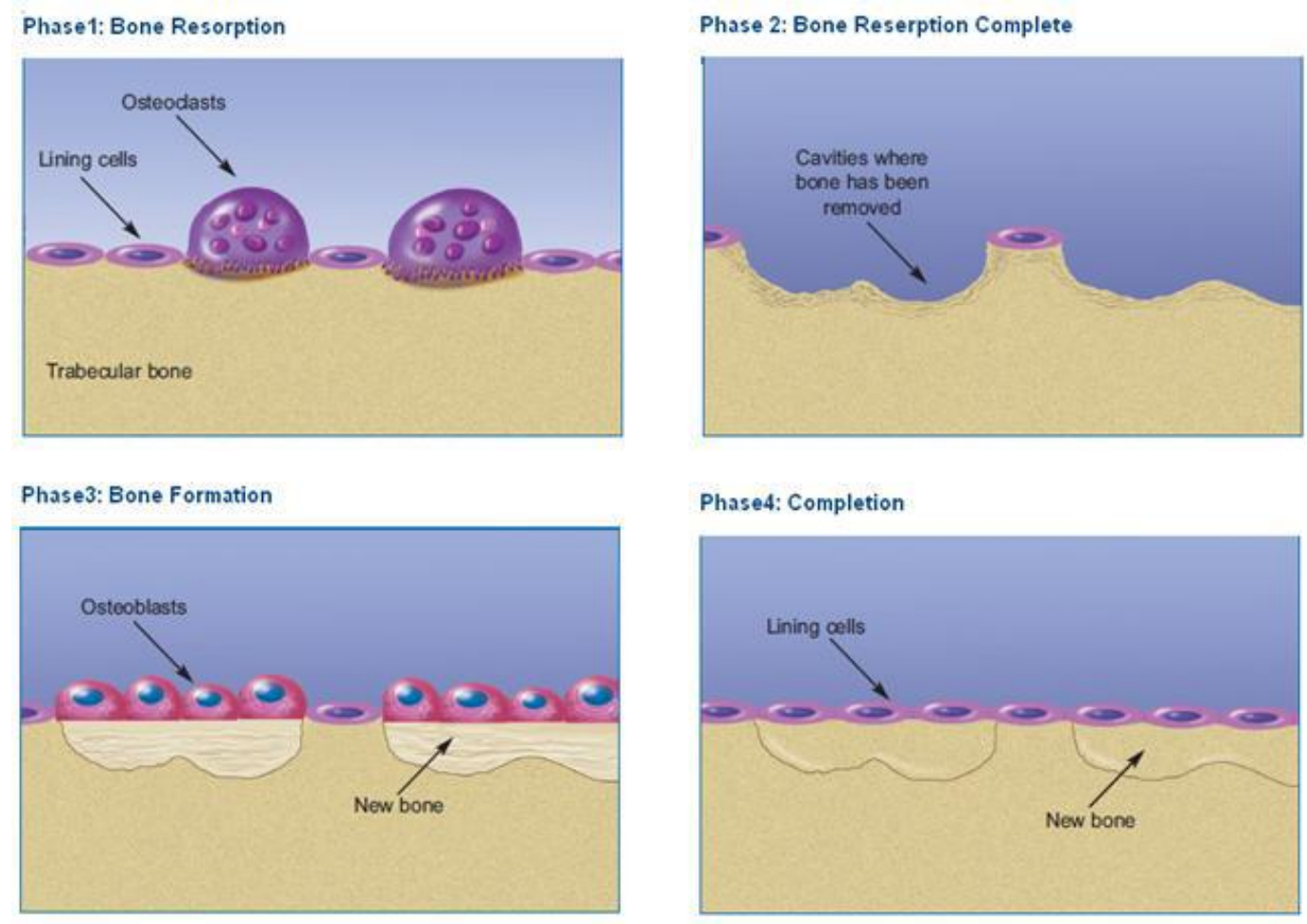

Fig. 3.3: Schematic representation of bone remodeling process [43].

According to Wolff's law, mechanical factors guide the bone adaptation mechanisms. If the mechanical stress acting on a bone decreases, the bone becomes less dense as there is no stimulus for continued remodeling. Conversely, the new bone is formed at those sites where mechanical strains are repeatedly detected. On the other hand, the Utah paradigm states that the biological 
mechanisms still need mechanical-free factors e.g. vitamins, hormones, etc. in order to function. The mechanical-free factors, in turn, can help or modulate the mechanical factors' guidance in bone remodeling process [40].

\subsection{Human femur}

As shown in Fig. 3.4, the most proximal bone in a human lower limb is the femur which is also referred to as the thigh bone. In healthy human, there exist two femurs which, with respect to the human coronal plane, are symmetrically placed inferior to the pelvis and superior to the fibula and tibia. The femur forms part of the hip joint on its proximal end and constitute the knee joint at the distal end. It represents the largest and longest bone in the human body and experiences loads that are typically twice the weight of an adult body. In the average adult male, a femur measures approximately $480 \mathrm{~mm}$ and $23.4 \mathrm{~mm}$, in length and diameter, respectively [71].

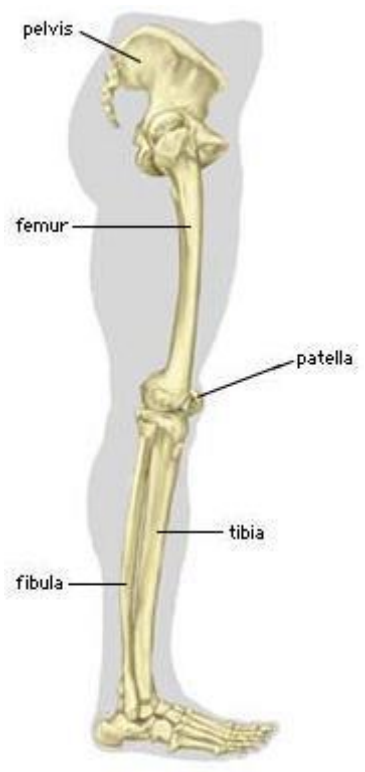

Fig. 3.4: Anatomy of the human lower limb [72]. 


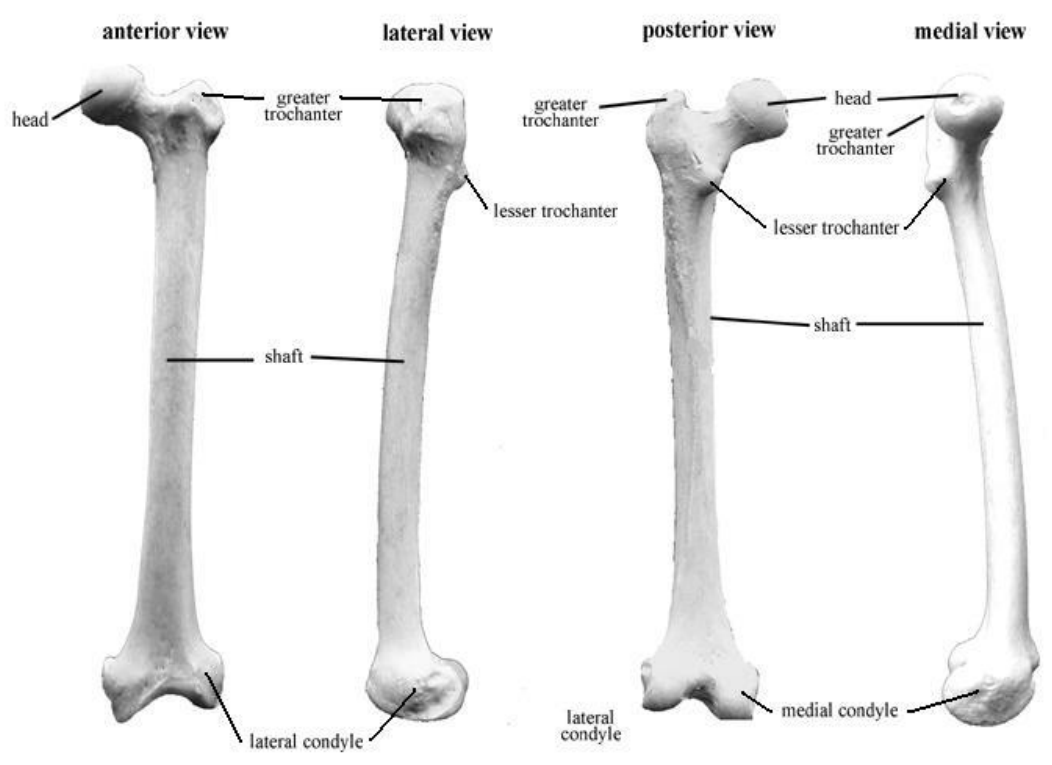

Fig. 3.5: Four perspectives of the human femur [71].

As depicted in Fig. 3.5, the femur primarily consists of a shaft and five protuberances including the medial condyle, lateral condyle, head, greater trochanter, and lesser trochanter [66].

The medial condyle and the lateral condyle are both positioned in the femur distal end, close to the knee joint. The greater trochanter is positioned laterally from a coronal view, while the head is positioned medially, both being located proximally. The lesser trochanter protrudes posteriorly, and is inferior to both the greater trochanter and the head. The protuberances associated with the femur distal and proximal ends are referred to as epiphyses which are comprised of sponge bones in the interior of the femur. The femoral shaft, which is so called diaphysis, is made up of the cortical bone, and hosts the medullary cavity which is associated with an interior empty space. As depicted in Fig. 3.6, metaphysis lies between diaphysis and epiphysis in both proximal and distal ends [71]. 


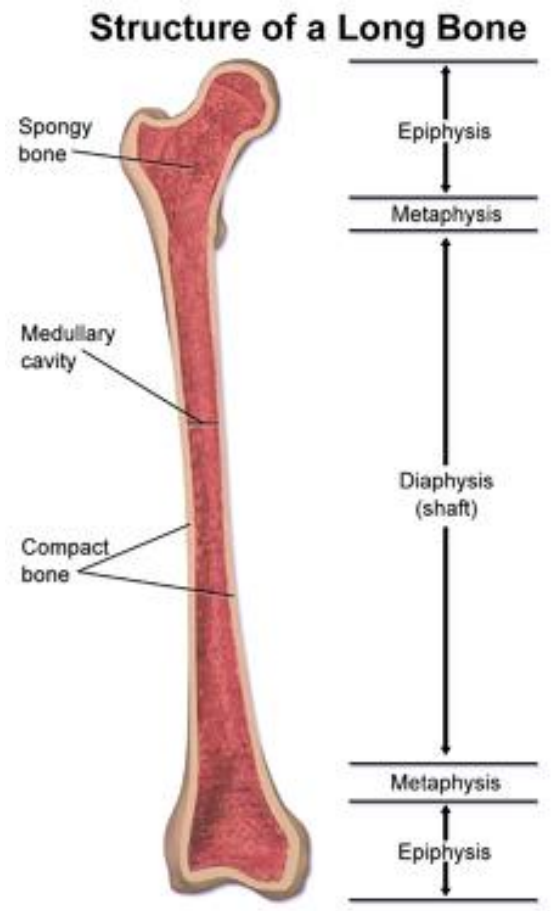

Fig. 3.6: Structure of femoral bone [73].

\subsection{Femur fracture and disease}

Femur fractures often result from either low-energy falls in patients suffering from osteoporosis or high-energy traumas (impact). Since human femur represents the strongest bone in the body, it could withstand great compressive loads prior to failure. Patients who have a fractured femur require immediate medical attention because the traumatic incident is generally linked to life threatening injuries. An injury to the femur may in turn disrupt blood supply via the quadriceps muscle. Disruption of blood supply may also imply bruising and extensive loss of blood [71].

\subsubsection{Osteoporosis}

In patients suffering from osteoporosis, the bone mineral density decreases to such a critical level that the bone microstructure is disrupted. Therefore, the risk of occurrence of bone fractures in such patients is much higher than that in healthy persons. Several major causes of osteoporosis 
have been identified including aging, improper nutrition and lifestyle, heredity, medications and illnesses $[66,72]$.

\section{- Aging}

Up to 28 million Americans suffer from osteoporosis which causes about 1.5 million fractures annually as reported by the American Academy of Orthopaedic Surgeons (AAOS). The institute further estimated that for individuals older than 65 years, one of five men and one in two women are at risk of fracturing a bone due to osteoporosis [72].

\section{- Improper nutrition and lifestyle}

Minerals such as calcium, magnesium, zinc, and phosphorus play a key role in the constitution of human bone. The lack of proper nutrition and low level of critical minerals in human bone may contribute to a decrease in bone mineral density, which may result in osteoporosis at any age. Improper lifestyle such as smoking, excess alcohol consumption, being underweight, and too much physical activity may also increase the risk of development of osteoporosis $[66,72]$.

\section{- $\quad$ Heredity}

Many studies believe that the existence of young people afflicted with osteoporosis can be explained by the fact that the race and family history of osteoporosis may increase the risk of developing osteoporosis. More specifically, an individual with Asian ancestry who characterized with a small and slender body build and fair skin faces greater risk of osteoporosis development $[66,72,74]$. 


\section{- Medications and other illnesses}

Specific medication programs such as steroids or illnesses such as thyroid complications have been associated with increased risk of osteoporosis as these cases may increase the osteoclastic activities [72].

\subsubsection{Physical impact}

A femur fracture may also result from physical impact. Taking a hit in the course of sporting activities or falling from a great height may lead in a fracture at any age. Femur fractures in children, however, more often, are attributed to physical abuse. Fractures to the femur manifest various relatively obvious symptoms, including severe pain, swelling, inability to move the leg, and deformity. In addition, a fracture to the femur may make the injured thigh shorter than the intact one, as a result of strong thigh muscles pushing broken femur edges out of alignment [66, $72,75]$.

\subsection{Femur fracture classification}

There are three broad categories of femur fractures including proximal femur fractures, distal femur fractures, and femoral shaft fractures. Each is described in detail below.

\subsubsection{Proximal femur fracture}

Proximal femur fractures are associated with the upper and bulbous portions of the femur, which is closest to the hip joint. This fracture type is likely to be accompanied by a hip joint failure and is more commonly experienced by the elderly or people suffering from osteoporosis. Proximal femur fractures, shown in Fig. 3.7, can be further categorised into three types, namely, intertrochanteric femur fracture, subtrochanteric femur fracture and femoral neck fracture [66, 71$]$. 


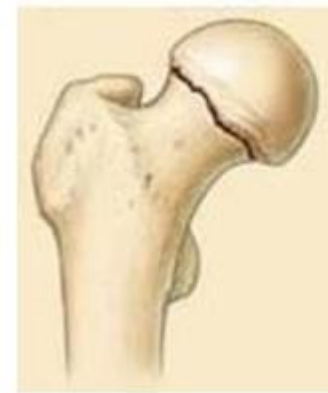

(a)

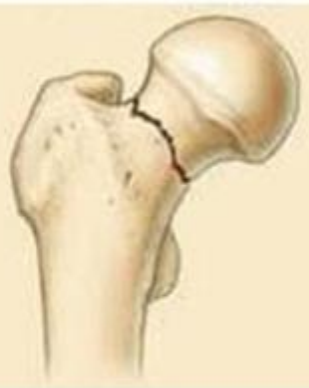

(b)

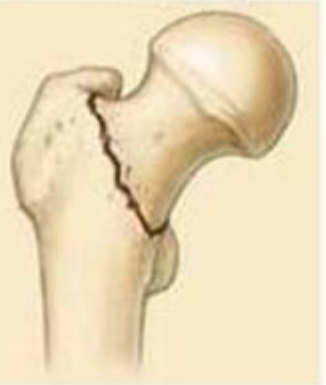

(c)

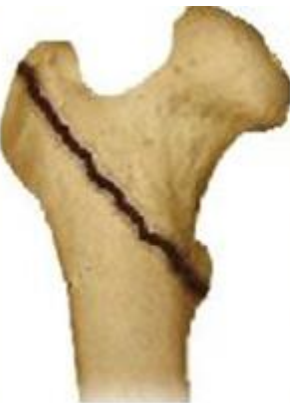

(d)

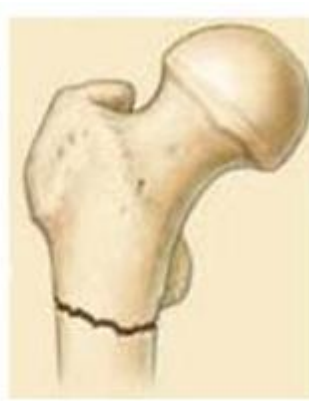

(e)

Fig. 3.7: Four types of proximal femur fractures: (a) subcapital neck fracture, (b) transcervical neck fracture, (c) basicervical neck fracture, (d) intertrochanteric fracture, (e) subtrochanteric fracture [71].

The intertrochanteric femur fracture occurs through the lesser and greater trochanter; while the subtrochanteric femur fracture line cuts just below the lesser trochanter. When the femoral head fractures off the femur, it leads to a femoral neck fracture. Subcapital, transcervical, and basicervical neck fractures are the three types of recognized femoral neck fractures [71].

\subsubsection{Femoral shaft fracture}

High-energy impacts and ill-fitting orthopedic implants which increase the stresses borne by the femur generally lead to femoral shaft fractures. Since the femoral shaft has a cylindrical structure, five classes of loads may lead to a fracture, including tension, compression, bending, torsion, as well as a combination of compression and bending as illustrated in Fig. 3.8 [75-77].

When a tensile force acts on the femoral shaft, the fractural pattern will be normal to the direction of the load, assuming that equal forces act on both sides. Such a fracture is referred to as a simple transverse fracture and occurs along the planes on which the tensile stresses are greatest. This fracture type does not generate small particles or bone debris $[72,75,77]$.

Great compressive loads could result in fractures along a plane that forms an oblique angle in relation to the direction of the load applied. Such loads apply great shear stresses on the oblique 
plane. This implies that the compressive forces lead to factures along the planes of high shear stresses $[71,77]$.

Conversely, high torsional forces lead to spiral type fractures. A small crack originating from the bone surface sets off a fracture that progress through the bone in a spiral fashion. The point of crack initiation occurs on a plane where the greatest shear stresses are experienced. The above pattern of fracturing is referred to as an oblique spiral fracture $[71,72,77]$.

Bending forces apply tension forces and compression forces on opposite halves of a femur, leading to a fracture pattern consistent with both tensile and compressive forces. Consequently, a simple transverse fracture will be witnessed on the femur side subjected to tension, and oblique fractures on the compression end. Two oblique fracture patterns are typically witnessed on the compression end, resulting in comminution. Such a fracture is referred to as a butterfly fracture, and the comminution is termed as a butterfly fragment $[72,77]$.

When a femoral shaft is exposed to a combination of compressive and bending forces, it reveals a fracture pattern similar to the one associated with application of bending forces only. However, in such loading circumstances, the butterfly fragment will assimilate more than $50 \%$ of the cortical bone. Consequently, there would be less than $50 \%$ cortical contact between the distal and proximal fragments. With increase in the compressive force applied, the number of pieces making up the butterfly fragment increases [72, 77]. 


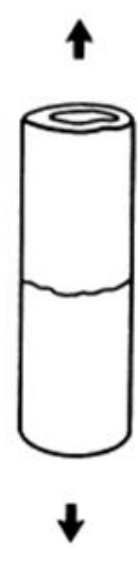

(a)

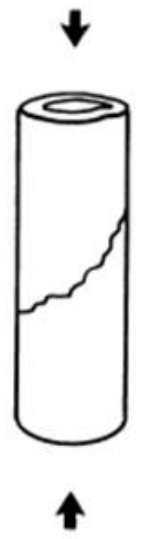

(b)

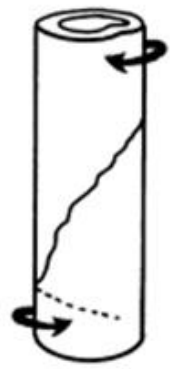

(c)

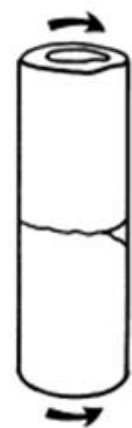

(d)

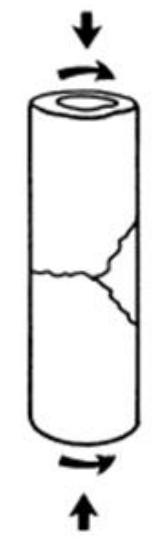

(e)

Fig. 3.8: Five types of forces that may fracture femoral shaft: (a) tensile force, (b) compressive force, (c) torsional force, (d) bending force, (e) combination of bending and compressive forces [71].

\subsubsection{Distal femur fracture}

Distal femur fractures are often associated with the $15 \mathrm{~cm}$ distal portion of the femur. This type of fracture may be witnesses in patients who have ill-fitting total knee prostheses. As shown in Fig. 3.9, there are four main categories of distal femur fractures, including supracondylar fracture, condylar fracture, intercondylar fracture, and comminuted distal femur fracture [78, 79].

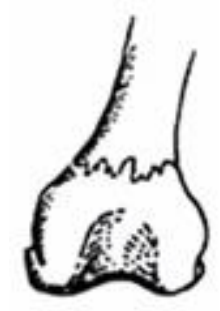

(a)

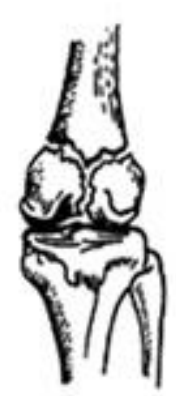

(b)

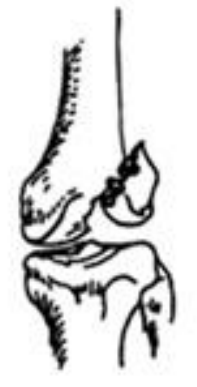

(c)

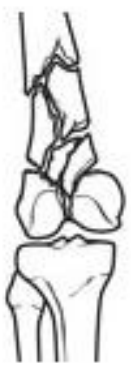

(d)

Fig. 3.9: Four types of distal femur fractures: (a) supracondylar fracture, (b) intercondylar fracture, (c) condylar fracture, (d) comminuted fracture extending into shaft [71]. 
Supracondylar fractures are found on the plane that is normal to the femur axis near the epiphysis edges, which is where the stresses are concentrated as a result of changes in the cross-sectional area. In a supracondylar fracture, the distal femur breaks off in a transverse direction from the rest of the femur.

Intercondylar fracture occurs between the medial condyle and the lateral condyle. This type of fracture splits the femur into three or more parts, depending on how severe the injury is.

Condylar fracture results in a femur with an oblique fracture pattern through one of the condyles while the other one is intact.

Severe physical impact may comminute the distal femur resulting in several segments with a fracture extending into femoral shaft. Treatment of this fracture type is complicated as alignment of femur and placing the fracture fragments in the proper positions are difficult $[72,78,79]$.

\subsection{Human hip joint}

In anatomy, the hip joint (acetabulofemoral joint) is located where the acetabulum of the pelvis and the head of the femur met as depicted in Fig. 3.10. The hip joint has two primary functions. Firstly, it has the role of supporting the weight of the body. Consequently, it facilitates static posture, for example, standing. Secondly, the acetabulofemoral joint facilitates the articulation and rotation of the head of the femur around the acetabulum, so the body is able to carry out dynamic motions, including walking and running [72]. 

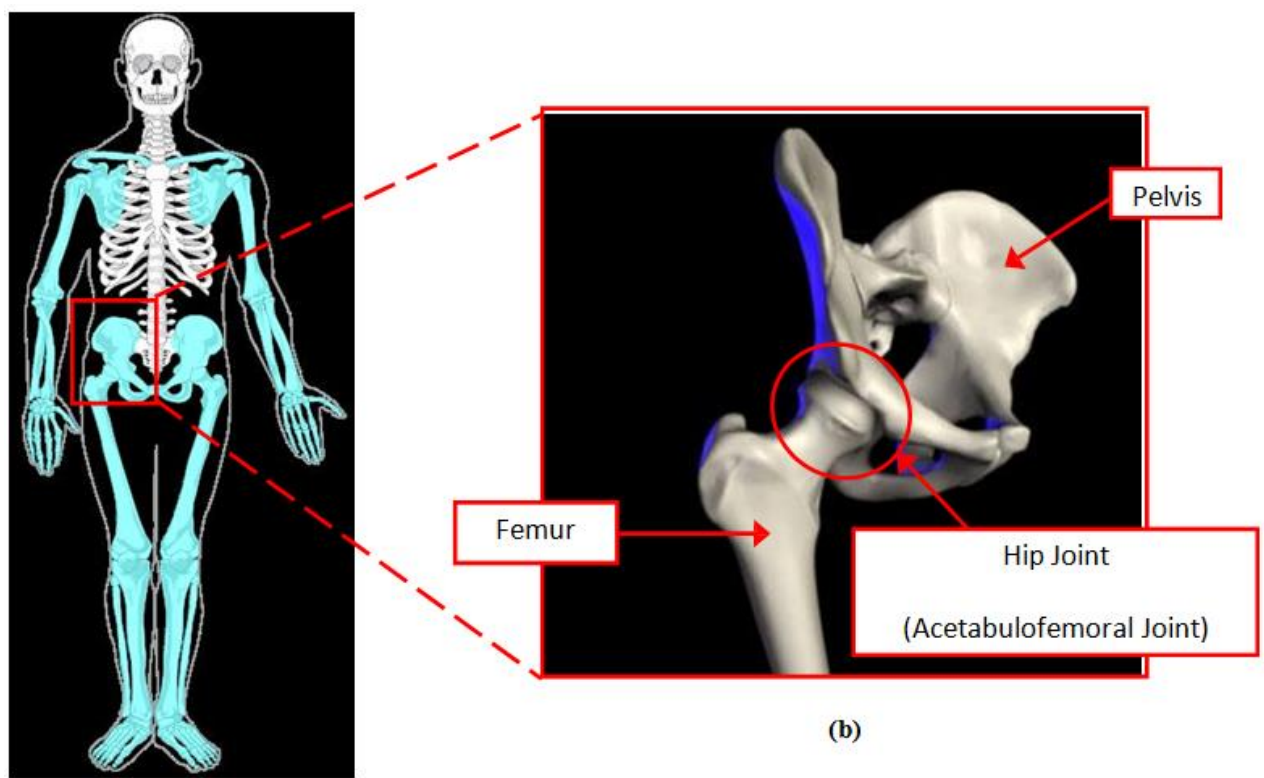

(b)

(a)

Fig. 3.10: Hip joint: (a) location of hip joint in coronal view, (b) detailed view [72].

\subsection{Hip joint fracture and disease}

Acetabulofemoral joint is the joint that bears the greatest load in the human body. For this reason, it is prone to a wide range of diseases that can lead to pain and dysfunction. Most of the diseases may be treated surgically through total hip arthroplasty (THA) [72]. The followings are several common diseases associated with the hip joint.

\subsubsection{Osteoarthritis}

Osteoarthritis is a degenerative disorder which deteriorates the hip joint at a relatively rapid rate, through breakdown of cartilage as shown in Fig. 3.11. This disease is the most common form of arthritis and afflicts persons aged over 60 or 70 years. Osteoarthritis is primarily caused by aging. As one ages, the protein makeup of cartilage breaks down while the water content in cartilage increases. In advanced cases, this protein degeneration process could result in loss of all the 
cartilage lining the bones in the joint sections. Loss of cartilage in turn causes additional friction between adjacent bones, leading to pain and restricted mobility [43].

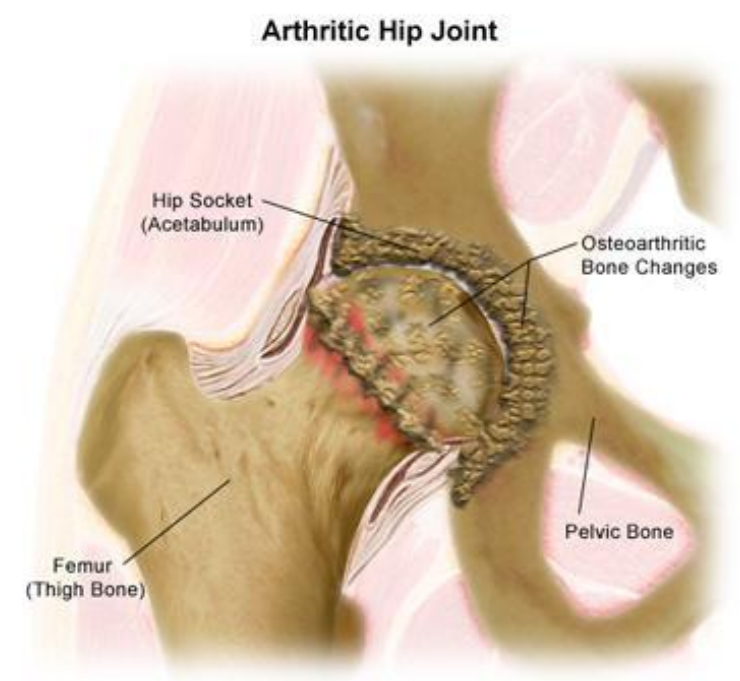

Fig. 3.11: A diseased hip joint affected by osteoarthritis [43].

\subsubsection{Rheumatoid arthritis}

Rheumatoid arthritis is a case where the immune system inadvertently launches attacks on healthy tissues. When the above occurs, it can lead to chronic inflammation in the joint.

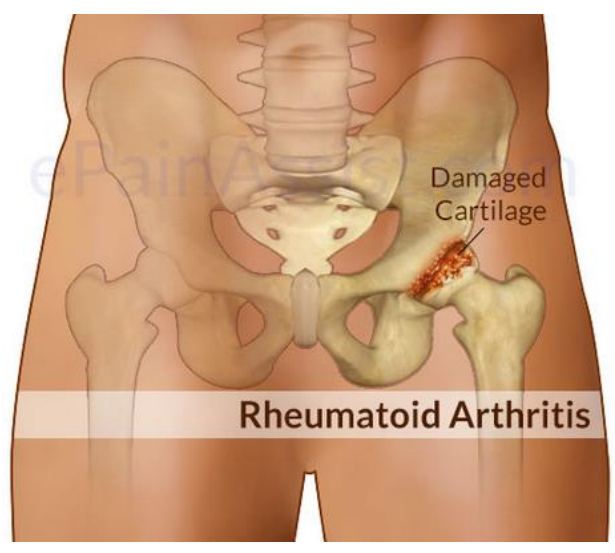

Fig. 3.12: A diseased hip joint affected by rheumatoid arthritis [43]. 
As shown in Fig. 3.12, the chronic inflammation in the joint could further lead to destruction of the cartilages which could lead to pain and deformities in the joint [43].

\subsubsection{Avascular necrosis}

Fig. 3.13 illustrates a hip joint afflicted by the avascular necrosis disease where the blood supply to the femoral head is inhibited or interrupted. This may cause to gradual bone death or even total collapse of the femoral head in severe cases [43].

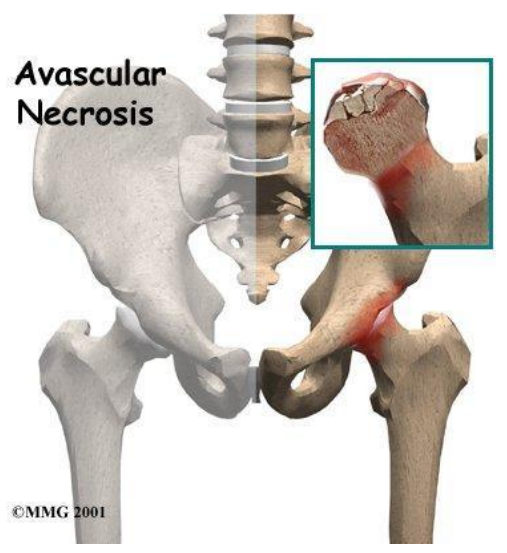

Fig. 3.13: A diseased hip joint affected by avascular necrosis [43].

\subsubsection{Slipped capital femoral epiphysis (SCFE)}

Slipped capital femoral epiphysis (SCFE) disorder occurs mostly in children with weak growth plates. In this disorder, the head of the femur slips off the femoral neck through the growth plate; it has three levels of severity:

- $\quad$ Mild (Position A in Fig. 3.14): a third of the femoral head has slipped off the femoral neck.

- Moderate (Position B in Fig. 3.14): a third to a half of the head of the femur has slipped off the femoral neck. 
- $\quad$ Severe (Position C in Fig. 3.14): more than half of the femoral head has slipped off the femoral neck [43].

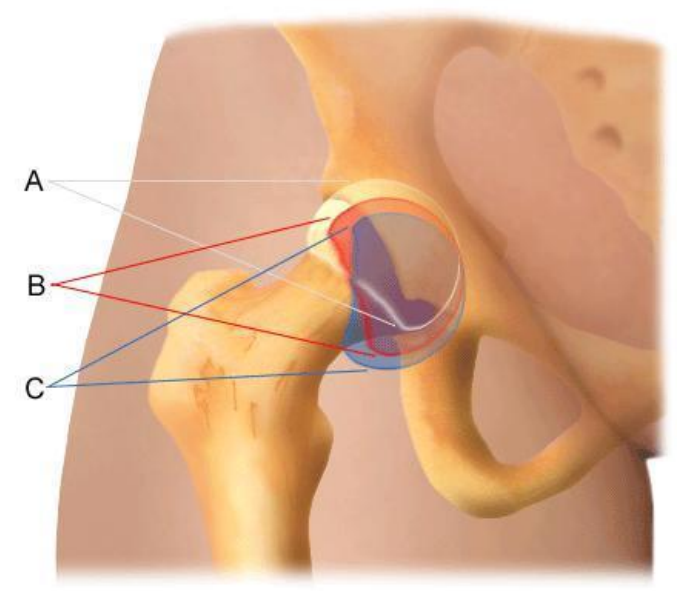

Fig. 3.14: Slipped capital femoral epiphysis [43].

\subsubsection{Physical impact}

Trauma encompasses any damage to the hip joint, which may be occasioned by a fall, workplace or athletic injuries, or a car accident. These events may damage the cartilage or other sections of the joint.

\subsection{The gait cycle and loading conditions}

Prior to investigating mechanical load applied on the femoral head, the gait cycle which describes the activity of walking in humans should be described. As illustrated in Fig. 3.15, the gait cycle is a time interval which starts when one foot contacts the ground until the same foot again touches the ground. The gait cycle consists of two phases; the stance phase (60\% of the gait) and the swing phase (40\% of the gait): 
The stance phase further divides into the loading response, mid-stance, terminal stance and preswing. The loading response starts when one foot contacts the ground and ends when the toe of other leg separates from the ground. This sub-phase is followed by the mid-stance which starts at the contra lateral toe-off and finishes when the centre of gravity of the body is directly placed over the reference foot. Then, the terminal stance starts and continues until the foot of the other leg comes into contact with the ground.

The swing phase further divides into initial swing, mid-swing and terminal swing. The initial swing begins at toe-off and ends when the reference knee arrives at the maximum flexion of 60 degrees. Afterward, the sub-phase of mid-swing starts and continues until the knee is perpendicular to the ground. At this moment, the terminal swing begins and continues until initial contact [43].

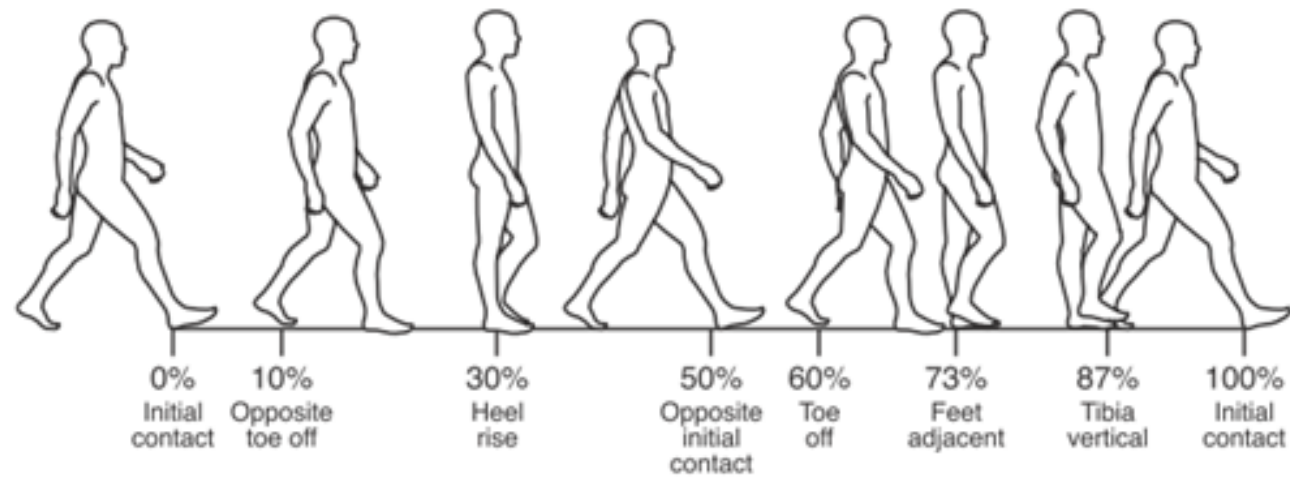

\begin{tabular}{|c|c|c|c|c|c|c|}
\hline $\begin{array}{c}\text { Laading } \\
\text { response }\end{array}$ & $\begin{array}{c}\text { Mid } \\
\text { stance }\end{array}$ & $\begin{array}{c}\text { Terminal } \\
\text { stance }\end{array}$ & $\begin{array}{c}\text { Pre } \\
\text { swing }\end{array}$ & $\begin{array}{c}\text { Initial } \\
\text { swing }\end{array}$ & $\begin{array}{c}\text { Mid } \\
\text { swing }\end{array}$ & $\begin{array}{c}\text { Terminal } \\
\text { swing }\end{array}$ \\
\hline & Right stance phase $=60 \%$ & Right swing phase $=40 \%$ \\
\hline
\end{tabular}

Fig. 3.15: The gait cycle in human [72].

As shown in Fig. 3.16, during the gait cycle, the hip joint is under the dynamic loading having two peak forces. The peak forces happen during the stance phase and may reach about seven times of the body weight in men. The magnitude of the force approximately remains at a same level equal 
to the body weight during the swing phase. The pattern of loading is roughly the same for men and women but there are significant differences in their magnitudes; as illustrated in Fig. 3.16, the maximum peak force during women's gait cycle limits to only four times the body weight. This quantitative discrepancy can be justified by differences between the pelvis width of males and females, differences in foot wear or in the inclination of neck-shaft angle [43].
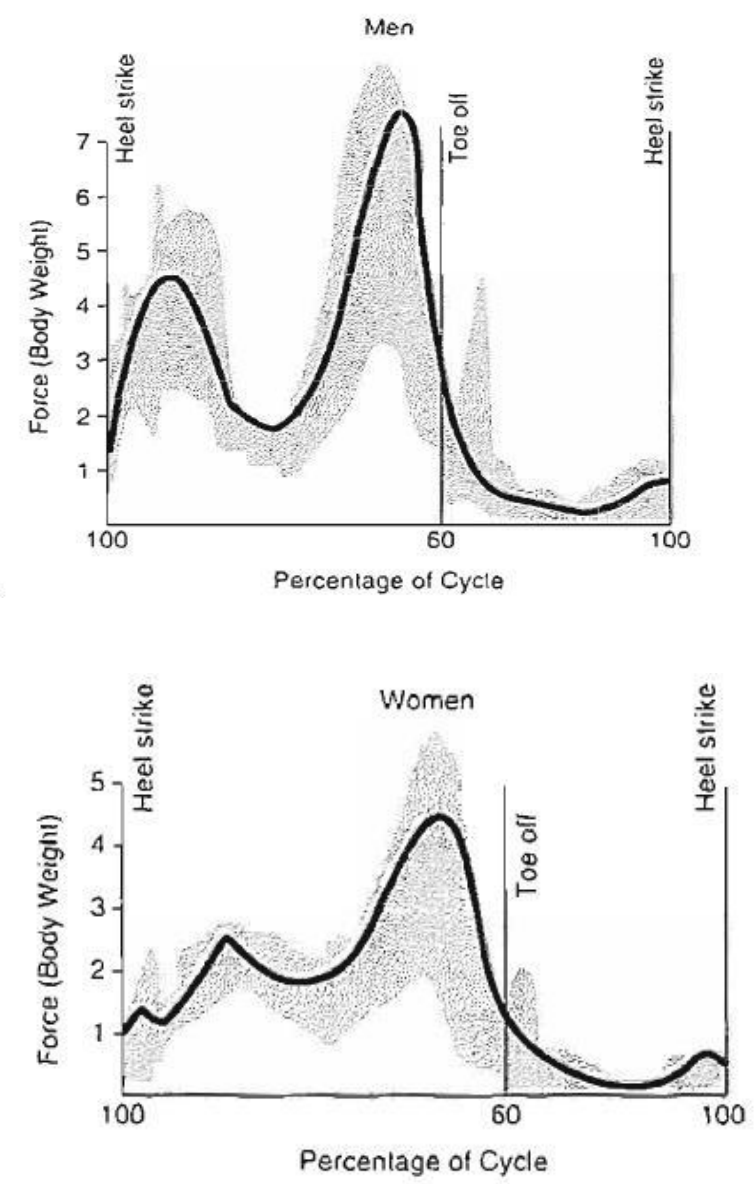

Fig. 3.16: Hip joint reaction force during the gait cycle [80]. 


\section{CHAPTER 4 : ORTHOPEDIC IMPLANTATIONS}

Numerous surgical fixation techniques are available with the aim of offering reliable and secure ways of healing fractured femurs. The most popular techniques include external fixation, plating, and intramedullary nailing. Often, intramedullary fixation and plating are preferred, unless the patient is non-ambulatory, or could have a serious complication in the course of an operation [78].

The human hip joint is subjected to a high amount of load, therefore is prone to severe damages and diseases. Most of the diseases may be treated surgically through total hip arthroplasty (THA) which a prosthesis implanted in the body replaces the damaged hip joint [66].

\subsection{External fixation}

External fixation represents a surgical technique conducted by placing pins through the lateral thigh muscles to put the femur fractured segments in their proper positions, as illustrated in Fig. 4.1. In doing so, the femur segments may be compressed without losing their natural dimensions, and become aligned properly. The fixator consists of a lateral ring-shaped frame to adjust the pin locations. This technique stabilizes the fractured femur without damaging the exterior wound any further. Moreover, this is an option for patients who have soft tissue damage or contamination with the femur fracture. This option is also applied when serious complications would occur if an operation were performed $[72,77,78]$.

External fixation presents several disadvantages. Firstly, it requires additional procedures following improvement of the patient's condition, since it alone does not guarantee a full recovery. Secondly, most of patients acquire infections around the sites where pins are inserted. Consequently, pins have to be meticulously prepared before the operation, while preventing 
infection and using appropriate antibiotics. Another disadvantage of external fixation is that the restriction of muscles could also restrain knee motions. Consequently, regular physical activities may not be undertaken while the external fixations are in use and patients have to be cautious of their motion because the rigid pins which are inserted between muscles, neurovascular systems, and tendons could rapture under excessive loading conditions [77, 78].

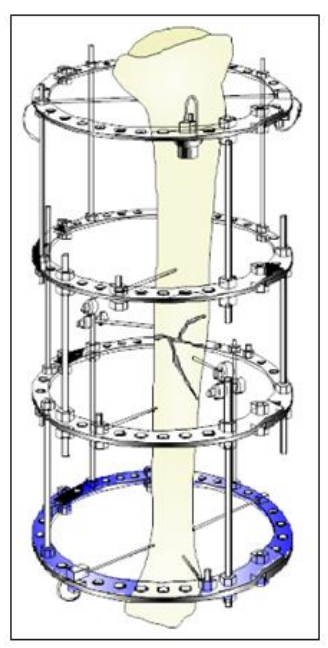

(a)

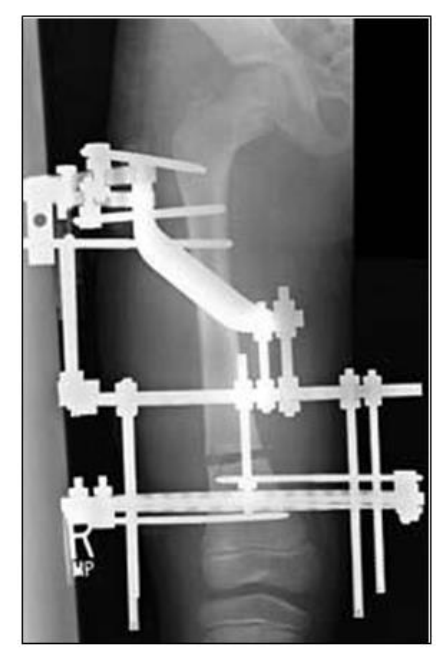

(b)

Fig. 4.1: External fixation: (a) illustration demonstrating external fixator, (b) X-ray picture displaying fracture of femur fixed with external fixator [71].

\subsection{Intramedullary nailing}

Intramedullary (IM) nailing represents the current most popular method for treating femoral diaphysis fractures. The first step in IM fixation is to carefully ream the distal or proximal end of femur to obtain an appropriate size for a nail. Afterwards, a wire is passed across the fracture site to guide the nail in the femur. Then, using lateral screws, the nail is secured in the appropriate position as shown in Fig. 4.2 [77, 78]. 


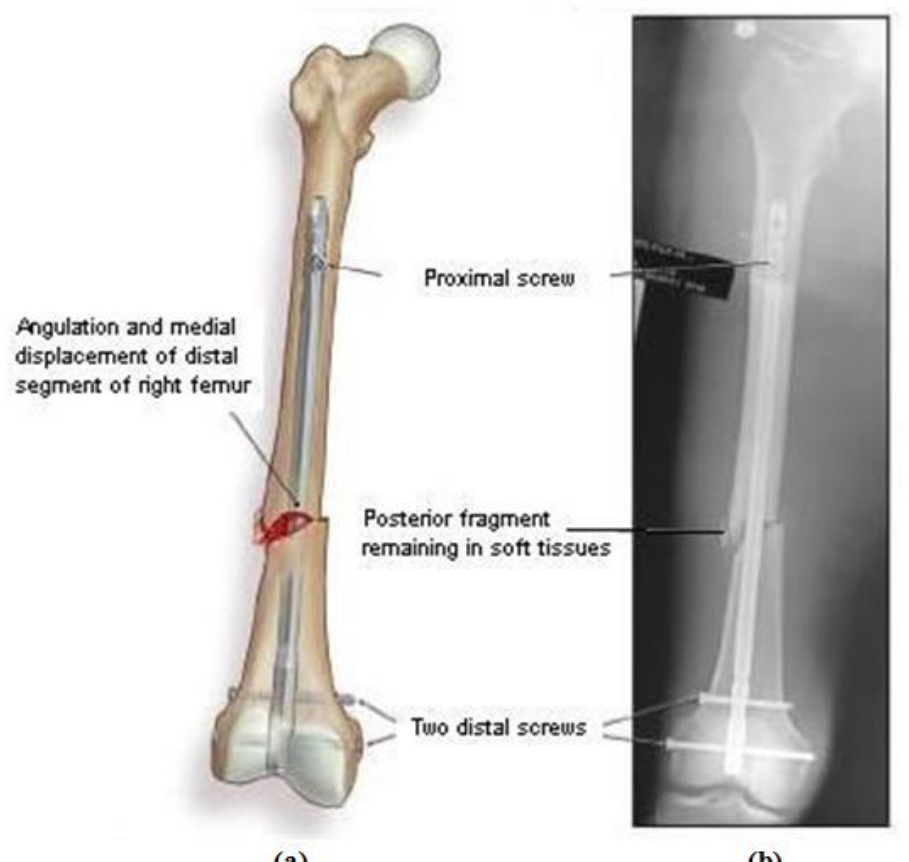

(a)

(b)

Fig. 4.2: Intramedullary nailing: (a) illustration demonstrating completed implant, (b) X-ray picture of retrograde nailing [71].

Considering the femur interior is made up of cancellous bone associated with the epiphysis and the medullary cavity, the rod may be positioned without necessarily removing material from the bone core. This technique is regarded as minimally invasive, preventing further soft tissue injury, and offering the chance for appropriate alignment. In addition, early movement after IM nailing is possible as the nail can bear the body weight. The nail is often removed following full recovery of the fracture [77].

Varying insertion techniques may be applied depending on the fracture type and location. The two primary insertion techniques include antegrade nailing and retrograde nailing [71, 76-78]:

In retrograde nailing, the surgery starts with the medial condyle, afterwards proceeding to the proximal end of the femur. The technique is appropriate for patients who have experienced distal femur fractures. Since the distal femur is relatively easy to access, this technique is ideal when 
attending to patients who are expectant, obese, or have spine trauma $[77,78]$.

Conversely, antegrade nailing is achieved by inserting the nail through either the greater trochanter or the piriformis fossa in a distal direction. Antegrade nailing may be applied in numerous types of fractures, if they are found in the area from just below the lesser trochanter to approximately 7 $\mathrm{cm}$ above the knee joint. It is relatively difficult to access antegrade nailing at the starting point, compared to retrograde nailing, and this is the case particularly for obese or muscular patients. Nevertheless, it has been noted that both healing and infection rates are somewhat similar in the varied insertion starting points [78].

Studies report high healing rates, ranging from 90 to $95 \%$, in intramedullary fixation. However, this operation method may interfere with the growth plate of the femur because of the nature of the operation. Consequently, it is not recommended for patients whose skeletons have not achieved maturity [78].

The intramedullary fixation method presents several major disadvantages [71, 76-78]: First, it may lead to rotational deformity. This fixation method makes use of a nail that passes through the fractured bone, so that the fractured ends of the femur come into contact, consequently reuniting in time. Nevertheless, there is no guidance to check the rotational alignment. This implies that an additional operation may be required in the case of misalignment. Second, intramedullary fixation is not recommended where there are numerous segments and comminutions on the fracture site since it is too difficult to align all the fracture segments. Third, IM nailing may result in a reduction in the overall length of the femur. This side effect could be surgically managed through procedures that either shorten the contralateral femur or lengthen the short femur. Such additional procedures, however, are costly and increase risks as well as time needed for recovery. Fourth, the process of 
reaming of the epiphysis, piriformis fossa or the greater trochanter to achieve the required canal diameter increases fracture risks and may result in damage to the femoral neck. A fracture to the femoral neck is generally regarded as the most serious complication that can arise in the course of intramedullary nailing. Fifth, owing to the nature of the procedure, intramedullary nailing results in the damage of the nutrient artery, following implantation. While revascularization may be recovered via the small vessels in around six weeks, the regeneration of the nutrient artery may require a period of up to six months. Moreover, it is possible to further delay the artery regeneration or permanently damage it where reaming is performed in the nailing [71, 76-78].

\subsection{Plating}

Plating, which is sometimes referred to as bone plate fixation, represents another popular technique applied when repairing a fractured femur. In plating, it is necessary to open up the injured area so that a bone plate can be placed over the fractured bone. The bone plate would be secured by screws or cables, or a combination of the two. Locking bone plates used in the procedure come with threaded screw holes, which facilitate tighter fastening of the bone plates and screws, when compared to non-locking plates [81].

There are typically four types of bone plates, including neutralization plate, buttress plate, compression plate, and condylar plate [76]:

The neutralization plate, shown in Fig. 4.3, is sometimes referred to as the protection plate. Using bone screws, this plate type is installed as a bridge connecting the healthy segments of bone that exist below and above the site of fracture. Such a plate type does not pre-load the fracture site, which is often found in the mid-shaft. In addition, this plate is not supposed to be subjected to 
torsional, shearing, or bending forces. It is generally applied in cases where there are short oblique fractures, fractures with mild fragmenting, or butterfly fractures [76].

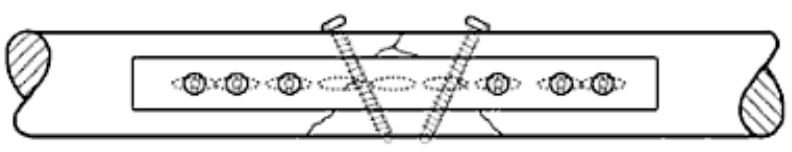

Fig. 4.3: Neutralization plate secured with regular bone screws along with two lag screws [81].

Buttress plates (Fig. 4.4) are often used in managing metaphyseal and epiphyseal fractures, which are usually associated with weakened bone cortex. Buttress plates are designed to have larger surface areas to ensure the load is distributed more widely. It is firmly fastened to the larger bone fragment with bone screws, while being snugly fit to the underlying bone cortex. To apply compression forces across the fragments, cancellous lag screws are used. The force applied to the bone by a buttress plate is in a perpendicular direction in relation to the surface of the plate [76].

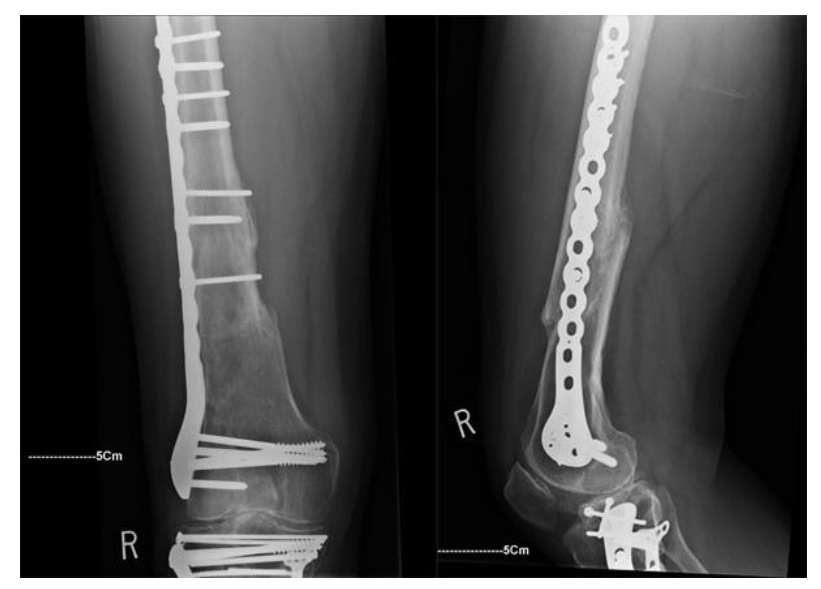

Fig. 4.4: Buttress plate with wide surface area near to the distal epiphysis [81].

Compression plates are applied in order to introduce a compressive pre-load into the fracture site. This is attained by initially fastening the plate on one side of the bone that is fractured. Afterwards, the plate is subjected to tension load through the pulling of the plate free end away from the fixed 
end as shown in Fig. 4.5. The bone plate is then secured to the other side of the bone fracture, while relieving the external pulling force. As soon as the pulling force is removed, the plate attempts to contract to be in the initial dimensions (unloaded), while squeezing the bone halves together. The compressive pre-load is helpful in preventing inter-fragmentary fractures under shear or torsional loading condition and prevents the opening of the fracture surfaces. It further protects the blood supply system, and could reduce the required healing time [72].

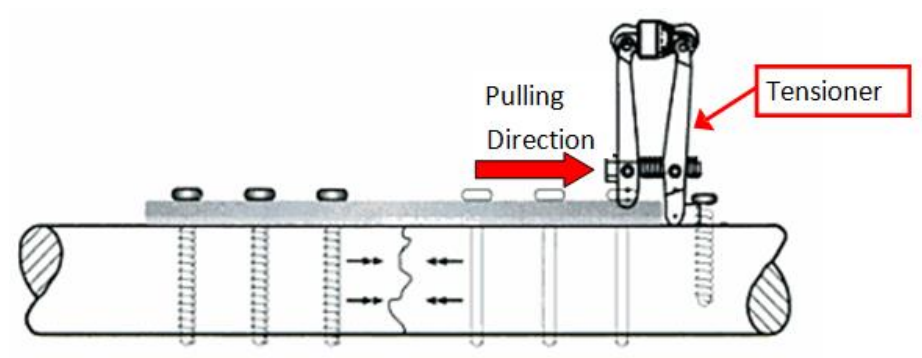

Fig. 4.5: Compression plate secured with bone screws and pulled by tensioner [81].

Intra-articular distal femoral fracture with metaphyseal comminutions are treated by condylar plates which illustrated in Fig. 4.6. The design of the condylar plate is similar to that of the buttress plate, although the condylar plate is applied when a restoration of the knee joint is needed. So as to achieve a knee joint restoration, the plate fastens the metaphysis to the femoral shaft, while carrying out the functions of both a neutralization plate and buttress plate. Similar to compression plate, this type of plate can be attached to the bone with a tensioner to inhibit fracture site opening [76]. 


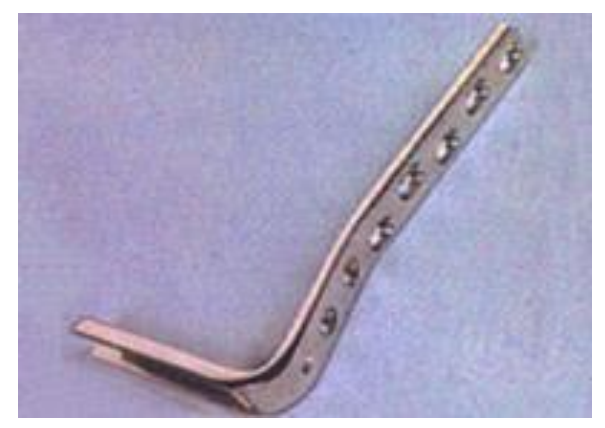

Fig. 4.6: a sample of condylar plate [72].

Most of the bone plates used commercially are manufactured from metals, including titanium alloys, cobalt alloys as well as stainless steel [72]. Since the characteristics of these metals are widely recognized, and their methods of manufacturing have been greatly developed to come up with greatly precise geometries, these metals are popular. However, the high stiffness associated with metallic bone plates, implies that the bone plates carry a large fraction of the load (stress shielding), therefore the bone becomes weak and less dense even following full union at the site of fracture and then will be prone to re-fracturing upon removal of the bone plate $[72,76]$.

There are other major disadvantages associated with bone plate fixation. First, the risks of infection are higher in bone plate fixation than in intramedullary nailing. This is because of the large amount of hardware demanded in bone plate fixation, in the form of screws and cables. Consequently, highly meticulous sanitization processes have to be integrated in the preparation stages before operation. In addition, the appropriate antibiotics have to be prescribed to manage infections and any serious illnesses that may arise thereafter. Furthermore, plating is associated with high risks of non-union, for example, due to hardware malfunction. In plating, the cables and screws employed are more likely to fail, compared to IM nailing. In cases where the hardware does not adequately maintain alignment of the fracture surface, surgical correction is immediately required [77]. 


\subsection{Total hip arthroplasty (THA)}

In total hip arthroplasty (THA), a prosthesis implanted in the body replaces the damaged hip joint. THA consists of four parts, as shown in Fig. 4.7. They include [43]:

- A femoral stem which attaches to the shaft of the bone. It can be either cemented or press fit into the femur.

- A metallic femoral ball (head) which is placed on the upper part of the femoral stem to replace the head of the femur.

- A metallic socket (acetabular component) which replaces the damaged cartilage surface of the acetabulum.

- A plastic or ceramic cup (liner), which is positioned between the socket and femoral ball to allow for a smooth contact surface.
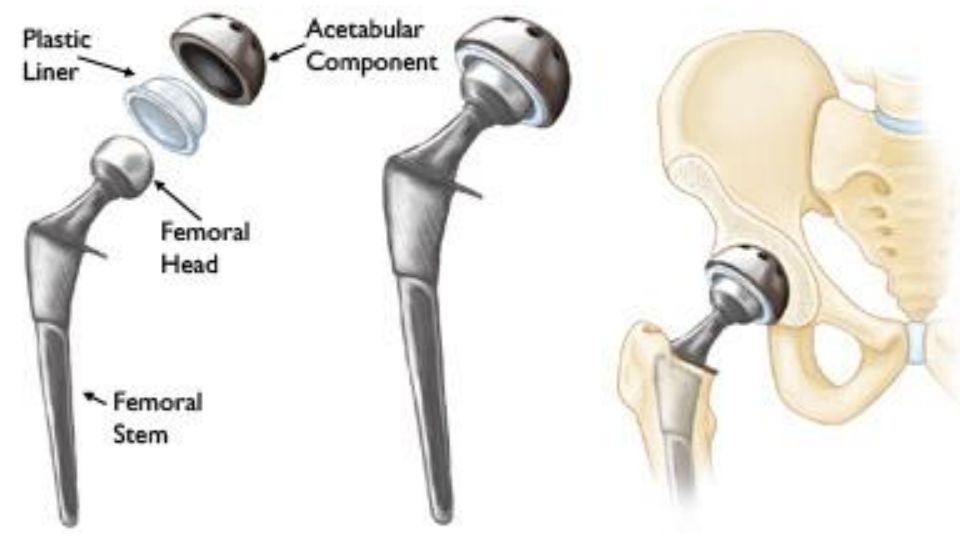

Fig. 4.7: The components of hip joint prosthesis [43].

There are two main reasons for carrying out a total hip arthroplasty: To relieve pain, and to improve function in the joint. Following anesthetization of the patient, a surgeon makes 8-12 inches incision 
down the side of the upper thigh of the patient. Afterwards, the surgeon may opt to install the joint from the back, the front, or the side. The surgeon then separates muscles and

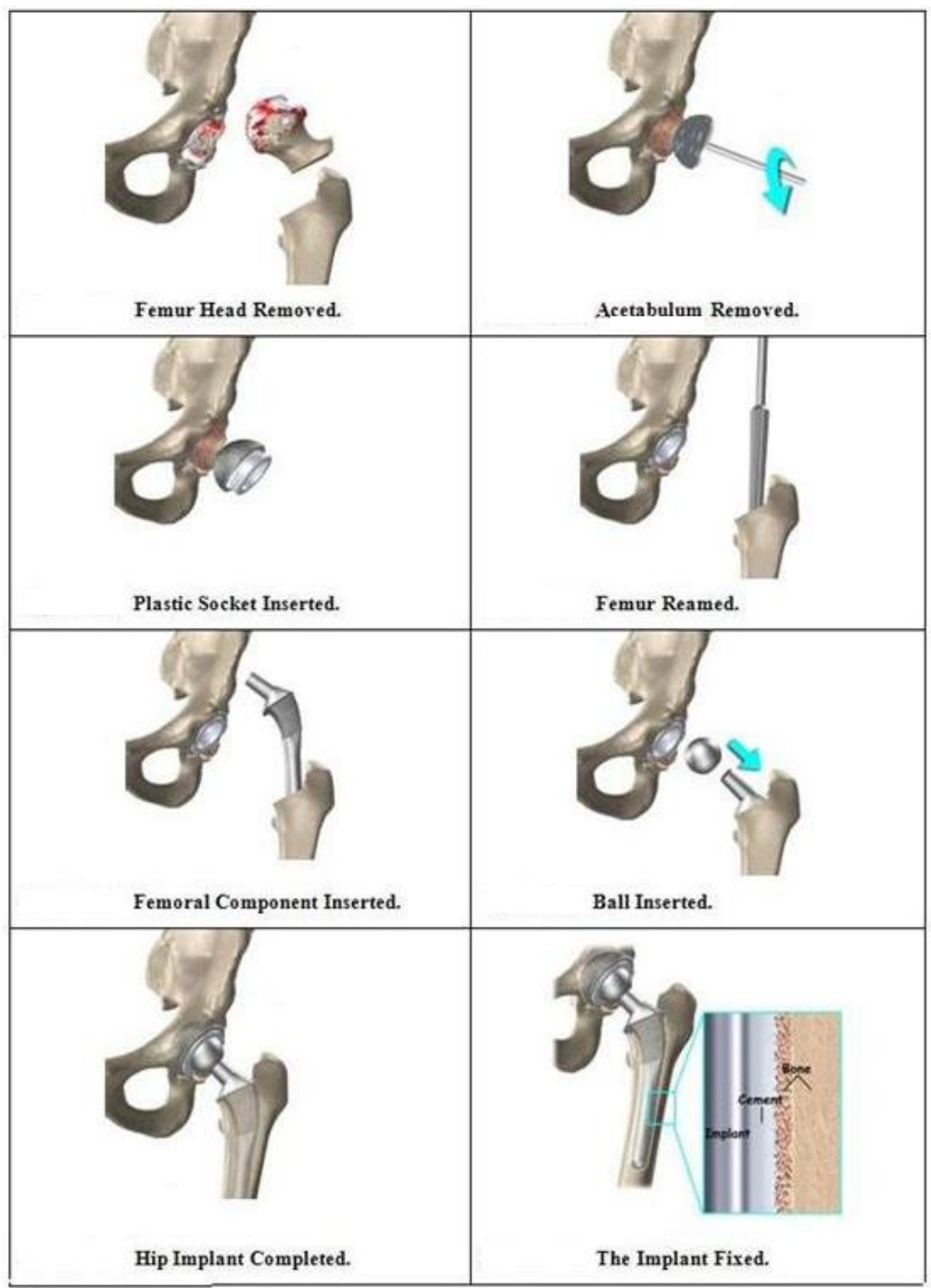

Fig. 4.8: Total hip arthroplasty procedure [43].

ligaments under the skin. When in the joint area, the surgeon separates the femoral head from the acetabulum, and detaches the head from the femoral shaft using a saw (Fig. 4.8). The surgeon then removes cartilage in the acetabulum by employing a power drill and a specialized reamer, he shapes the acetabulum appropriately to facilitate fitting of the acetabular part of the prosthesis into 
the joint. The surgeon then installs the acetabular component after selecting an appropriate size for the patient and then drills a hollow section within the shaft that would accept the stem. The stem could be cemented in place or secured in the position by tightness of fit. The femoral ball is then attached to the stem and placed into the acetabular component. After installing all the components, it is critical to do an X-ray scan to ensure that the prosthesis has been appropriately positioned. After 10 to 14 days following surgery, the staples may be removed [43]. 


\section{CHAPTER 5 : MATHEMATICAL FORMULATION OF THE MECHANO- BIOCHEMICAL MODEL (THERMODYNAMIC-BASED MODEL)}

\subsection{Thermodynamic-based model (Mechano-biochemical model)}

In the thermodynamic-based model (developed by [6] and modified by [7, 8]), bone resorption and formation phases were considered the steps of bone remodeling mechanisms and bone was hypothesized as a thermodynamic system exchanging matter, energy and entropy with its surroundings. It was assumed that the bone remodeling process is performed by biochemical reactions which are introduced in the following section.

\subsubsection{Biochemical reactions involved in bone remodeling}

In the mechano-biochemical model, it is considered that the mechanism of bone remodeling are performed by five biochemical reactions i.e. formation of multinucleated osteoclasts, old bone decomposition, production of osteoblast activator, osteoid production, and calcification $[6,8,13$, 82, 83] as detailed below:

Osteoclasts are the only cells able to resorb bone tissues. These cells initially exist as mononuclear cells, MCELL. In order to activate, mononuclear cells have to be coupled into multinucleated osteoclasts, $M N O C$, whose formation can be characterized by the following reaction.

$$
(\alpha=1) \quad N_{1}+M C E L L \stackrel{K_{+1}}{\underset{K_{-1}}{\leftrightarrows}} M N O C+N_{4}
$$

$N_{l}$ is the mixture of substances initiating the reaction (e.g. nitric oxide) with the mononuclear cells. $N_{4}$ is the remaining product from the first reaction $(\alpha=1)$. 
In the next step $(\alpha=2)$, which is so-called bone decomposition, multinucleated osteoclasts act on the bone to break it down:

$$
(\alpha=2) \quad M N O C+O l d \_B \underset{K_{-2}}{\stackrel{K_{+2}}{\rightleftarrows}} N_{6}+N_{7}
$$

where $O l d \_B$ is the abbreviation for the old bone. $N_{6}$ and $N_{7}$ are the products resulting from the degradation of the old bone. Then, as described in the following reaction $(\alpha=3), N_{7}$ is used in the production of osteoblasts activator (Activ_OB).

$$
(\alpha=3) \quad N_{7}+O_{d} B B \underset{K_{-3}}{\stackrel{K_{+3}}{\rightleftarrows}} \text { Activ_OB }+N_{9}
$$

In the next reaction $(\alpha=4)$, the activators act on the osteoblasts, $O B$, which causes them to secrete collagen and produce Osteoid which is an unmineralized bone:

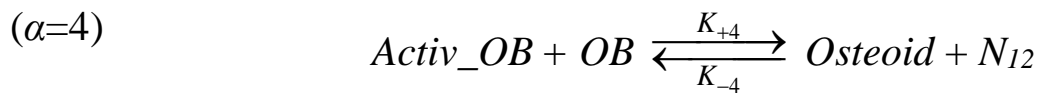

where $N_{9}$ and $N_{12}$ are the remaining product of the $3^{\text {rd }}(\alpha=3)$ and $4^{\text {th }}(\alpha=4)$ reaction, respectively.

The last and longest reaction $(\alpha=5)$ in the bone remodeling process is the mineralization of the Osteoid. $N_{13}$, the substrate which initiates bone calcification, is embedded into the bone matrix to form the new bone $\left(N e w \_B\right)$ according to:

$$
(\alpha=5) \quad N_{13}+\text { Osteoid } \underset{K_{-5}}{\stackrel{K_{+5}}{\rightleftarrows}} N e w_{-} B+N_{15}
$$

where $N_{15}$ is the remaining substrate in the bone formation reaction. 
Equations (5-1) to (5-5) contain 15 substances $\left(N_{1}\right.$, MCELL, MNOC,$N_{4}$, Old $_{-} B, \ldots$ and $\left.N_{15}\right)$ whose concentrations will be denoted by $\left[N_{1}\right],[$ MCELL $],[$ MNOC $],\left[N_{4}\right],\left[\right.$ Old $\left._{-} B\right], \ldots$ and $\left[N_{15}\right]$, respectively.

\subsubsection{Mechano-biochemical coupling}

In the mechano-biochemical model, it is assumed that the coupling between mechanical and biochemical fluxes or forces drives the bone remodeling mechanisms. In order to include the influence of mechanical loading on biochemical reactions, the standard law of mass action $\left(r_{\alpha}=\right.$ $l_{\alpha \alpha} A_{\alpha}$; when the effect of loading is not incorporated) was replaced by a modified version represented by Eq. (5-6) which takes into account the applied mechanical load and biochemical affinity of the reactions $[6,8,13,82,83]$.

$$
r_{\alpha}=l_{\alpha \alpha} A_{\alpha}+l_{\alpha \nu} d_{(1)}=k_{+\alpha} \prod_{i=1}^{n}\left[N_{i}\right]^{v_{\alpha i}}-k_{-\alpha} \prod_{i=1}^{n}\left[N_{i}\right]^{v_{\alpha i}^{\prime}}+l_{\alpha \nu} d_{(1)}
$$

where the rate and affinity of the $\alpha^{\text {th }}$ biochemical reaction ( $\alpha$ is the reaction number) are denoted by $r_{\alpha}$ and $A_{\alpha}$, respectively. $d_{(1)}$ denotes the first invariant of the strain rate tensor, representing the rate of volume change. Phenomenological and reaction rate coefficients are shown by $l$ and $k_{ \pm \alpha}$, respectively. $v_{\alpha i}$ and $v_{\alpha i}^{\prime}$ are the stoichiometric coefficients of the mixture of $N_{i}$ entering and leaving the $\alpha^{\text {th }}$ reaction, respectively. The concentration of $N_{i}$ is denoted by $\left[N_{i}\right]$.

The reaction rate calculated by Eq. (5-6), was then incorporated into Eq. (5-7) [13] which describes the time changes of molar concentration $\left(\left[\dot{N}_{i}\right]\right)$ in terms of stoichiometric coefficients $\left(v_{\alpha i}\right.$ and $v_{\alpha i}^{\prime}$ ) , reaction rate $\left(r_{\alpha}\right)$ and substrate flux $\left(j_{i}\right)$ 


$$
\left[\dot{N}_{i}\right]=\sum_{\alpha=1}^{5}\left(v_{\alpha i}^{\prime}-v_{\alpha i}\right) \cdot r_{\alpha}+j_{i}
$$

The aforementioned substitution provided a system of differential equations describing the mechanism of bone remodeling. In other words, using the modified version of the law of mass action, the time evolution of the concentration of biochemical substances involved in the bone remodeling mechanisms was described by a set of differential equations. Assuming the fluxes are constant in time and the forward reactions are dominant in all considered biochemical reactions, the system of differential equations (Eq. (5-8) to (5-12)), and subsequently their stationary solutions (Eq. (5-13) to $(5-17)[6,8,13])$ for five independent variables were obtained.

$$
\begin{aligned}
& \overline{[M C E L L]}=-\delta_{1}\left(\beta_{1}+\overline{[M C E L L]}\right) \overline{[M C E L L]}+J_{3}+J_{14}-D_{1} \\
& \overline{\left[\text { Old }_{-} B\right]}=-\left(\beta_{3}-\overline{[M C E L L]}+\overline{\left[O l d_{-} B\right]}+\overline{\left[\text { Activ } \_B\right]}+\overline{[\text { Osteoid }]}+\overline{\left[\text { New }_{-} B\right]}\right) . \\
& \left.\left.\overline{\left[O l d_{-} B\right.}\right]-\delta_{3}\left(\beta_{7}-\overline{\left[O l d_{-} B\right.}\right]-2\left(\overline{\left[\text { Activ } \_B\right]}+\overline{[\text { Osteoid }]}+\overline{\left[N e w_{-} B\right]}\right)\right) . \overline{\left[O l d_{-} B\right]}+ \\
& 2 J_{14}-D_{2}-D_{3} \\
& \overline{\left[\text { Activ }_{-} O B\right]}=\delta_{3}\left(\beta_{7}-\overline{\left[O l d_{-} B\right]}-2\left(\overline{\left[\text { Activ } \_B\right]}+\overline{[\text { Osteoid }]}+\overline{\left[\text { New }_{-} B\right]}\right)\right) . \\
& \overline{\left[O l d_{-} B\right]}-\delta_{4}\left(\beta_{10}-\overline{[\text { Osteoid }]}-\overline{\left[N e w_{-} B\right]}\right) \overline{[\text { Activ_OB }]}+D_{3}-D_{4} \\
& \overline{[\text { Osteoid }]}=\delta_{4}\left(\beta_{10}-\overline{[\text { Osteoid }]}-\overline{\left[\text { New_B }_{-}\right]}\right) \overline{[\text { Activ_OB }]} \\
& -\delta_{5}\left(\beta_{13}-\overline{[\text { New_B }]}\right) \overline{[\text { Osteoid }]}+D_{4}-D_{5} \\
& \overline{\left[N e w_{-} B\right]}=\delta_{5}\left(\beta_{13}-\overline{\left[N e w_{-} B\right]}\right) \overline{[\text { Osteoid }]}-J_{14}+D_{5}
\end{aligned}
$$




$$
\begin{aligned}
& \overline{[M C E L L]}=\frac{1}{2}\left(-\beta_{1}+\sqrt{\beta_{1}^{2}+4 \frac{-D_{1}+J_{3}+J_{14}}{\delta_{1}}}\right) \\
& \left.\overline{\left[O l d_{-} B\right]}=\frac{1}{2}\left(\sqrt{\left(\beta_{7}+2 \beta_{3}-2 \overline{[\overline{M C E L L}]}\right)^{2}+4\left(\frac{J_{14}-D_{3}}{\delta_{3}}+2 J_{14}-2 D_{2}\right)}\right)\right) \\
& \overline{\left[\text { Activ_OB] }_{-}\right.}=\frac{1}{2}\left(\sqrt{\left(\sqrt{\left(\beta_{10}+\frac{1}{2}\left(\overline{\left[O l d_{-} B\right]}-\beta_{7}+\frac{J_{14}-D_{3}}{\delta_{3} \overline{\left[O l d_{-} B\right]}}\right)\right)^{2}+4 \frac{J_{14}-D_{4}}{\delta_{4}}}\right)}\right)
\end{aligned}
$$

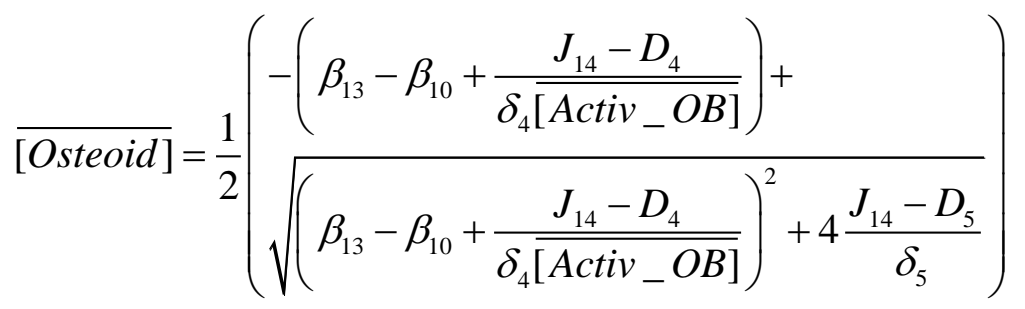

$$
\begin{aligned}
& \overline{\left[N e w_{-} B\right]}=-\overline{[\text { Osteoid }]}+\beta_{10}-\frac{J_{14}-D_{4}}{\delta_{4} \overline{[\text { Activ_OB }]}}
\end{aligned}
$$

where $\overline{\left[N_{i}\right]}$ and $\overline{\left[N_{i}\right]}$ are the normalized time changes of concentration and normalized concentration of $N_{i}$, respectively. $\beta_{i}$ is the sum of the initial molar concentration of relevant substances. $\delta_{\alpha}$ denotes the ratio of the rate of $\alpha^{\text {th }}$ reaction to the second reaction. $D_{\alpha}$ represents the effect of mechanical loading (an approximation of $d_{(1)}$ ) on the $\alpha^{\text {th }}$ biochemical reaction which is expressed as follows $[6,8,13,82,83]$

$$
D_{\alpha}=\frac{1}{\Delta t} \frac{\left|\varepsilon_{(1)}\right|}{S(\text { ref })} D_{\alpha}(\text { ref })
$$


where $\Delta t$ is the time of loading, $\varepsilon_{(1)}$ is the trace of strain tensor, $S(r e f)$ and $D_{\alpha}(r e f)$ are constants describing the reference strain rate value and the influence of the reference strain rate on the $\alpha^{\text {th }}$ reaction, respectively. The values of model parameters which characterize the biochemical reactions have been provided in an earlier work [8].

\subsection{Density and elastic modulus}

The bone density $(\rho)$ is related to the initial bone density $\left(\rho_{0}\right)$, normalized concentration of the new bone $\left(\overline{\left[\mathrm{New} w_{-} \mathrm{B}\right]}\right)$ and old bone $\left(\overline{\left[\mathrm{Old} d_{-} \mathrm{B}\right]}\right)$ according to the following law of mass and mixture [13]:

$$
\rho=\rho_{0} \cdot\left(\overline{\left[O l d_{-} B\right]}+\overline{\left[N e w_{-} B\right]}\right)
$$

In the literature, different empirical and experimental relationships between the elastic modulus and density of the bone have been provided [10]. Using a power law relation between the bone density and Young's modulus, the elastic modulus of the bone was calculated by Eq. (5-20).

Helgason et al. experimentally determined that the exponent of density in power law relation is site-specific with a magnitude between 1.4 and 3 corresponding to cancellous and cortical bones, respectively [10]. In the current study, the magnitude of 2 was used to describe the properties of both cortical and cancellous bone.

$$
E=\left[E_{\mathrm{Old}_{-} B} \cdot\left(\frac{\overline{\left[O l d_{-} B\right]}}{\overline{\left[\mathrm{Old} d_{-} B\right]}+\overline{\left[N e w_{-} B\right]}}\right)+E_{\mathrm{New}_{-} B} \cdot\left(\frac{\overline{\left[N e w_{-} B\right]}}{\overline{\left[O l d_{-} B\right]}+\overline{\left[N e w_{-} B\right]}}\right)\right]\left(\frac{\rho}{\rho_{0}}\right)^{2}
$$

where $E_{\text {Old } \_B}$ and $E_{N e{ }_{-} B}$ are the moduli of elasticity of old and new bone, respectively. 


\section{CHAPTER 6 : FINITE ELEMENT MODELING}

\subsection{Development of computer aided design (CAD) models}

\subsubsection{CAD model of intact femur}

A large left $4^{\text {th }}$ generation composite femur (model 3406, sawbones, Vashon, WA, USA) was fully scanned by GE LightSpeed VCT 64 Slice CT scanner $(0.5$ x $0.5 \mathrm{~mm} /$ pixel resolution, and $0.5 \mathrm{~mm}$ slice thickness). The CT scan images were saved in DICOM format and imported into Mimics software (The Materialise, Leuven, Belgium) to generate a three-dimensional (3D) model, and subsequently to create two distinct bodies (i.e. cortical and cancellous). The 3D CAD model was then imported into SolidWorks software (SolidWorks Corp., Dassualt Systèmes, Concord, MA, USA) for virtual implantations (Fig. 6.1) [83].

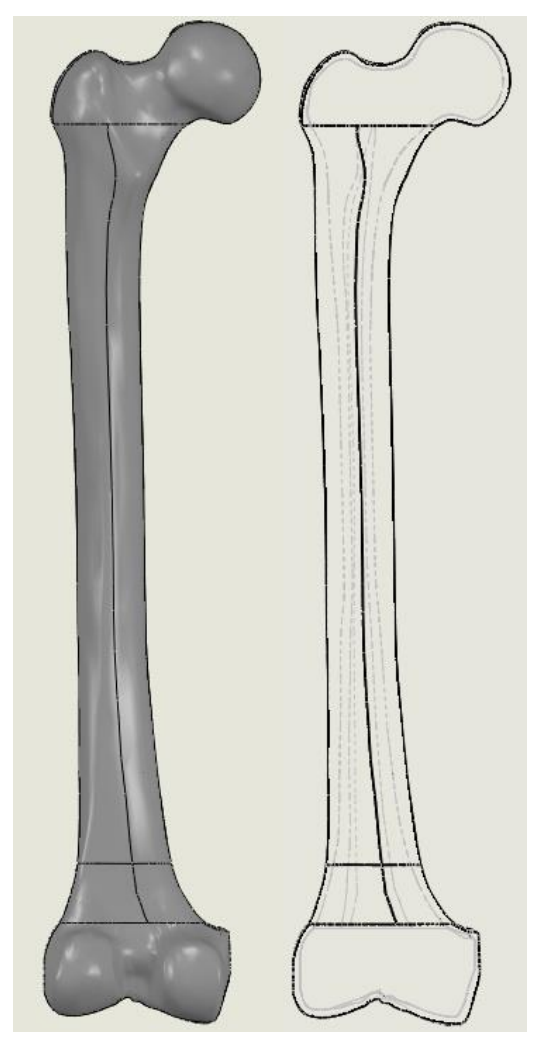

Fig. 6.1: CAD model of the intact femur. 


\subsubsection{CAD model of implants}

To generate the CAD model of the complex geometry of Stryker Exeter hip stem (size 2, offset $37.5 \mathrm{~mm}$ ), unconventional hip implant developed by [9] and Stryker T2 femoral nail (length: 420 mm), NextEngine 3D scanner (NextEngine, Inc, Santa Monica, CA, USA) was employed. This scanner can quickly create highly detailed, full color digital models measuring at a speed of 50,000 points per second with multi-laser precision.

The CAD model of the screws and 8-hole Zimmer fixation plate (length: $246 \mathrm{~mm}$ ) was generated based on the specimen geometry in the experimental trials using a vernier caliper and SolidWorks. The developed CAD models are shown in Fig. 6.2.

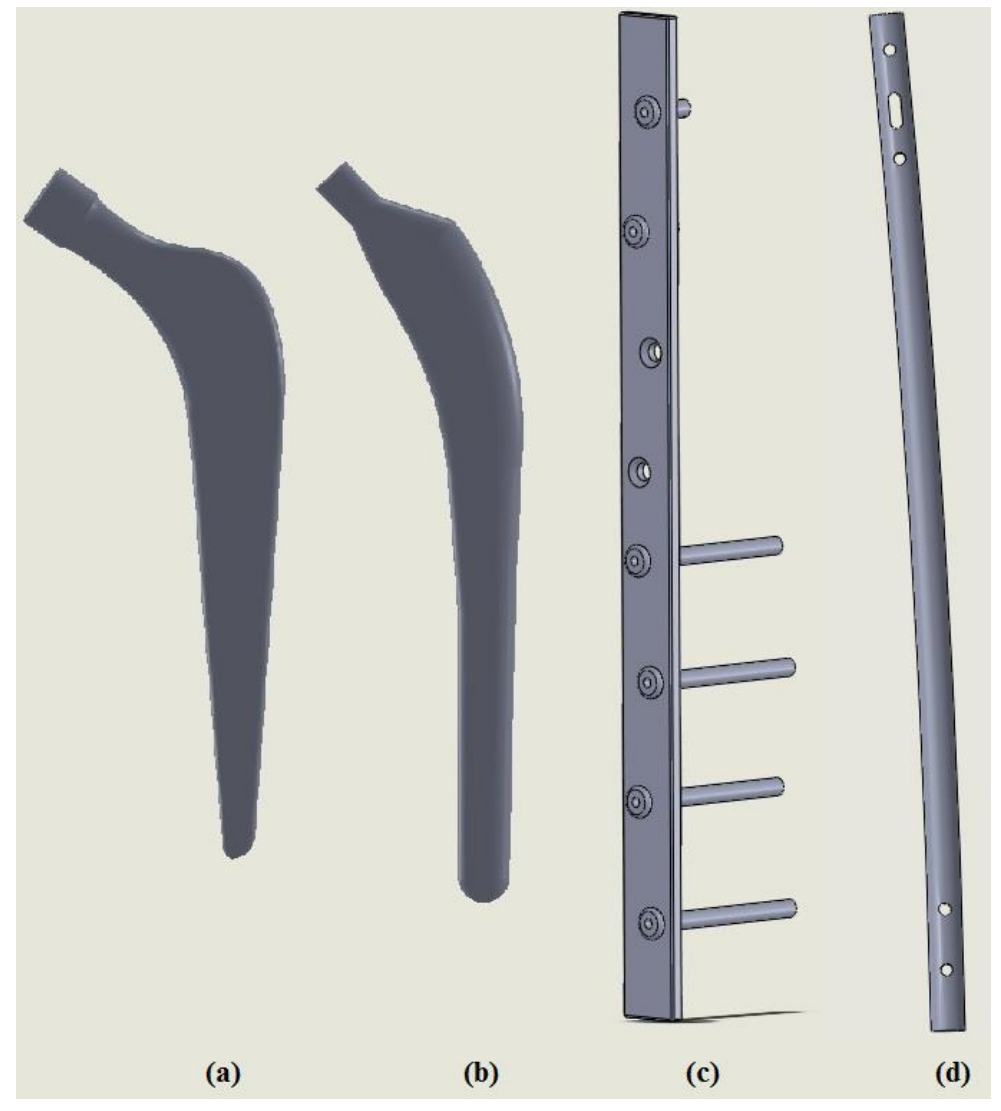

Fig. 6.2: CAD model of: (a) conventional hip implant (Stryker Exeter), (b) unconventional hip implant, (c) bone fracture plate (8-hole Zimmer) with screws, (d) IM nail (Stryker T2 nail). 


\subsubsection{CAD model development of conventional hip implanted femur}

The developed CAD model of intact femur was imported into SolidWorks for virtual implantation with Stryker Exeter hip stem (size 2, offset $37.5 \mathrm{~mm}$, made up of CoCrMo). The femoral head (and neck) was split from the rest of the femur by using the split features at an angle of 55 degrees with respect to the transverse anatomical plane along the greater trochanter. A portion of the proximal cancellous bone was removed to simulate surgically-generated bone loss during total hip replacement. Therefore, we created a CAD model (Fig. 6.3) of femur whose femoral shaft is used for both pre- and post-operative bone remodeling simulations. This enabled us to make a precise comparison between the density distribution of the intact (pre-operative) and post-operative femurs.

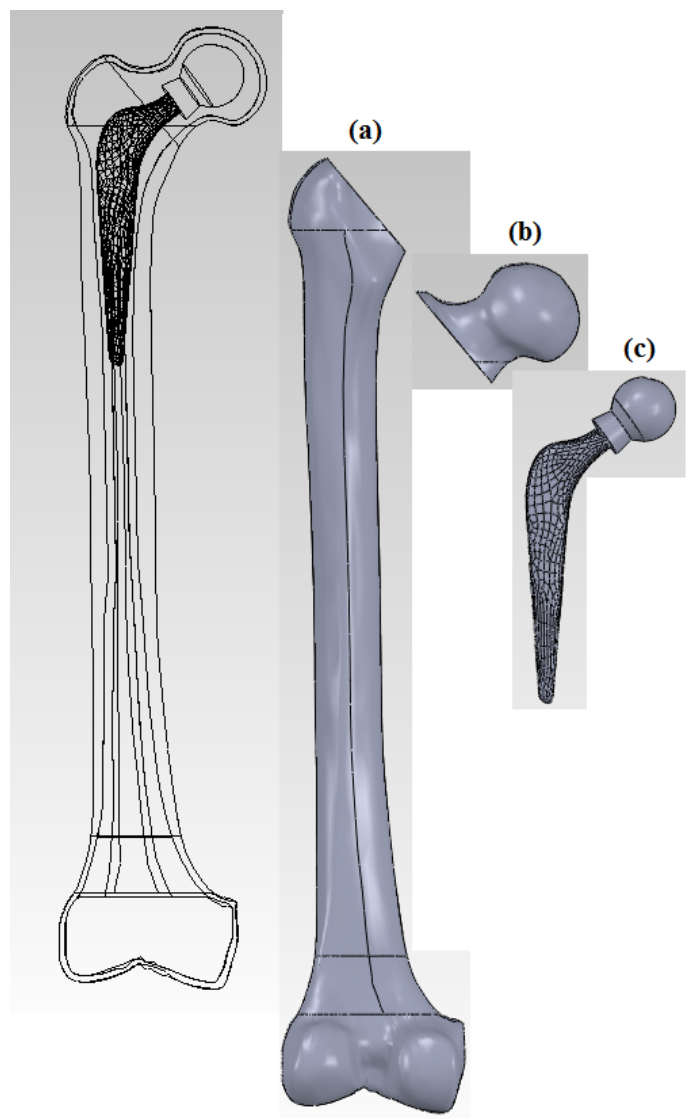

Fig. 6.3: CAD model of: (a) femoral shaft, (b) femoral head and neck, (c) conventional hip implant. 


\subsubsection{CAD model development of unconventional hip implanted femur}

The unconventional composite hip stem developed by [9] is composed of a $3 \mathrm{~mm}$ thick substructure made of CF/PA12 lamina with the stacking sequence of $\left( \pm 45^{\circ}\right)_{6}$ and an internal polymeric core [9]. The geometry of the unconventional metallic hip stems was the same as the composite one; however, the related material properties (CoCrMo or Ti6Al4V) were assigned to the substructure as well as to the internal core in finite element analysis (FEA). In order for virtual implantation, using SolidWorks, the femoral head (and neck) was split from the rest of the femur at an angle of 55 degrees with respect to the transverse anatomical plane and a portion of the proximal cancellous bone was removed (Fig. 6.4).

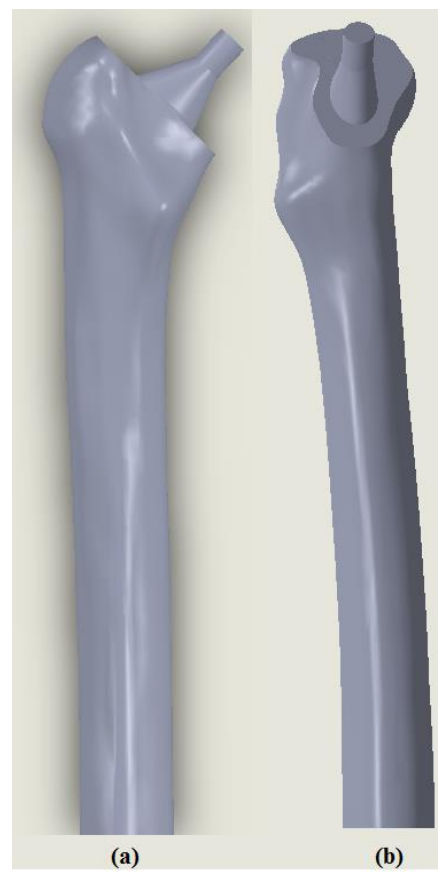

Fig. 6.4: $\mathrm{CAD}$ model of unconventional hip implanted femur: (a) posterior view, (b) medial view.

\subsubsection{CAD model development of hip implanted femur fixed by a bone fracture plate}

Using SolidWorks, a $246 \mathrm{~mm}$-long plate (8-hole Zimmer fixation plate, made up of 316L stainless steel) with three unicortical screws (diameter $4.5 \mathrm{~mm}$; length $14 \mathrm{~mm}$, made up of 316L stainless 
steel) and five bicortical screws (diameter $4.5 \mathrm{~mm}$; length $50 \mathrm{~mm}$, made up of 316L stainless steel) were attached to either lateral or anterior side of the hip implanted femur to mimic fracture fixation constructs for healed femur (post-union) with stable hip implant conditions (Fig. 6.5). It should be mentioned that the conventional hip implant (Stryker Exeter hip stem/size 2, offset $37.5 \mathrm{~mm}$ ) was implanted into the femur by the procedure explained in section 6.1.3.

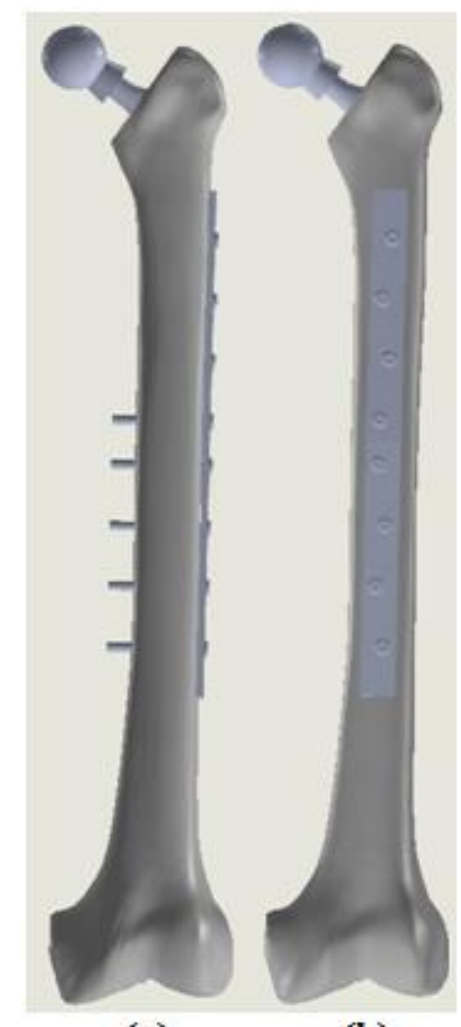

(a)

(b)

Fig. 6.5: CAD model of hip implanted femur with (a) lateral plate, (b) anterior plate.

\subsubsection{CAD model development of femur fixed by an intramedullary (IM) nail}

A $420 \mathrm{~mm}$ Stryker T2 femoral nail made up of Ti6Al4V was modeled as a shell and was antegradely implanted into the CAD model of the femur using SolidWorks (Fig. 6.6). 


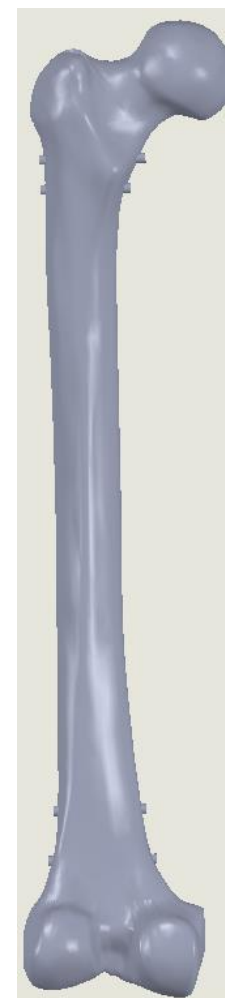

(a)

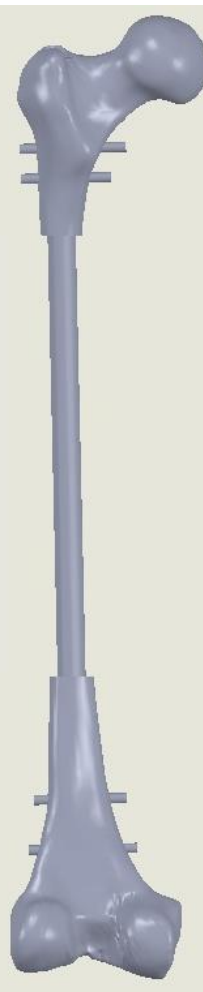

(b)

Fig. 6.6: CAD model of: (a) the femur with the intramedullary nail inside, (b) cortical bone is removed for better visualization.

Table 1: Laminate stacking sequence of composite nail [84].

\begin{tabular}{|c|c|c|c|c|}
\hline Ṡ & Stacking Sequence & EI \\
$\left(N . m^{2}\right)$ & $\begin{array}{c}\text { EA } \\
(M N)\end{array}$ & $\begin{array}{c}\text { G.J } \\
\left(N . m^{2}\right)\end{array}$ \\
\hline C1 & {$\left[-45 / 45_{2} /-45_{2} / 45_{2} /-45_{2} / 45 / 0 / 45 /-45 / 0 / 90_{4}\right]$} & 40.1 & 3.94 & 91.7 \\
\hline C2 & {$\left[-45 / 45_{2} /-45 / 0 /-45 / 45_{2} /-45 / 0 / 45 /-45_{2} / 45 / 90_{4}\right]$} & 51.2 & 3.94 & 85.4 \\
\hline C3 & {$\left[-45 / 45 / 0_{2} / 45 /-45_{2} / 45_{2} /-45_{2} / 45_{2} /-45 / 90_{4}\right]$} & 61.8 & 3.94 & 80.6 \\
\hline C4 & {$\left[0_{2} /-45 / 45_{2} /-45_{2} / 45_{2} /-45_{2} / 45_{2} /-45 / 0 / 90_{2}\right]$} & 70.4 & 4.43 & 78.3 \\
\hline C5 & {$\left[0_{2} /-45 / 45_{2} /-45 / 0 /-45 / 45_{2} /-45_{2} / 45_{2} /-45 / 90_{2}\right]$} & 80.1 & 4.43 & 73.8 \\
\hline C6 & {$\left[0_{2} /-45 / 45 / 0 /-45 / 45_{2} /-45 / 0 /-45 / 45_{2} /-45 / 90_{2}\right]$} & 91.2 & 5.08 & 66.5 \\
\hline C7 & {$\left[0_{4} /-45 / 45_{2} /-45_{2} / 45_{2} /-45_{2} / 45 / 90_{2}\right]$} & 103.0 & 5.08 & 62.0 \\
\hline C8 & Ti-6AL-4V & 132.8 & 6.70 & 98.9 \\
\hline
\end{tabular}


The nail was modeled as shell because the author was interested in comparing bone response to metallic nail with that to composite nails (made up of $\mathrm{CF} /$ Epoxy lamina) with the stacking sequences listed in Table 1. Four $5 \mathrm{~mm}$ locking screws (made up of Ti6Al4V) were modeled as solid bodies and positioned to protrude approximately $2 \mathrm{~mm}$ out of the femur [85]. The intersecting volumes of the bone-nail and bone-screws in the proximal and distal ends were then subtracted from the model.

\subsection{Modeling in ANSYS Workbench}

The prepared CAD models were then imported into ANSYS Workbench 14.5 (ANSYS Inc., Canonsburg, Pennsylvania, USA) for assigning the material properties, applying boundary conditions and meshing.

\subsubsection{Material properties}

The material properties of cortical and cancellous bones, CF/PA12 (lamina), CF/Epoxy (lamina), CoCrMo, Ti6Al4V, 316L stainless steel and composite hip implant polymeric core are provided in Table 2.

It should be mentioned that the stacking sequence and material properties of the composite hip implant and composite nails were assigned to the corresponding structures using ACP (Pre) module of ANSYS Workbench 14.5. 
Table 2: Material properties of CF/PA12, CF/EPOXY, polymeric core, CoCrMo, Ti6Al4V, 316L stainless steel, cortical and cancellous bones $[9,13,16,84]$. L and $\mathrm{T}$ stand for longitudinal (fiber direction) and transverse (normal to fiber direction) directions in the lamina, respectively.

\begin{tabular}{|c|c|c|c|c|c|c|c|c|c|c|c|}
\hline & $\underset{(\mathbf{G P a})}{\mathbf{E}_{\mathbf{L}}}$ & $\underset{(\mathbf{G P a})}{\mathbf{E}_{\mathbf{T}}}$ & $\begin{array}{c}\mathbf{G}_{\mathrm{LL}} \\
(\mathbf{G P a})\end{array}$ & $\begin{array}{c}\mathbf{G}_{\mathrm{TT}} \\
(\mathbf{G P a})\end{array}$ & $\begin{array}{c}\mathbf{G}_{\mathbf{L T}} \\
(\mathbf{G P a})\end{array}$ & $v_{L L}$ & $v_{T T}$ & $v_{L T}$ & $\underset{\text { (GPa) }}{\mathbf{E}}$ & $v$ & Ply Type \\
\hline $\begin{array}{c}\text { CF/PA12 } \\
\text { (lamina) }\end{array}$ & 66.5 & 2.72 & 19.5 & --- & 2.7 & 0.04 & --- & 0.26 & --- & --- & Woven \\
\hline $\begin{array}{c}\text { CF/EPOXY } \\
\text { (lamina) }\end{array}$ & 121 & 8.6 & -- & 3.07 & 4.7 & --- & 0.4 & 0.27 & -- & --- & Prepreg \\
\hline $\begin{array}{l}\text { Polymeric } \\
\text { core }\end{array}$ & --- & --- & --- & --- & --- & --- & --- & --- & 1 & 0.2 & --- \\
\hline CoCrMo & -- & --- & -- & --- & --- & -- & -- & --- & 210 & 0.3 & --- \\
\hline Ti6Al4V & --- & --- & --- & --- & --- & --- & --- & --- & 114 & 0.3 & --- \\
\hline $\begin{array}{c}316 \mathrm{~L} \\
\text { stainless } \\
\text { steel }\end{array}$ & -- & --- & --- & --- & --- & -- & -- & --- & 193 & 0.3 & --- \\
\hline $\begin{array}{c}\text { Cortical } \\
\text { bone }\end{array}$ & -- & -- & --- & --- & --- & --- & --- & --- & 16 & 0.46 & --- \\
\hline $\begin{array}{l}\text { Cancellous } \\
\text { bone }\end{array}$ & --- & --- & --- & --- & --- & --- & --- & --- & 0.155 & 0.3 & --- \\
\hline
\end{tabular}

\subsubsection{Boundary conditions}

In order to simulate the physiological loading, muscle and hip joint reaction forces (Fig. 6.7) [86] regarding $45 \%$ of gait cycle were applied to the model as roughly this instance of the gait produces the maximum loading on the femur [80]. Five major muscles (i.e. m. gluteus minimus, m. gluteus medius, $\mathrm{m}$. gluteus maximus, $\mathrm{m}$. abductor magnus, and $\mathrm{m}$. piriformis) are considered in this muscle system. To avoid stress concentration, muscle and hip joint reaction forces were distributed over several nodes of greater (and lesser) trochanter and femoral head, respectively. To avoid rigid body motion, the degrees of freedom of all nodes at the distal femur were fixed. 
Force: $2941.8 \mathrm{~N}$

B] Force 2: $577.21 \mathrm{~N}$

Force $3: 225.67 \mathrm{~N}$

Fixed Support

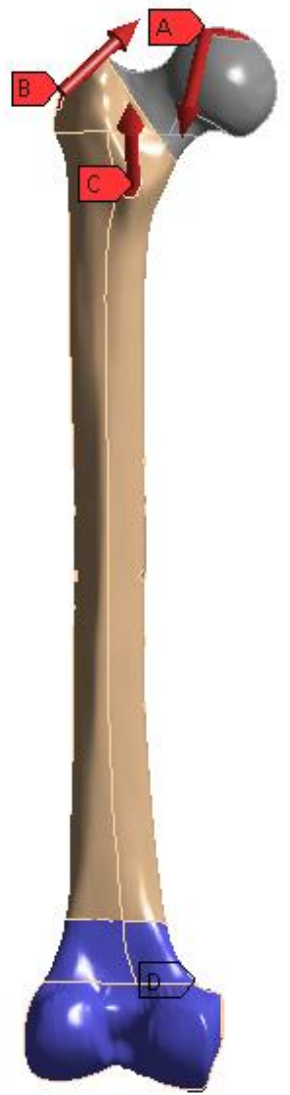

Fig. 6.7: Boundary conditions shown on the typical intact femur.

\subsubsection{Contact types}

Bonded contact type was assigned to the system of hip implant-bone, screws-bone, and screwsplate (or nail). However, the contact between the bone and plate (or nail) was considered as a noseparation which allows for sliding of the touching surfaces. Several studies [71, 87-89] also considered similar assumptions for modeling femoral fracture fixation and total hip arthroplasty. These assumptions are similar to the clinical condition in which bone remodels itself, and simulate perfect interdigitation of bone around the threads of the screws, and full bony in-growth around the hip implant. 


\subsubsection{Element types}

The femoral bone, conventional hip implant, plate, screws and internal core of the unconventional hip implant were meshed by Quadratic Tetrahedrons elements ( SOLID187), whereas the laminates of unconventional hip implant and nail were meshed using Linear Triangle elements $(\sim$ SHELL181). Surface-to-surface contact elements (CONTA174 and TARGE170) were used to mesh all interfaces.

\section{- $\quad$ SOLID187}

SOLID187 (Fig. 6.8) is a higher order 3D tetrahedral solid element with 10 nodes having three degrees of freedom i.e. translation in $\mathrm{x}, \mathrm{y}$ and $\mathrm{z}$ directions at each node. It has quadratic displacement behaviour and is ideal for modeling irregular meshes like those developed by CAD systems, and because of this characteristic, this element type was used to mesh the highly curvaceous geometry of the bones, hip implants, etc. The element has large deflection, large strain, plasticity, hyperelasticity, creep and stress stiffening capabilities. In addition, SOLID187 input data includes the orthotropic or anisotropic material properties which makes this element ideal for complex material behaviour for future studies. Nodal displacement, stresses, strain energy density can be SOLID187 output data [90].

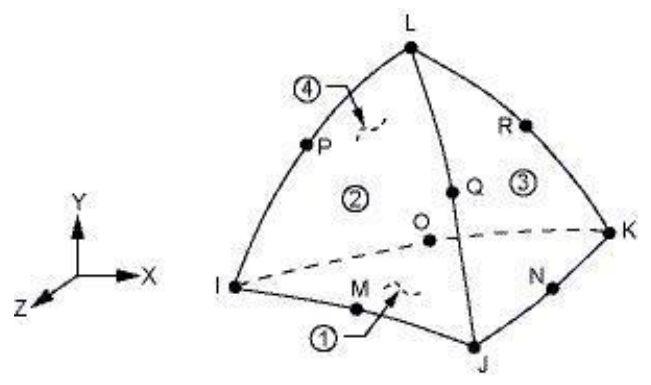

Fig. 6.8: The geometry of SOLID187 element [90]. 


\section{- $\quad$ SHELL181}

SHELL181 (Fig. 6.9) is a 4-node element with six degrees of freedom at each node: rotations about the $\mathrm{x}, \mathrm{y}$ and $\mathrm{z}$-axes, and translations in the $\mathrm{x}, \mathrm{y}$ and $\mathrm{z}$ direction. This type of element is well suited for analyzing thin to moderately-thick shell structures. SHELL181 is applicable for linear, large rotation, and/or large strain nonlinear analysis and can be used for layered applications for modeling sandwich construction or composite shells [90].

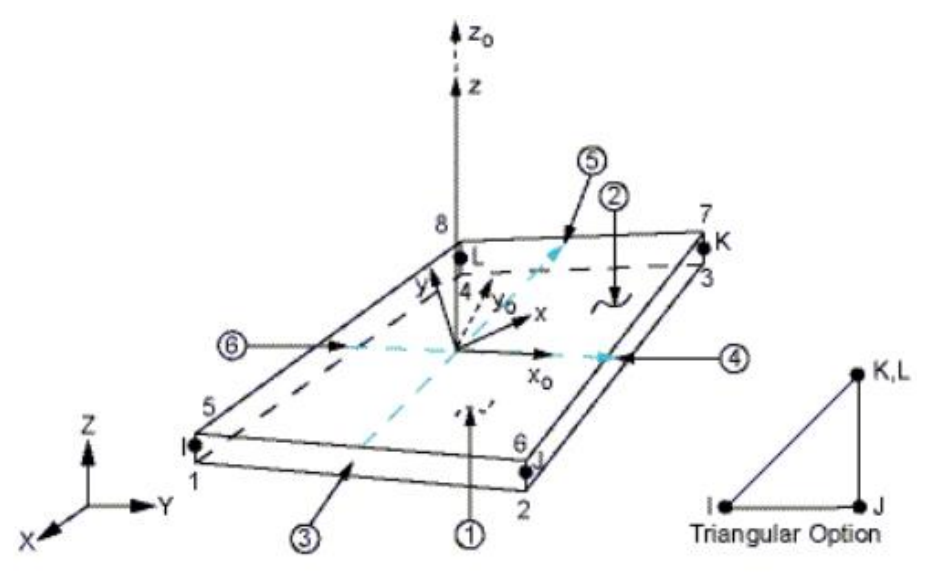

Fig. 6.9: The geometry of SHELL181 element [90].

\section{- CONTA174}

CONTA174 (Fig. 6.10) element type is a 3D 8-node element which is generated automatically for flexible-flexible and rigid-flexible contact analysis. The element is suited for 3D structural and coupled field contacts. CONTA174 is placed on the surfaces of shell or 3D solid elements with mid-side nodes (e.g. SOLID187) and has the same geometric characteristics as the shell or solid element surface to which it is attached. The element can provide contact and sliding between TARGE170 and a deformable surface defined by this element. When the element surface penetrates one of the target segment elements (TARGE170) on a specified target surface, contact 
occurs. The element allows shear stress friction, coulomb friction and also separation of bonded contact for simulating interface delamination [90].

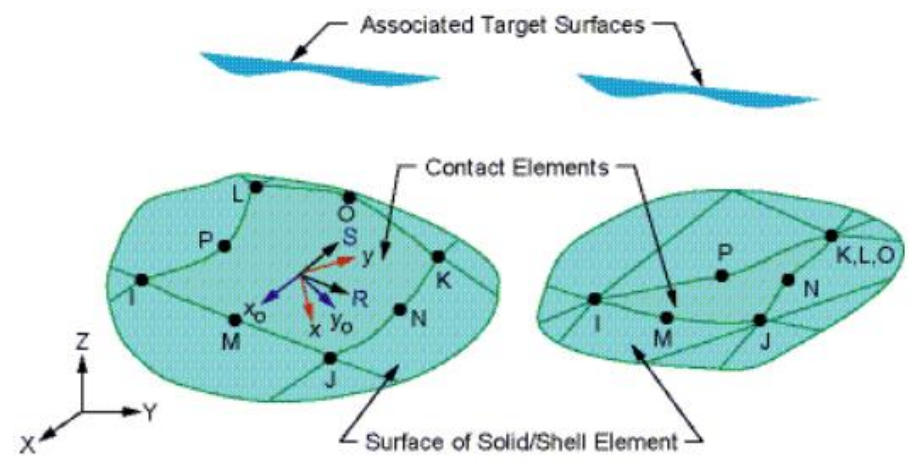

Fig. 6.10: The geometry of CONTA174 element [90].

\section{- TARGE170}

TARGE170 (Fig. 6.11) is a 3D 3-node element which is automatically generated to associate with contact elements (e.g. CONTA174, CONTA173, etc.). Target surface discretized by TARGE170 is in contact with the contact elements (e.g. CONTA174) which overlay the solid or shell elements describing the boundary of a deformable body. It is possible to impose any forces, moments, rotational or translational displacement, magnetic potential, temperature and voltage on the target segment element [90].
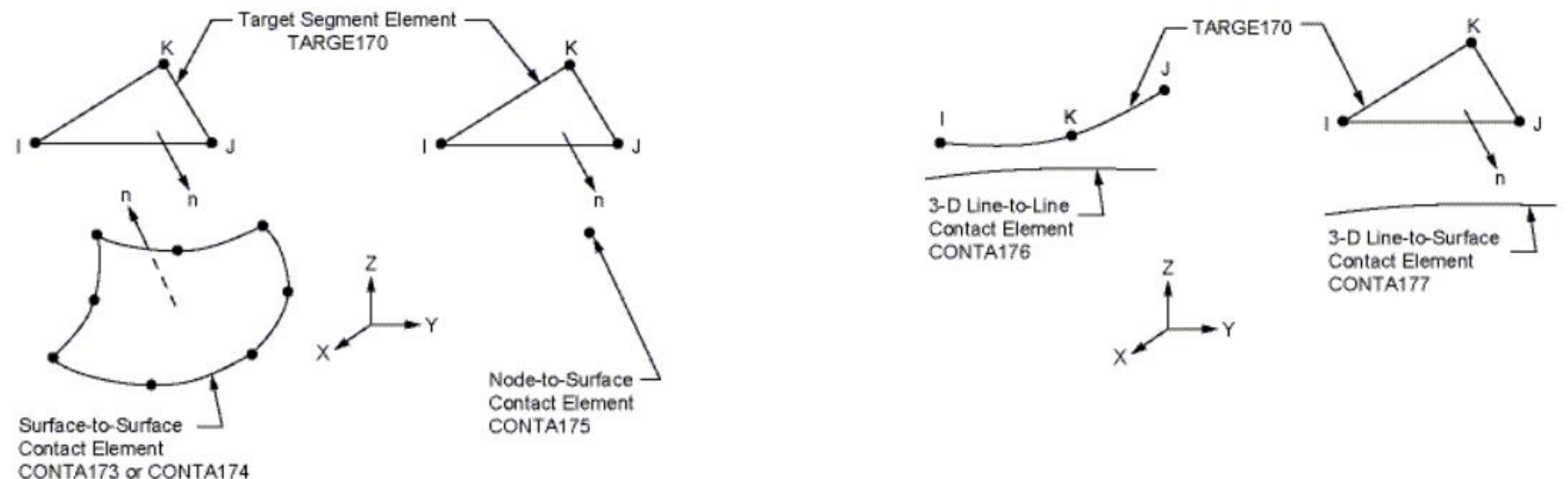

Fig. 6.11: The geometry of TARGE170 element [90]. 


\subsubsection{Mesh sensitivity and meshed constructions}

In the current study, solid bodies (i.e. femoral bone, conventional hip implants, plates, screws and polymeric core of unconventional hip implants) were meshed by 10 -node tetrahedral elements (SOLID187) which can produce a high level of accuracy for the femur biomechanical simulations as verified by [91]. Since the models are mainly constituted of femoral bone, it was fair to expect a sufficient level of accuracy from SOLID187 meshing [72]. Mesh sensitivity was then performed using "Relevance" option of ANSYS 14.5 Workbench, with a value that ranged between -100 indicating a very coarse mesh (high speed mesh) and +100 corresponding to an extremely fine mesh (high accuracy mesh). A value of $90 \%$ was chosen as further increasing the relevance resulted in less than $1 \%$ change in the results i.e. total deformation of a node located on the femoral head. Fig. 6.12 illustrates the typical results of mesh sensitivity analysis for a typical intact femur with boundary conditions stated in section 6.2.2.

The typical meshed constructions i.e. meshed intact, hip implanted (with conventional implant), lateral and anterior plated femurs as well as IM nailed femur is shown in Fig. 6.13.

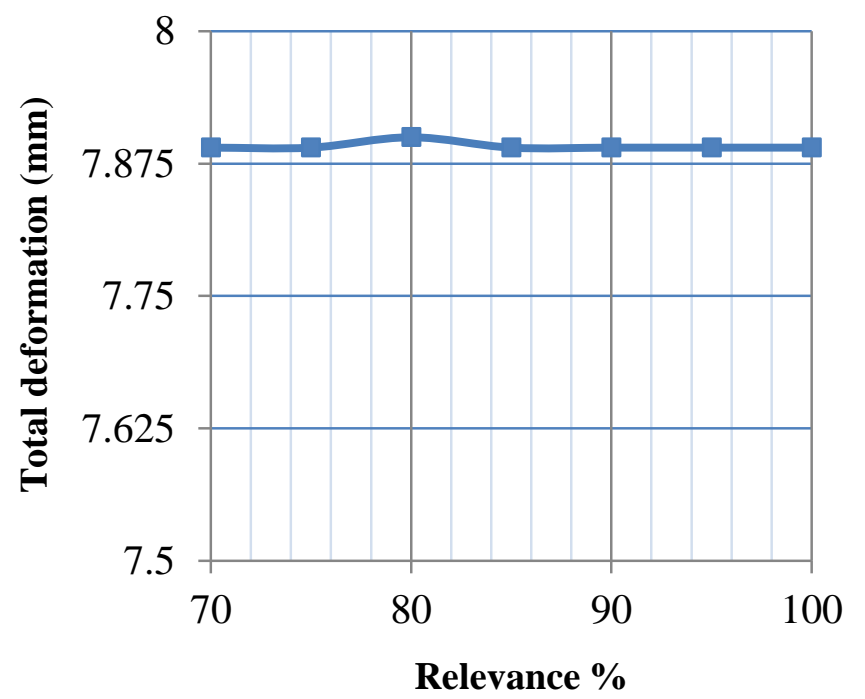

Fig. 6.12: Typical results of mesh sensitivity analysis for the intact femur. 


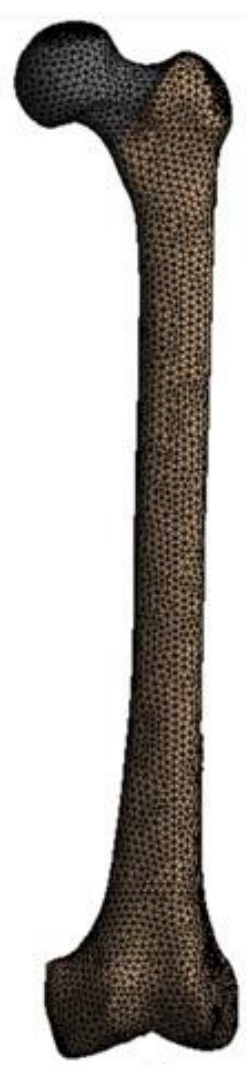

(a)

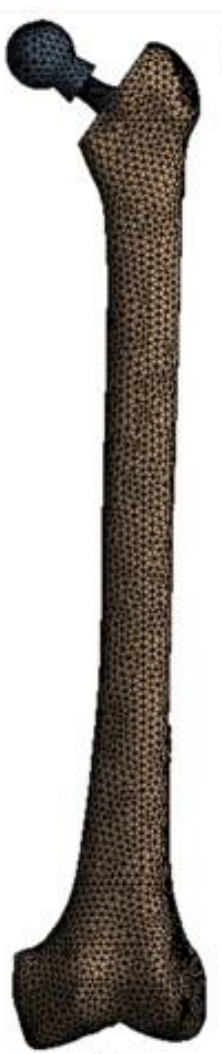

(b)

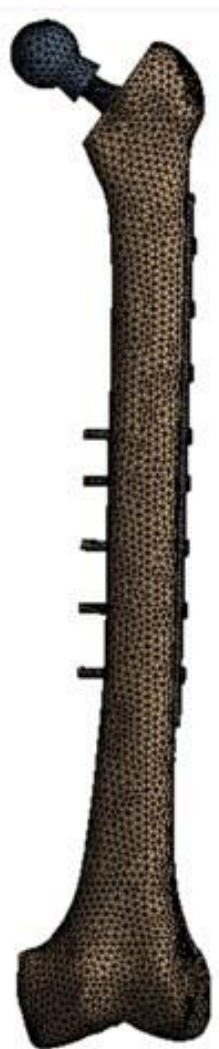

(c)

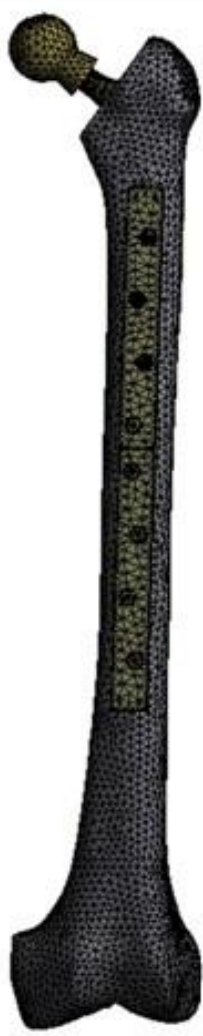

(d)

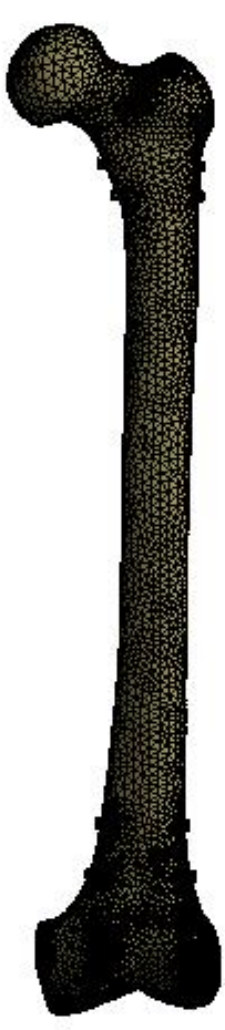

(e)

Fig. 6.13: Typical meshed constructions: (a) intact, (b) hip implanted (with conventional implant), (c) lateral plated, (d) anterior plated and (e) IM nailed femurs.

\subsection{Modeling in ANSYS Parametric Design Language (APDL)}

The algorithm of bone remodeling simulation is illustrated in Fig. 6.14. To simulate bone remodeling using mechano-biochemical model, the meshed models was imported into ANSYS Parametric Design Language (APDL). Forces, constraints and contact types were identical to those in ANSYS Workbench modeling (section 6.2).

In order to obtain bone density distribution throughout the intact femur, in the first iteration of simulations, the bone was modeled as a homogenous structure with the initial density of $0.98 \mathrm{~g} / \mathrm{cm}^{3}$ $\left(\rho_{0}\right)$ which is the average value of cortical bone with $1.64 \mathrm{~g} / \mathrm{cm}^{3}$ and cancellous bone with 0.32 $\mathrm{g} / \mathrm{cm}^{3}$. The mechano-biochemical model is not sensitive to the initial density. In other words, the 
initial density does not have a significant effect on the final bone density distribution similar to other models developed by [5, 92]. To model the intact femur, cancellous and cortical material properties were assigned to their corresponding bodies. The hip implant inside the femur was also considered as cancellous bone.

In the next iterations, based on the concentration of $\mathrm{Old}$ and $\mathrm{New}$ bones remained/produced inside the element, the new properties of each element were calculated. Similar to several studies $[5,14$, $16,93,94]$, each bone element was considered as an isotropic material whose elastic modulus was calculated by Eq. (5-20).

In detail, the mechano-biochemical model which is defined by equations (5-13) to (5-17), was applied to finite element analysis (FEA) through a user-defined macro. FEA yielded the trace of strain tensor, $\varepsilon_{(1)}$, to calculate the values of $D_{\alpha}$ which are required to calculate $\overline{\left[\text { Old } \_B\right]}$ and $\overline{\left[N e w_{-} B\right]}$ for each element. The density and modulus of elasticity of each element were then obtained by Eqs. (5-19) and (5-20), respectively. These new material properties were used for the next iteration, and the process was repeated until no significant change in the density of the elements was observed. It was assumed that the convergence of simulations was obtained when ConvCri $<0.0001$ (Eq. (6-1)).

$$
\operatorname{Conv} C r i=\frac{1}{n} \sum_{i=1}^{n}\left|\rho_{i}^{(t)}-\rho_{i}^{(t-1)}\right|
$$

where $n$ and $t$ are the number of elements and iteration number, respectively. 


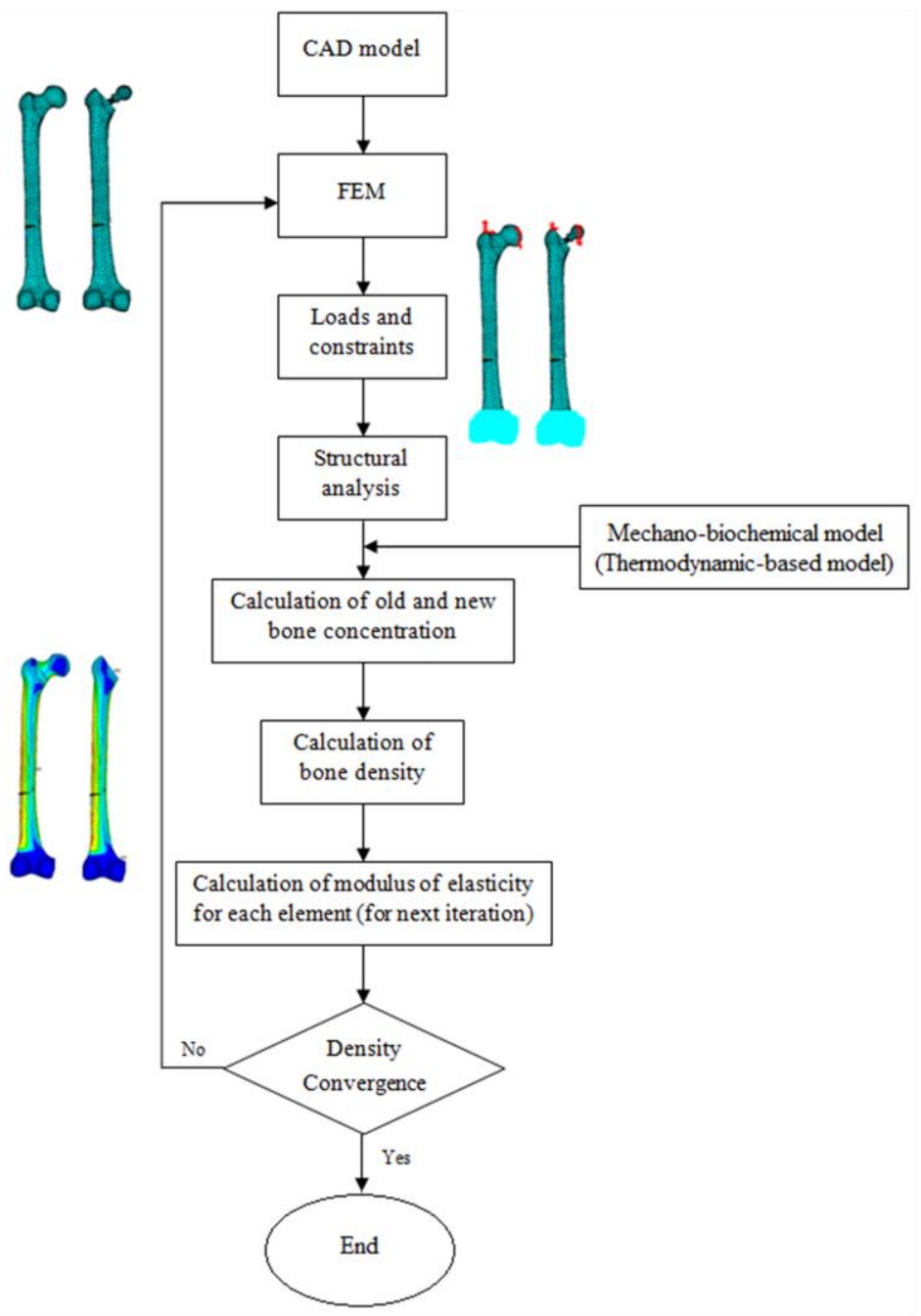

Fig. 6.14: Iterative process of the thermodynamic-based model (mechano-biochemical model) for bone remodeling simulation.

To simulate bone remodeling in response to THA, the elements of the head (and neck) of the intact femur were removed (unselected). The material properties of the hip implant (CoCrMo, Ti6Al4V or CF/PA12 for composite lamina) were assigned to it and the initial material properties of each element of immediate post-operative femur were chosen from the same element in the post convergence-intact femur (inhomogeneous femur). The joint reaction force was then transferred to the hip implant. Finally, the mechano-biochemical model was again applied to simulate bone response to THA and Eq. (6-1) was used to control the convergence. 
In order to simulate bone remodeling due to the presence of a bone plate, the elements of the plate (and screws) with the material properties of $316 \mathrm{~L}$ medical grade stainless steel were added to the model. Moreover, the initial material properties of each element of the femur were chosen from the same element in the post convergence-hip implanted femur. Finally, the mechano-biochemical model was applied and the convergence was controlled to obtain the long-term bone density distribution after hip implantation and plating.

To predict the response of bone to the long-term presence of IM nail, the corresponding material properties (Ti6Al4V or CF/Epoxy for composite lamina) were assigned to the nail. The initial material properties of each element of the immediate post-operative femur were chosen from the same element in the post convergence-intact femur (inhomogeneous femur). Then, the numerical model was applied and Eq. (6-1) was used to control the convergence.

It should be mentioned that in the study by [84], $4^{20}$ possible layups (with different stacking sequence and thickness) for composite IM nail made up of CF/Epoxy was examined to find the optimized configuration which minimize the axial stiffness while maximizing the torsional stiffness for a given range of bending stiffness. In the current study, the femurs implanted with the best configurations ( $\mathrm{C} 1$ to $\mathrm{C} 7$ : Table 1) was investigated in terms of bone remodeling response and compared with Ti6Al4V nailed femur. 


\section{CHAPTER 7 : RESULTS AND DISCUSSION}

\subsection{Bone density convergence}

The iterative process of bone remodeling simulations was considered converged when no significant change in the density of the elements is observed. The convergence of bone remodeling simulations for the intact, conventional hip implanted, unconventional hip implanted, lateral plated and anterior plated femurs as well as metallic IM nailed femur is shown in Fig. 7.1 indicating that the average of density changes over all elements has approached zero after approximately 30-50 iterations.

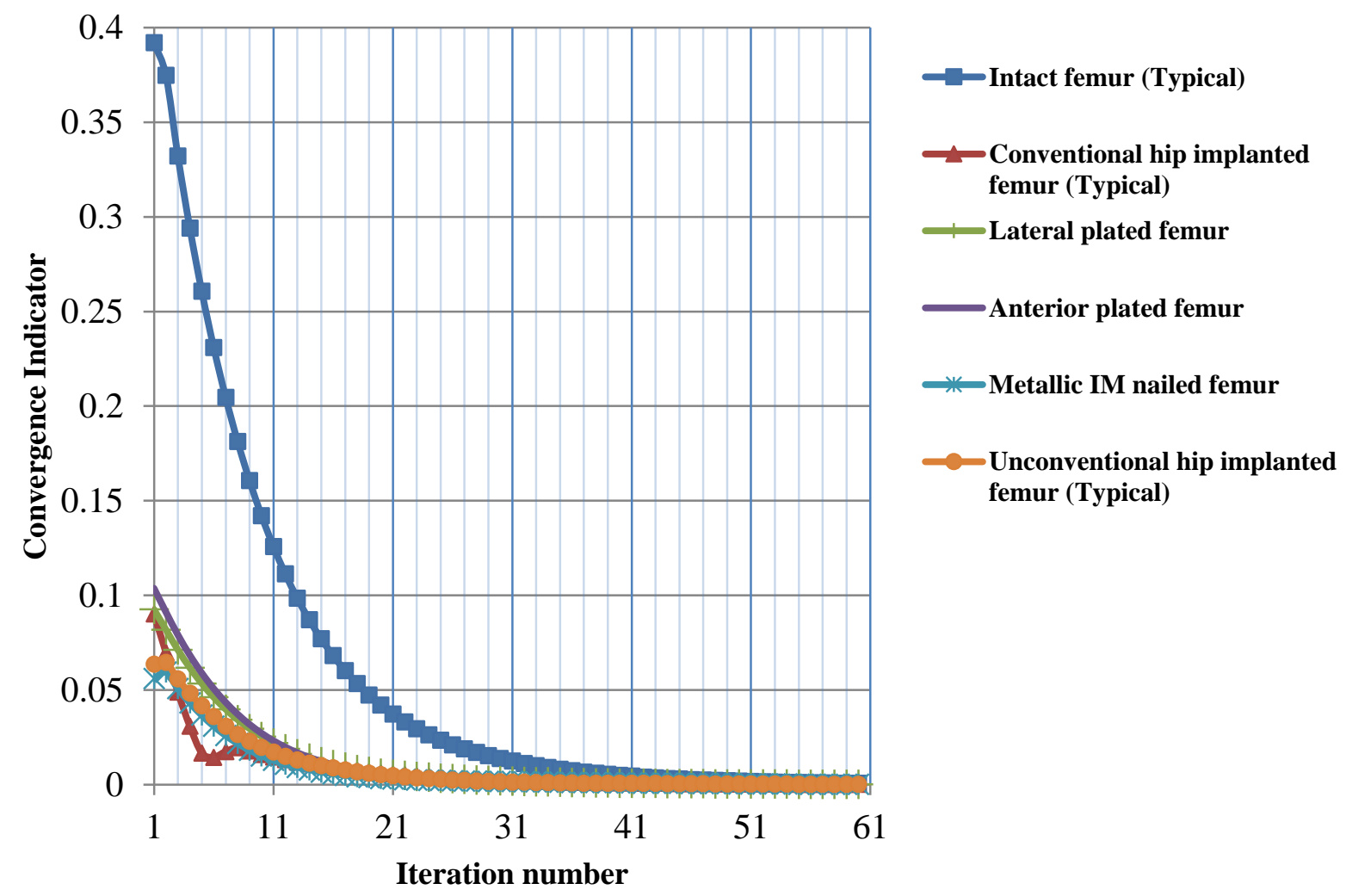

Fig. 7.1: Convergence of bone remodeling simulations. 


\subsection{Distribution of bone density in the intact femur (typical)}

Fig. 7.2 illustrates the intact femur density distribution obtained by the mechano-biochemical model. The bone density throughout the intact femur was found to be in the range of 0.33 to 1.63 $\mathrm{g} / \mathrm{cm}^{3}$ which agrees with the clinical observations $[95,96]$.

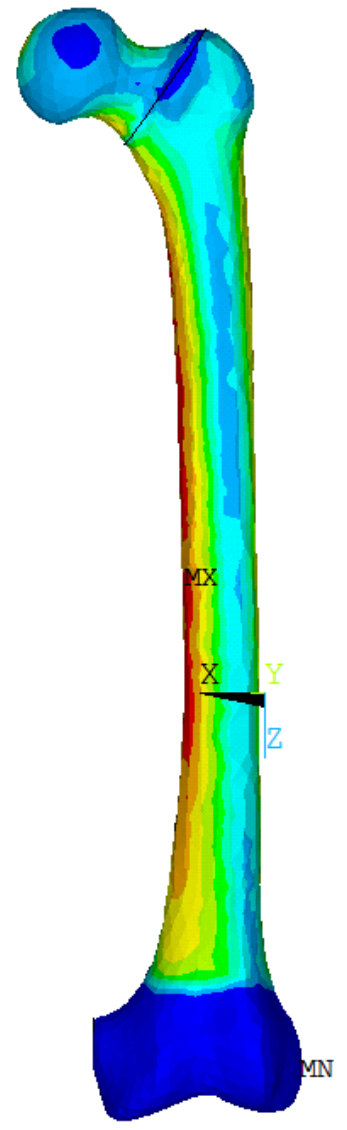

(a)

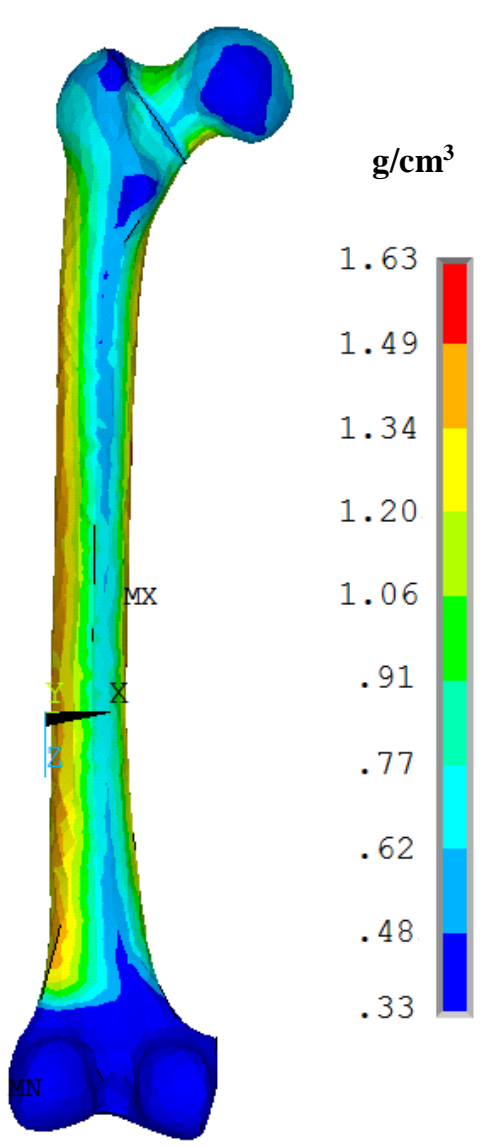

(b)

Fig. 7.2: Density distribution of the intact femur $\left(\mathrm{g} / \mathrm{cm}^{3}\right)$ : (a) anterior view, (b) posterior view.

Furthermore, the density distribution in the intact femur is comparable with the X-ray results obtained by [97] as depicted in Fig. 7.3. In both cases, in addition to the formation of dense cortical bone around the medullary canal, a dense cancellous bone was observed between the calcar and the location of hip joint reaction force. Similarly, such a dense trabeculae carrying the stress from 
the superior contact surface to the calcar region of the medial cortex was seen in the computational study by [5]. Moreover, a dense cortical bone in both proximal and distal regions of the femoral neck was observed, alike to the X-ray results.

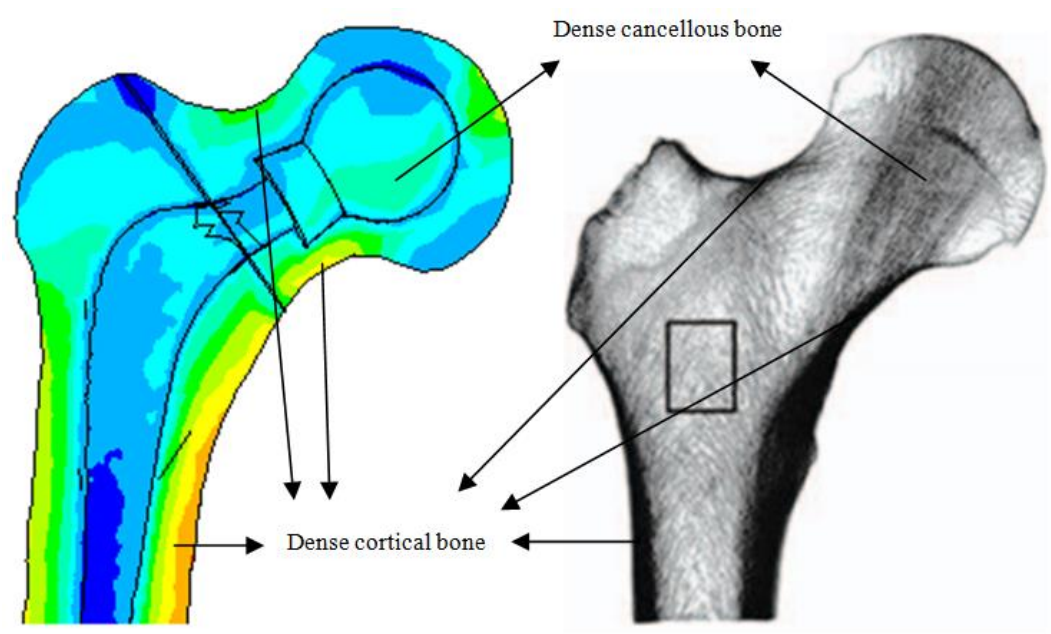

(a)

(b)

Fig. 7.3: Density distribution of the intact femur in the coronal section: obtained by (a) mechano-biochemical model, (b) X-ray (Reprinted with permission, from [97]).

\subsection{Bone response to conventional hip implant}

\subsubsection{Post-operative bone density distribution}

Post-operative bone density distribution in response to the conventional hip implant (CoCrMo) is shown in Fig. 7.4. The range of predicted density throughout the post-operative femur was 0.33 $1.70 \mathrm{~g} / \mathrm{cm}^{3}$. The location of maximum density $\left(1.70 \mathrm{~g} / \mathrm{cm}^{3}\right)$ was observed at a point adjacent to the implant in the oblique surface of the proximal femur. This can be explained by the presence of stress concentration due to the proximal load transfer to the femur from the implant. To examine the density distribution in detail, the femur was divided into seven zones commonly known as Gruen zones [98]. These seven zones describe mainly the regions surrounding the proximal part of femur and are clinically used to assess the outcome of the hip replacement surgery. 


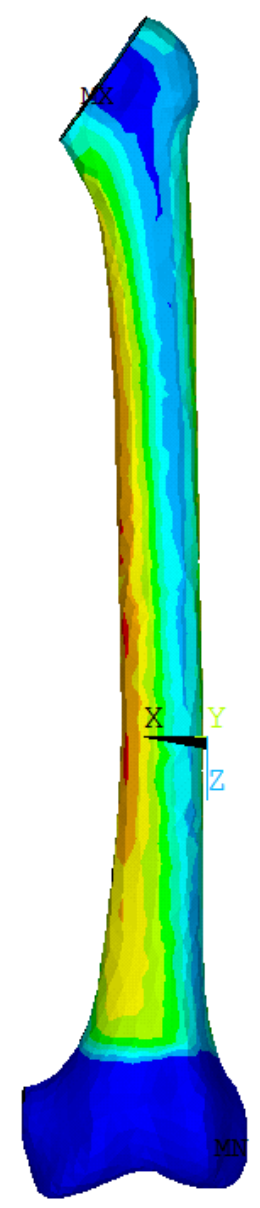

(a)

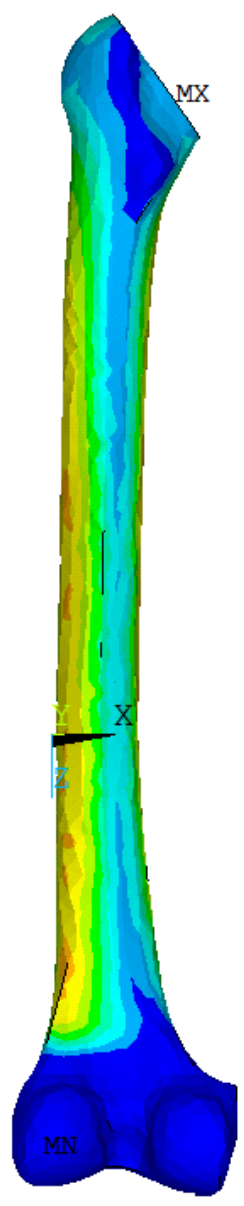

(b)

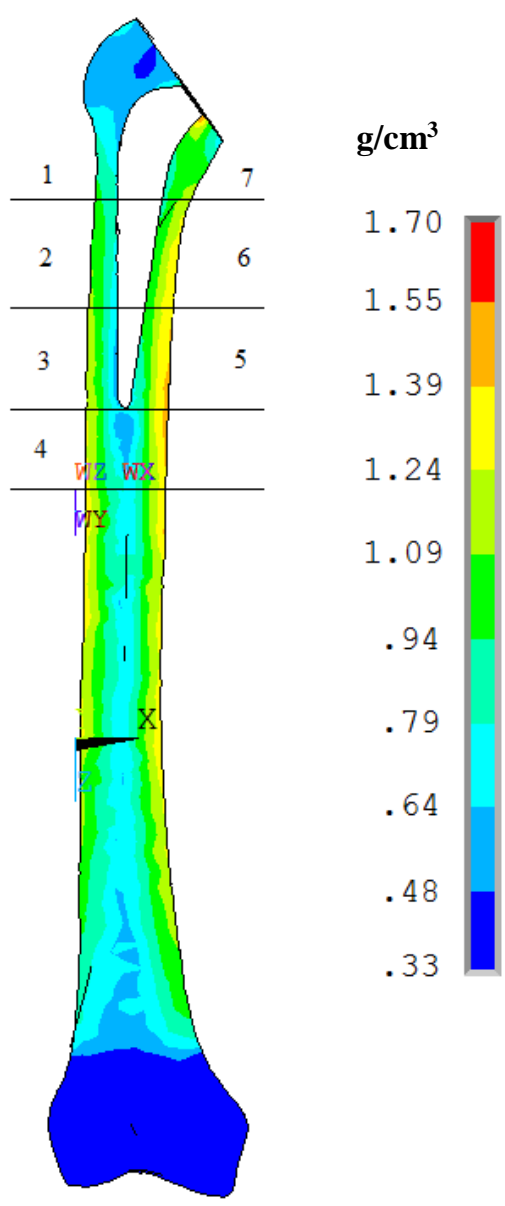

(c)

Fig. 7.4: Post-operative bone density distribution in response to conventional hip implant $\left(\mathrm{g} / \mathrm{cm}^{3}\right)$ : (a) anterior view, (b) posterior view, (c) coronal section of posterior view.

The density evolution of zone 7 in response to THA is illustrated in Fig. 7.5. It is observed that BMD in zone 7 decreased dramatically until iteration 11, then started to increase and eventually after $9 \%$ recovery, it reached a stable condition with the density of $0.90 \mathrm{~g} / \mathrm{cm}^{3}$. This pattern agrees well with Stukenborg-Colsman's observation [28] reporting that after a progressive bone loss in zone 7 during the first six months post-operation, BMD increases and recovers slightly by $7 \%$ at the end of the first year. Therefore, this study suggests that the periprosthetic bone loss is not necessarily progressive and even restoration of bone density can occur, analogous to the 
observations by [99, 100]. In addition, Kroger et al. [12] reported that after a rapid bone loss in response to THA, BMD gets stable which agrees with the present findings.

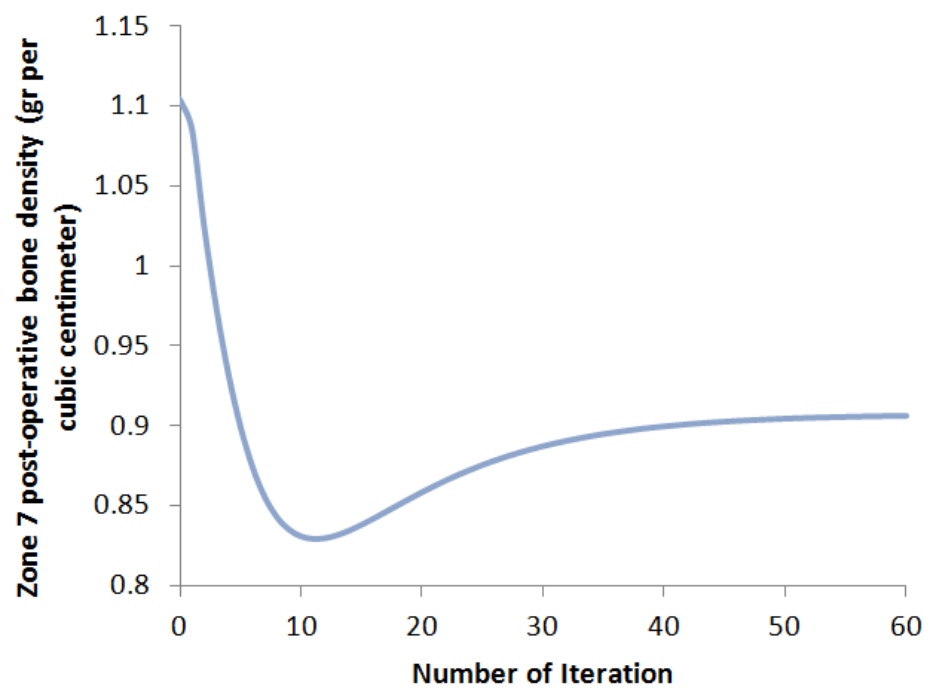

Fig. 7.5: Graph showing the post-operative bone density vs. number of iteration for zone 7 .

\subsubsection{Percent change in femoral density}

Percent change in the femoral density in response to THA with conventional hip implant is presented in Fig. 7.6. The maximum periprosthetic bone loss predicted by the model was $-46.25 \%$ observed in the calcar, which is consistent with Niinimaki and Jalovaara s' observation [101] reporting the maximum bone loss of $-40 \%$ in the calcar area. The $6 \%$ discrepancy between the results can be justified by the difference between the implants stiffness (CoCrMo vs. Steel); the more flexible the implant is, the less the effect of stress shielding will be.

According to our study, the maximum bone formation of $+128.12 \%$ was seen in a location in the oblique surface of the proximal femur adjacent to the implant. The high level of stress transferred to the proximal femur from the implant could result in such a bone densification which in turn may increase the risk of fracture in the femoral neck. 


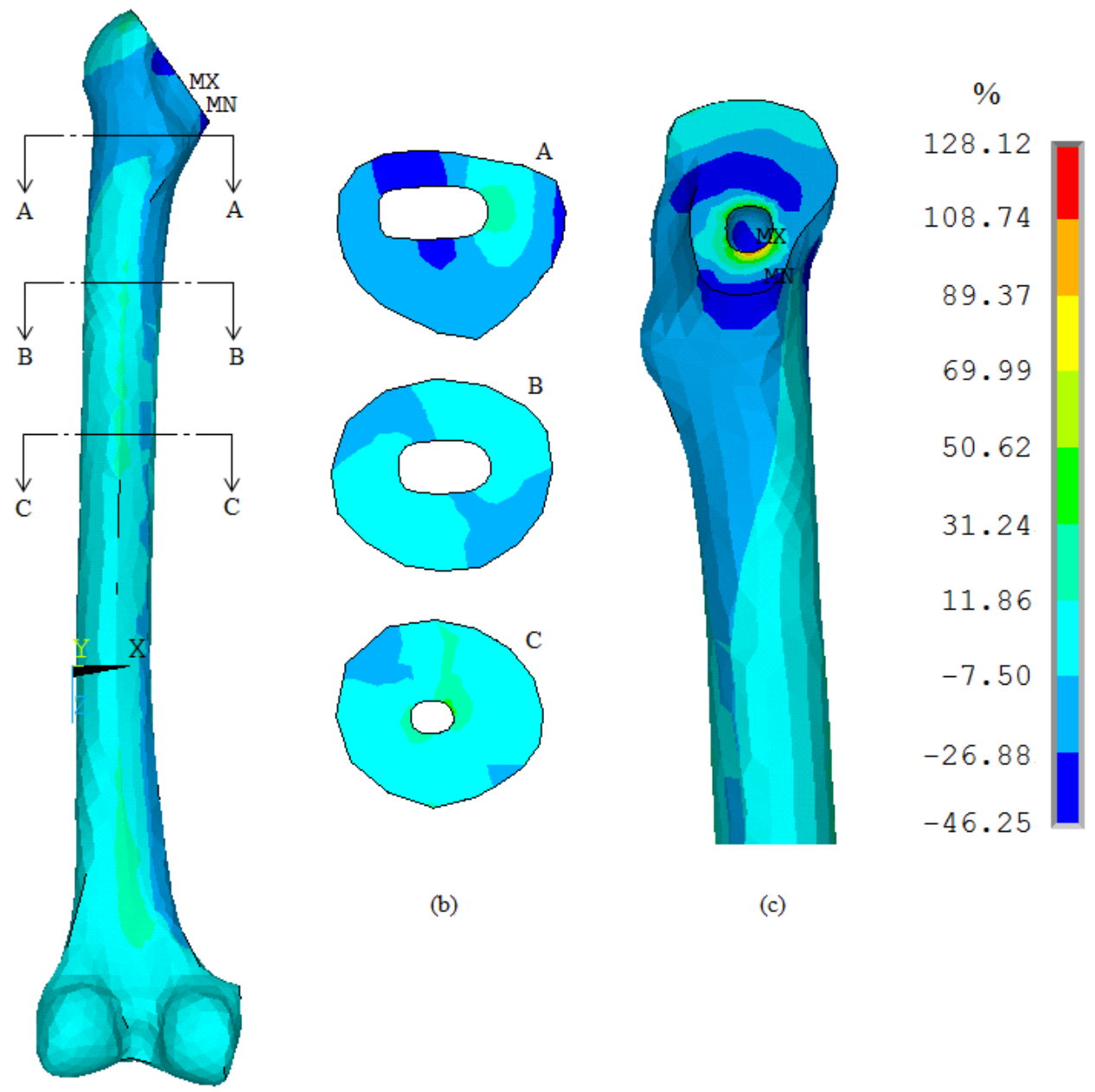

(a)

Fig. 7.6: Percent change in bone density due to the long-term presence of conventional hip implant: (a) posterior view, (b) three transverse segments, (c) medial view.

A-A segment in Fig. 7.6 illustrates cortical resorption as well as trabecular densification at the medial edge of the implant, similar to [14]. The results show that the periprosthetic bone loss decreased as the segment plane moved distally from the proximal metaphysis to mid-diaphysis such that the dramatic range of bone loss $(-46.25 \%$ to $-26.88 \%)$ vanished in B-B and C-C segments, and even some bone densification occurred in the area adjacent to the distal tip of the stem. Also, in A-A segment, it is qualitatively visible that the bone density loss in the posterior region of the proximal metaphysis was greater than that in the anterior side, analogous to Shim's observation 
[102] reporting the posterior region of the metaphysis gets more unloaded compared to the anterior side after THA.

The changes (pre- vs. post-operation) in average density over the elements of each Gruen zone are shown in Fig. 7.7. Among Gruen zones, the maximum periprosthetic bone loss occurred in zone 7 followed by zone 1 with the percentage of $-17.93 \%$ and $-13.77 \%$, respectively. Similarly, in Stukenborg-Colsman's clinical examination [28], using a Ti alloy implant, the strongest decreases in BMD were observed in zone $7(-12 \%)$ and zone $1(-11 \%)$.

In the study by [16] who simulated bone remodeling in response to CoCr implant (Epoch), a maximum bone loss of $-10 \%$ (zone 7) was predicted after 2 years post-operation. In terms of the location (zone) of maximum bone loss, our findings are consistent with [16]. However, there is an $8 \%$ difference quantitatively. With that in mind, our result (-17.93\%) was closer to the clinical value (-16\%) used as a touchstone in their study. Therefore, it may be concluded that the mechanobiochemical model can more realistically predict the maximum bone loss in response to THA. Fig. 7.7 also shows that bone densification only occurred in zone $4(+4.63 \%)$. This is due to the fact that at the distal tip of the stem, all forces are transferred to the bone; this results in a high stress concentration which consequently induces a prominent deposition of bone mass at this zone.

It should be noted that, according to the results, the maximum local bone loss $(-46.25 \%$ in the most proximal/medial area of the femur) is even more than twice the maximum average bone loss ($17.93 \%$ in zone 7). That being said, mechano-biochemical model warns that the average bone loss in the Gruen zones which is mainly used as a touchstone to report the periprosthetic bone loss might be unsuitable since some locations in the bone with a huge bone loss may exist that may result in unpredictable local failure. 


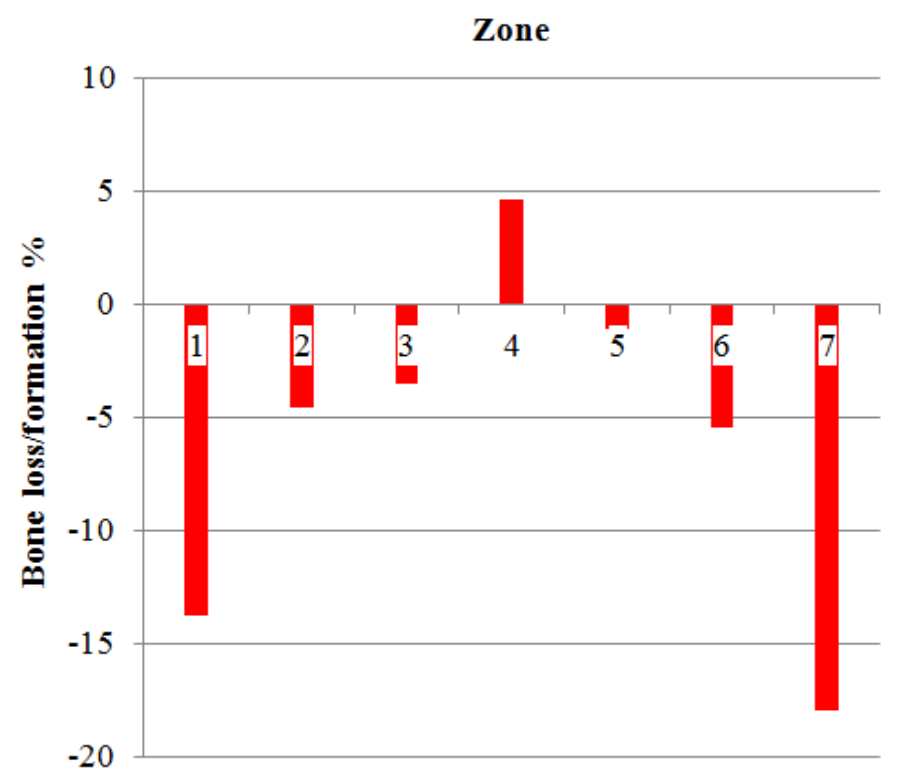

Fig. 7.7: Percentage of bone loss/formation in Gruen zones due to the long-term presence of conventional hip implant.

\subsubsection{Validation of simulations}

In order to validate the simulations against clinical studies, the results were compared to the study by $\mathrm{Li}$ et al. [25] who measured the periprosthetic changes in BMD in response to THA using DEXA. [25] reported that 2 years after THA with a CoCr implant, the maximum bone loss occurs in zone 7 with a percent change of $-19.7 \%$ which is consistent with our results (zone 7: -17.93\%). In zones 2 and 6, our model under-estimated the bone loss (while it still agrees with Glassman's study reporting density changes of $-3.24 \%$ and $-7.72 \%$ for zones 2 and 6 , respectively [103]) compared to the Li's observation. However, for the rest of the regions, our results are close to the clinical ones (Fig. 7.8 : $-13.77 \%$ vs. $-9.2 \%$ for zone $1 ;-3.48 \%$ vs. $-3.2 \%$ for zone $3 ;+4.63 \%$ vs. $+3.4 \%$ for zone $4 ;-1.11 \%$ vs. $-0.9 \%$ for zone $5 ;-17.93 \%$ vs. $-19.7 \%$ for zone 7 ).

The present study also shows that the bone loss in the Gruen zones progressively declined as the region of interest moved distally from the proximal metaphysis to mid-diaphysis which agrees 
with Li's observations. Furthermore, Li's study confirms our results stating that among Gruen zones, zone 4 is the only region showing increase in BMD [25].

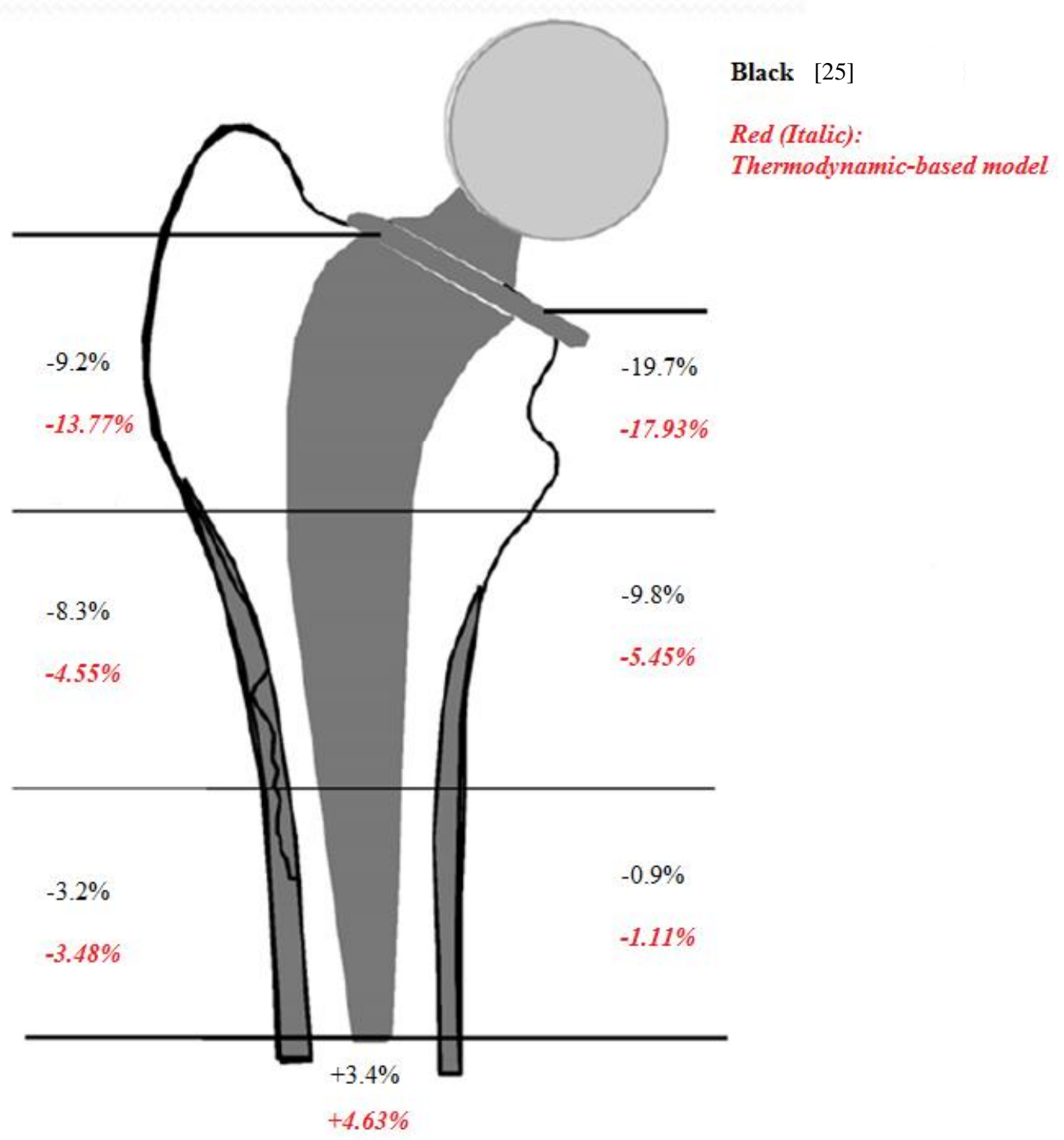

Fig. 7.8: Periprosthetic bone loss observed by [25] (Black values) compared to with that obtained by the mechanobiochemical model (Red Italic values). 


\subsection{Bone response to unconventional hip implants}

\subsubsection{Post-operative bone density distribution}

Periprosthetic bone density distribution in response to three unconventional hip implants (CoCrMo, Ti6A14V and CF/PA12 developed by [9]) is illustrated in Fig. 7.9. Similar to the study by [13], periprosthetic bone loss in response to metallic implants was more severe than that in the presence of composite hip stem as low density regions $\left(0.34-0.8 \mathrm{~g} / \mathrm{cm}^{3}\right)$ were dominant in the femur implanted with CoCrMo compared to that with the composite hip stem. This can be explained by the fact that stiff implants carry a greater portion of the applied load compared to flexible ones. Subsequently, according to the load sharing rule, less load portion is borne by a femur with a stiff implant compared to the one implanted with a flexible implant. On the other hand, based on Wolff's law [20] and Frost's mechanostat theory [104], bone remodeling is stimulated by the local mechanical elastic deformation of bone; thus, bone adapts its properties according to the mechanical load acting upon it. According to these theories, reduction in the mechanical load causes bone to adapt itself by reducing its density. That is why a femur implanted with a stiff implant is subjected to more severe bone loss compared to a femur with a flexible one as the former carries less mechanical load. Therefore, the results of simulations reveal that biomimetic composite hip stem promotes proximal load transfer to the femur compared to the metallic ones, and hence reduces the amount of bone resorption. 


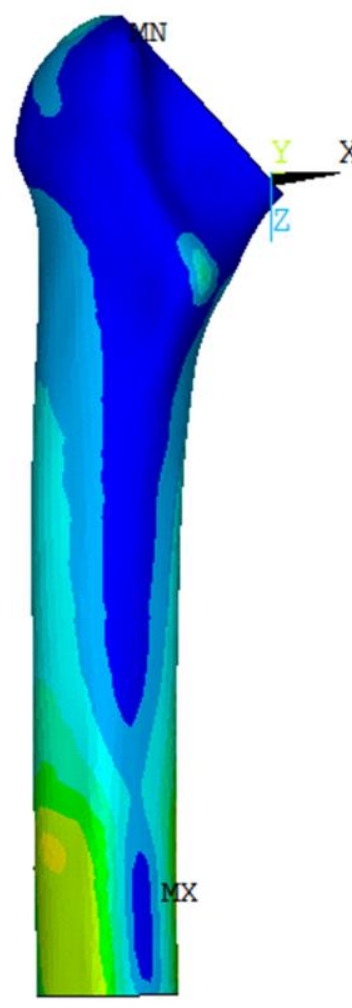

(a)

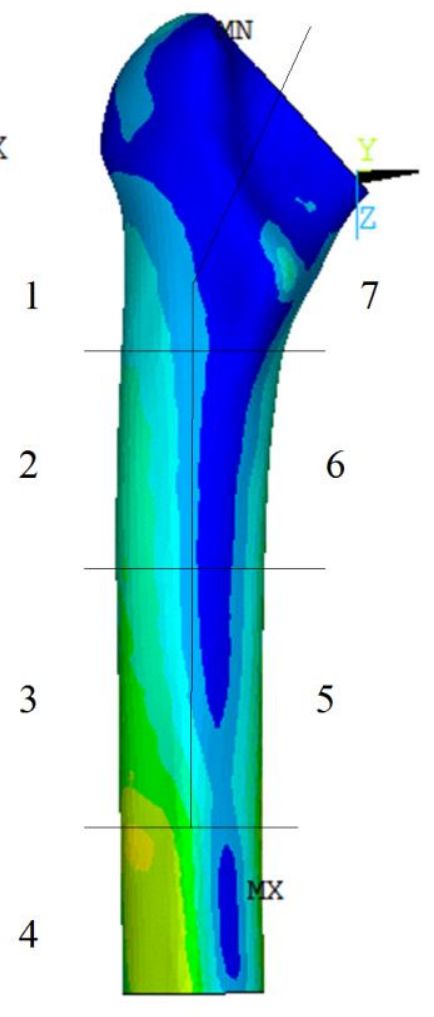

(b)

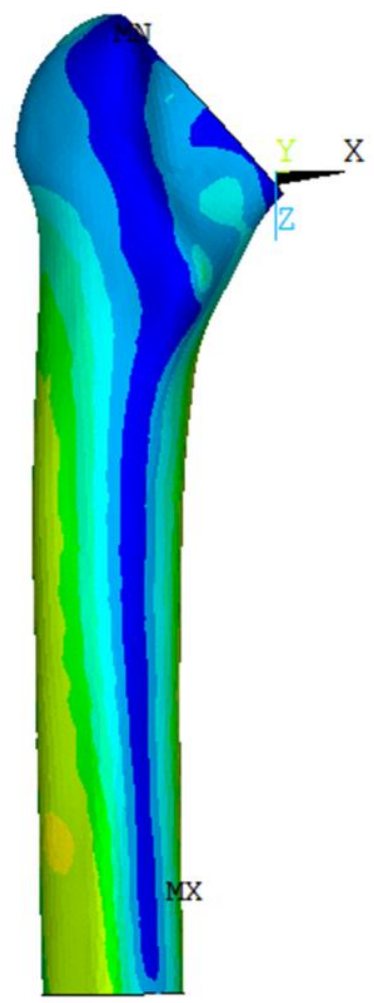

$\mathrm{gr} / \mathrm{cm}^{3}$

.34

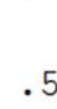

.65

.8

.95

1.1

1.25

1.4

1.55

(c)

Fig. 7.9: Post-operative bone density distribution in response to (a) CoCrMo, (b) Ti6Al4V, (c) CF/PA12 unconventional hip implants.

\subsubsection{Percent change in femoral density}

Percent change in the femoral density in response to the implantation of unconventional hip stems is presented in Fig. 7.10. According to this figure, in all three simulations, the most severe periprosthetic bone loss $(\sim-72 \%)$ occurred in the calcar region which is in agreement with the observation by [105]. Also, the maximum local bone formation $(\sim+60 \%)$ was formed in a region located at the vicinity of the distal tip of the stem. Similarly, [12] found bone mineral density increase in the regions close to the distal implant. This can be justified by the fact that loads sustained by the implant are transferred to the bone through the distal tip of the stem resulting in a stress concentration which consequently induces a deposition of bone mass. 


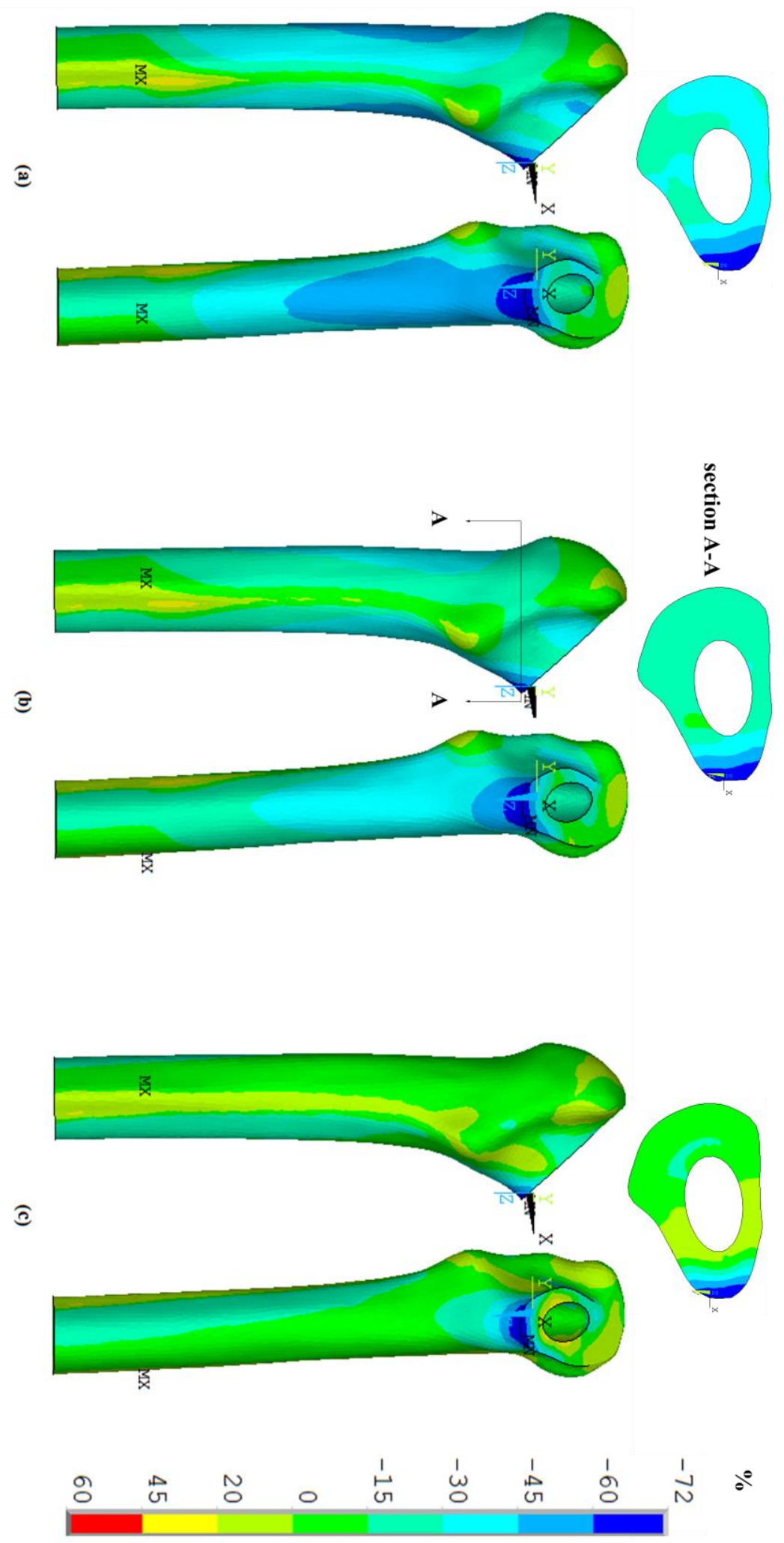

Fig. 7.10: Percent change in bone density due to the long-term presence of (a) CoCrMo, (b) Ti6A14V, (c) CF/PA12 unconventional hip implants. Cutting plane line in all three constructions passes through the proximal metaphysis. 
As illustrated in Fig. 7.10, the stiffness of the implants played a key role in the periprosthetic bone density distribution. It can be observed that only a small portion of the medial femur implanted with composite hip stem was subjected to a severe bone loss (-72\% to $-30 \%)$ unlike the femurs with Ti6Al4V and CoCrMo implants. In addition, section views whose cutting plane passes through the proximal metaphysis depict that a moderately dense trabeculae was developed in the case of composite hip implant only which can boost implant stability; this can be considered as another benefit for the composite hip stem. Our findings also showed an extreme cortical resorption at the medial edge of the implants in the proximal metaphysis analogous to [14].

The percentage of bone loss in Gruen zones is shown in Fig. 7.11 which endorses the advantage of the composite hip stem over metallic ones in terms of induced stress shielding as the femur implanted with composite hip stem showed less total bone loss (-9\%) compared to $-21 \%$ and $-27 \%$ in the presence of Ti6Al4V and CoCrMo, respectively. This figure also shows that the maximum bone loss among Gruen zones were approximately $-43 \%$ (zone 7), -35\% (zone 7), and -10\% (zone 2) in response to CoCrMo, Ti6Al4V and composite hip stems, respectively.

[13] reported that the bone loss due to CF/PA12 stem is approximately between $10 \%$ and $20 \%$ while it lies between 25\% and 50\% when Ti stem is used. In comparison with the results of [13], our findings (total bone loss of $-9 \%$ for CF/PA12 and $-21 \%$ for Ti6AL4V) are close to the lower bands. This can be justified by the inherent difference between the numerical models used for bone remodeling simulations. In contrast to [13] who used the strain energy density model [14, 35] for predicating bone loss, the current study employed the mechano-biochemical model to simulate the response of bone to the presence of composite and metallic implants. The mechano-biochemical model considers the role of biochemical reactions for regulating bone remodeling mechanisms so that in the absence of mechanical loading, the bone resorbs partially by approximately $50 \%[6,8$, 
13] due the presence of biochemical stimulus that triggers bone remodeling. However, in the strain energy density model, bone resorbs completely in the absence of mechanical loading [13].

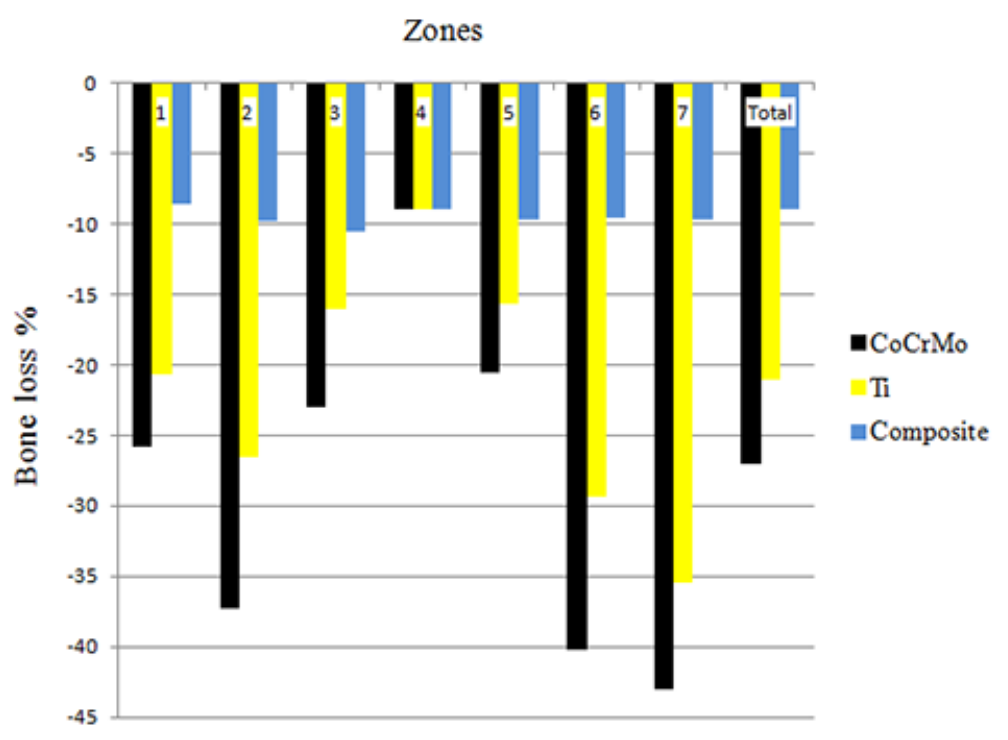

Fig. 7.11: Percentage of bone loss in Gruen zones due to the long-term presence of unconventional hip implants.

In comparison with the clinical study conducted by [106] using a cementless titanium hip implant, the current amount of predicted periprosthetic bone loss in response to Ti6Al4V hip stem, in Gruen zone $7(-35 \%), 6(-29 \%), 4(-9 \%)$, and $1(-20 \%)$ are in good agreement with the range of their observations (zone $7:-40 \%$ to $-10 \%$, zone $6:-30 \%$ to $+1 \%$, zone $4:-9 \%$ to $+4 \%$, zone $1:-36 \%$ to $-12 \%)$. Moreover, our predicted bone loss for all Gruen zones including zone $5(-15 \%), 3(-16 \%)$, and $2(-26 \%)$ are in the range of the results predicted by [5].

In response to the CoCrMo implant (with Young's modulus of $210 \mathrm{GPa}$ ), the maximum predicted bone loss in Gruen zones was $-43 \%$ which occurred in zone 7 and is close to the maximum bone loss of $-40 \%$ (zone 7) in the presence of stainless steel (with Young's modulus of $193 \mathrm{GPa}$ ) observed by [101]. 
The amount of bone loss in response to the composite hip implant was approximately the same ( $-9 \%)$ in all Gruen zones which resulted in a lower standard deviation (0.60\%) compared to the metallic implants $(9.13 \%$ for Ti6Al4V; $12.29 \%$ for CoCrMo). This small deviation implies that the femur implanted with the composite hip implant is not subjected to excessive stress concentration, therefore is under a lower risk of fracture, which can be counted as another privilege of CF/PA12 implant.

In response to the metallic hip implants, the current study showed that the maximum bone loss (43\% for CoCrMo and $-35 \%$ for Ti6Al4V) among Gruen zones occurs in zone 7 similar to other clinical and numerical studies [14, 16, 25, 28, 101, 105, 107-109]. Furthermore, the next most severe periprosthetic bone loss (-40\% for CoCrMo and $-29 \%$ for Ti6Al4V) happened in zone 6 which agrees with the results of [105]. The minimum bone loss (-9\%) among Gruen zones was reported to be in zone 4 (the most distal region) which is consistent with other studies $[14,25,93]$ which reported the minimum bone density decrease occurs in the most distal region of interest. Interestingly, the amount of bone loss in this zone was the same in all three constructions. This can be explained by the fact that since all the loads shared with the implant, regardless of its material, are transmitted to zone 4 , this zone is subjected to the same amount of bone density decrease.

According to our findings, bone loss in response to the metallic implants progressively declined (except for an increase from Gruen zone 1 to 2 ) as the region of interest moves distally from the proximal metaphysis to mid-diaphysis, which agrees with [25]. In addition, our study revealed that the sequence of severity of bone loss (from maximum to minimum) in Gruen zones is as follows: zone $7,6,2,1,3,5$, and 4 for both femurs implanted with CoCrMo and Ti6Al4V which is consistent with the long term observations by [25]. 


\subsection{Bone response to hip implant and bone plate}

\subsubsection{Post-operative bone density distribution}

The iterative process of simulations was continued until no significant change in the density of the elements was observed (after $~ 30$ iterations for plated femurs, Fig. 7.1). From a clinical point of view, this is considered as the long-term response when the femoral density gets to a stable condition. Such a stable condition was also seen in the clinical study by [65] reporting that femoral bone density does not decrease with time after 3 years of the plate application.

Femoral bone density distribution in response to the long-term presence of the hip implant and bone plate (8-hole Zimmer) is illustrated in Fig. 7.12 for both lateral and anterior plated femurs. The maximum density $\left(\sim 1.8 \mathrm{~g} / \mathrm{cm}^{3}\right)$ is observed at the vicinity of the most proximal and distal screw holes for the lateral and anterior plated femurs, respectively. This dense bone formation can be justified by the stress concentration around the screw holes.

\subsubsection{Percent change in femoral density}

Fig. 7.13 demonstrates the percent density changes due to the implantation of hip stem and bone fracture plate for both lateral and anterior plating. The model predicted severe bone loss in the calcar region for both lateral and anterior plated femurs which is due to the presence of hip implant. The prediction of such severe BMD decrease in the calcar region agrees with other studies $[14,16$, $25,28,101,105,107-109]$. On the other hand, intensive bone formation was observed in a region located in the oblique surface of the proximal femur adjacent to the hip implant. The high level of stress transferred to the proximal femur from the implant could result in such a bone densification which may increase the risk of local fracture in the neck of the femur. 
According to Fig. 7.13, the areas directly under the lateral and anterior bone plates experienced severe bone density decrease (up to -70\%) which agrees with the observations by [64] reporting the loss of dense bone in the regions beneath the bone plate. [64] also observed the formation of dense zones adjacent to the proximal and distal ends of the plate as well as around the screws. This is in agreement with our results as some level of bone formation (up to $\sim+110 \%$ ) was predicted around the most proximal and distal screw holes in both lateral and anterior plated femurs.

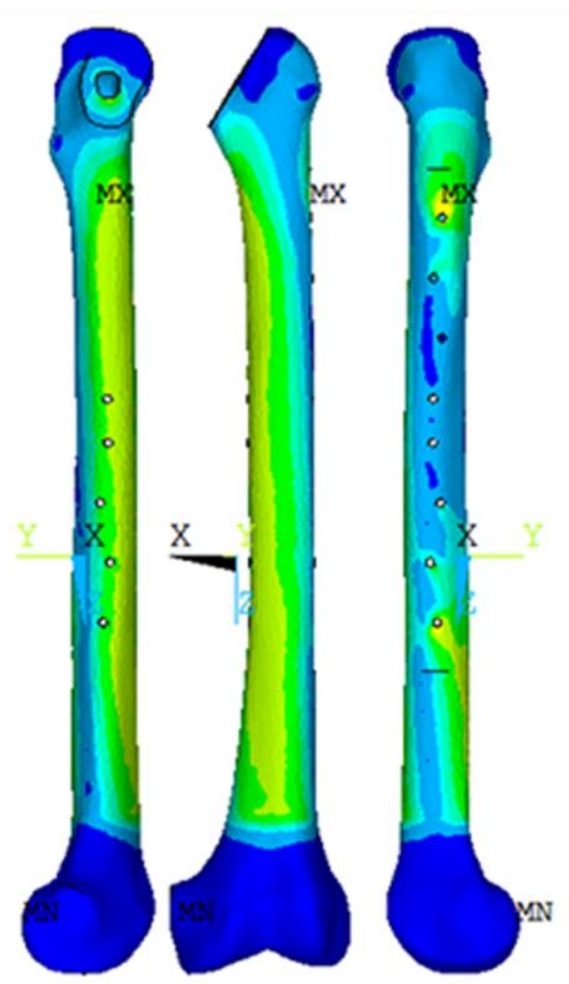

(a)
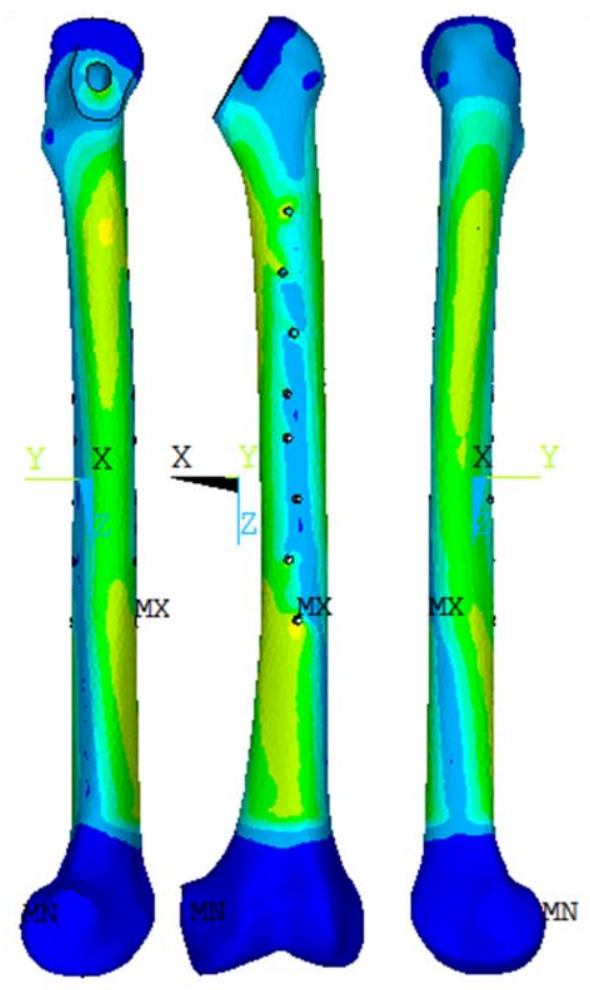

$\mathrm{gr} / \mathrm{cm}^{3}$

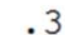

.4

.55

.65

.8

1

1.2

1.5

1.84

Fig. 7.12: Post-operative bone density distribution in response to hip implant and fracture plate: (a) lateral plated femur, (b) anterior plated femur. 


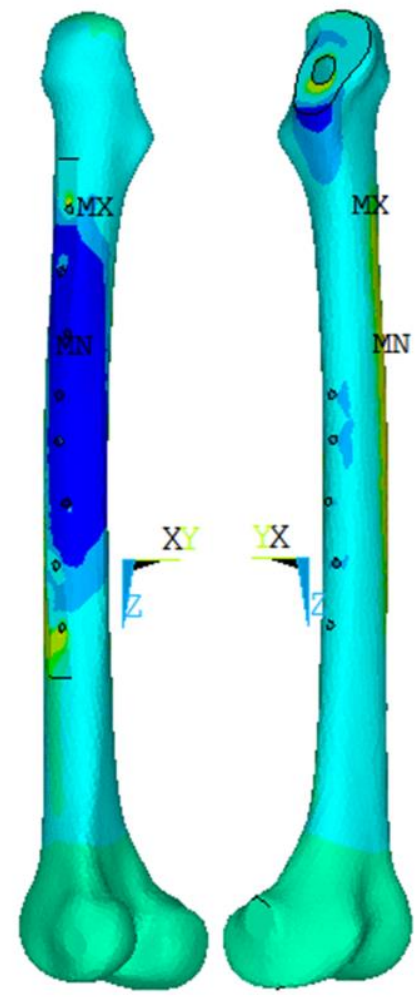

(a)

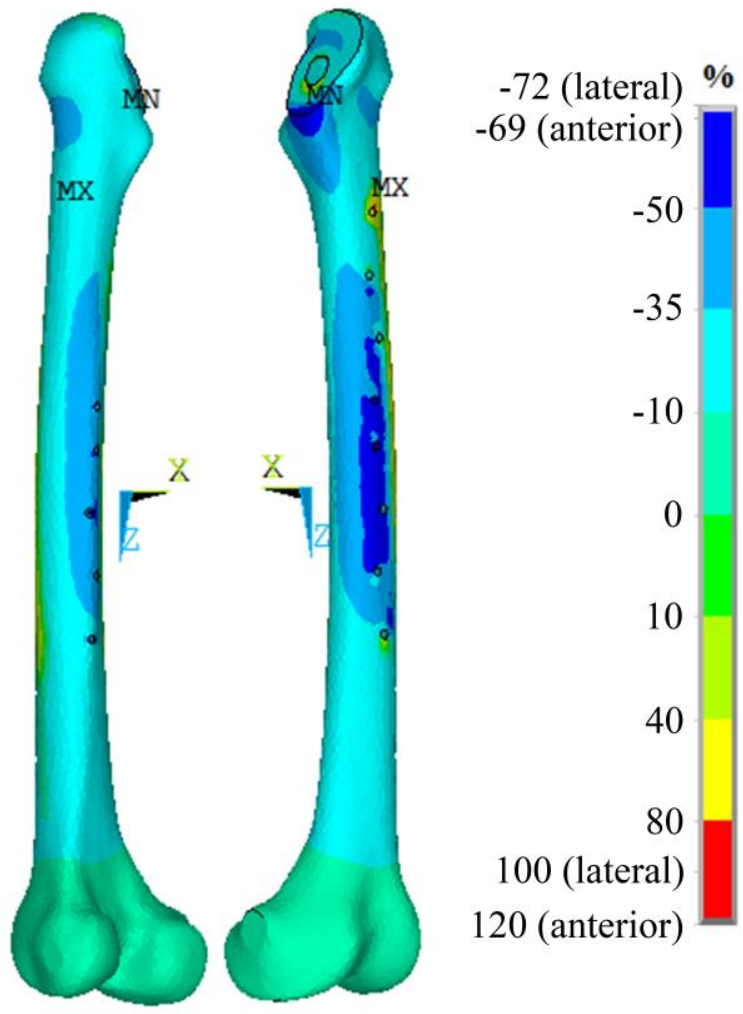

(b)

Fig. 7.13: Percent change in bone density due to the long-term presence of the hip implant and fracture plate: (a) lateral plated femur, (b) anterior plated femur.

For further investigations, the region beneath the plate was divided into six equal length zones as depicted in Fig. 7.14(a). Each zone contains all elements within its volume. In Fig. 7.14(b), bone loss in each zone as well as the average bone loss over all six zones due to the presence of hip implant and bone plate (lateral and anterior plating) is illustrated.

According to Fig. 7.14, the average bone loss was approximately the same for lateral and anterior plated femurs ( -24\%). The figure also reveals that the maximum bone loss in the anterior plated femur occurred in zone 5 while in the lateral plated femur, it was zone 2 that experienced the most severe bone density decrease. [87] who compared the pattern of stress in proximal/lateral regions of intact and lateral plated femurs using infrared thermography camera, stated that the reduction of stress (intact vs. plated femur) in mid-shaft ( equivalent to our zone 2$)$ is higher than that in 
proximal femur ( equivalent to our zone 1). In addition, according to [71], proximal/lateral and distal/lateral regions ( equivalent to our zone 1 and 3, respectively) of lateral plated femur experience higher strain compared to the mid-shaft ( equivalent to our zone 2 ). Hence, those studies [71, 87] justify our results predicting the greater bone loss in zone 2 compared to zone 1 and 3 in response to lateral plating.

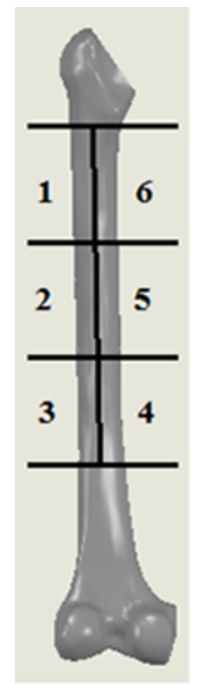

(a)

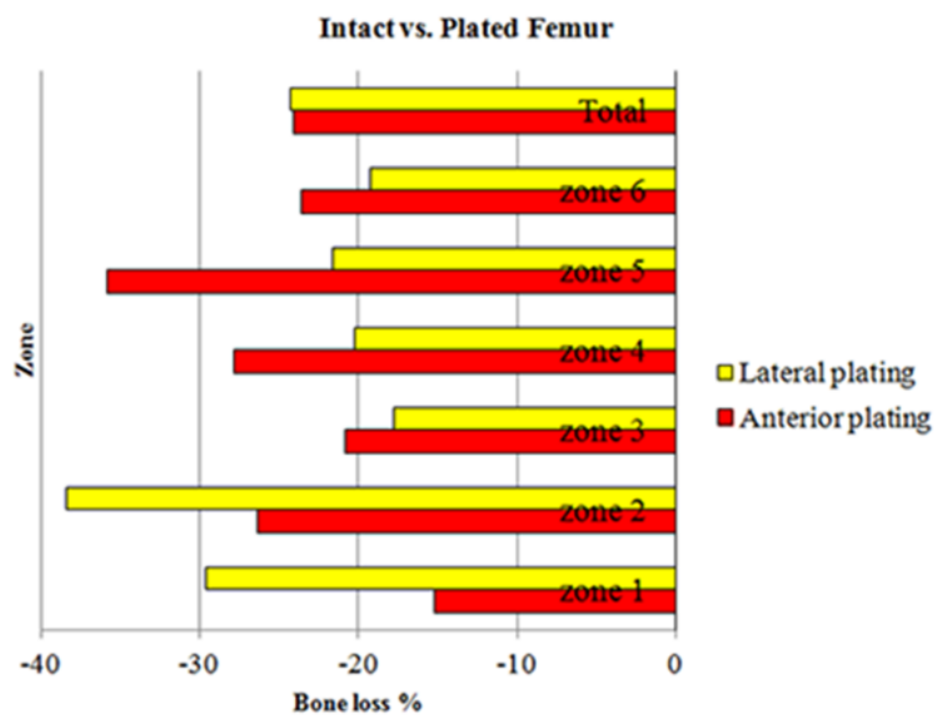

(b)

Fig. 7.14: (a) femoral shaft six zones used to compare bone loss in the regions beneath the plate, (b) percentage of bone loss in six zones due to the long-term presence of hip implant and fracture plate.

Since hip joint and muscle forces acting on the femur tend to medially bend the femoral bone, there is a conventional wisdom believing that femoral bones might be more vulnerable in response to lateral plating rather than anterior plating in terms of bone loss. However, our findings reveal that the average bone loss in response to lateral plating was the same as anterior one $(\sim-24 \%$ in the presence of the hip implant and bone plate). Also, the maximum bone loss among six zones was approximately equivalent for both lateral and anterior plated femurs $(-38 \%$ and $-36 \%$ for lateral and anterior plating, respectively). In addition, the authors came to the conclusion that anterior plating is inferior to the lateral one in terms of the number of zones subjected to severe 
bone loss ( 4 vs. 2 zones) as in zones 3, 4, 5, and 6 of anterior plated femur, the magnitude of bone loss was greater compared to the corresponding zones in the lateral plated femur.

\subsubsection{Validation of simulations}

The mechano-biochemical model is validated in section 7.3 where femoral density change in response to THA (with conventional hip implant) was predicted and compared with clinical trial data. However, to the best of author's knowledge, there is no data regarding long term femoral density changes in response to both hip implant and bone plate in the literature. To validate the results of the present study against clinical trials, we separately simulated bone remodeling of an intact model (with no hip implant) in response to the lateral plating to replicate the conditions of a follow up conducted by [65] in which the long-term femoral bone density (1-14 years from application to removal) in patients who underwent lateral bone plating surgery but no hip implantation was investigated. The region of interest was considered between the second and third holes from the distal end of the plate (shown in Fig. 7.15(a)) and divided into four quadrants (posterior, medial, lateral and anterior) same as the clinical study by [65].

The amount of bone loss (Fig. 7.15(b)) predicted by the mechano-biochemical model in three out of four quadrants correlate with the values reported in the aforementioned clinical study. In other words, the bone loss of posterior $(-32 \%)$, lateral $(-27 \%)$ and anterior $(-15 \%)$ quadrants were in the range of their corresponding clinical measurements, i.e. between $-45 \%$ and $-6 \%$ in posterior, between $-28 \%$ and $-5 \%$ in lateral, and between $-37 \%$ and $-1 \%$ for anterior quadrant [65]. 


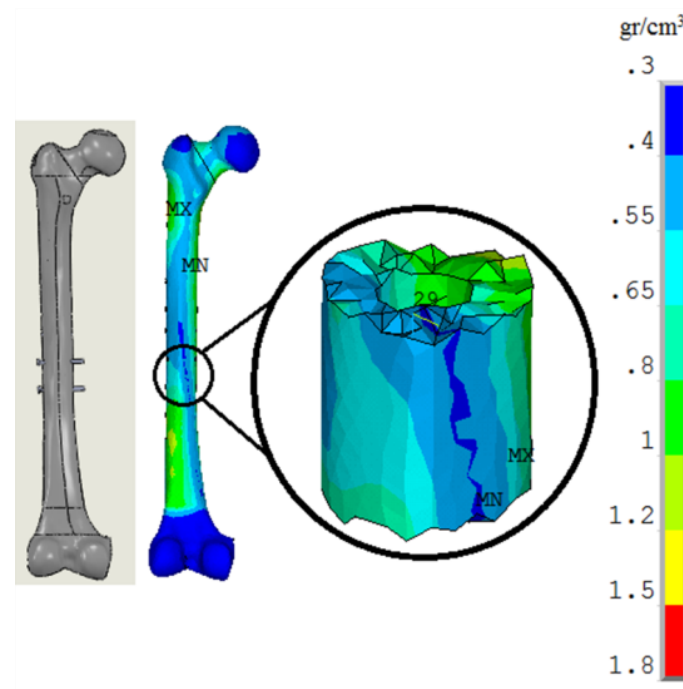

(a)

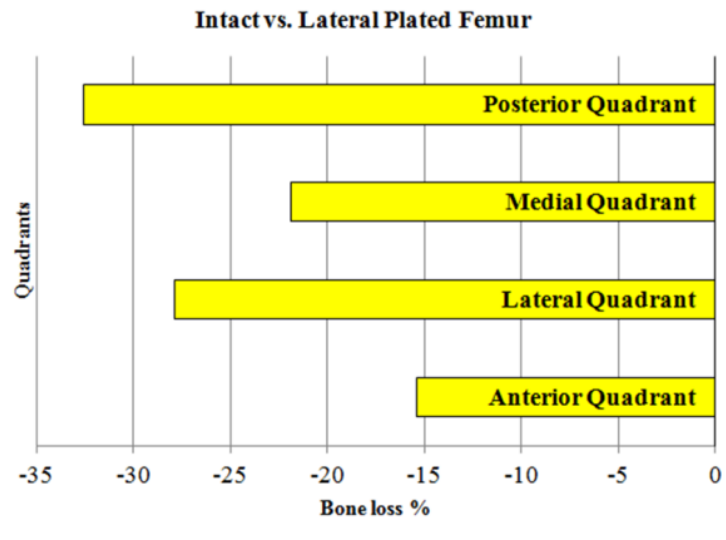

(b)

Fig. 7.15: (a) Selected zone between the second and third hole from the distal end of the plate, (b) bone loss in four quadrants of the selected zone.

\subsection{Bone response to intramedullary nail}

Average bone loss due to the presence of composite nails (CF/Epoxy) with the laminate stacking sequences listed in Table 1 and metallic nail (Stryker T2 nail, made up of Ti6Al4V) is depicted in Fig. 7.16. As expected, among eight configurations, $\mathrm{C} 1$ nail resulted in the lowest bone loss ($2.3 \%$ ) as it has the lowest bending and axial stiffness compared to the other nails while the femur implanted by $\mathrm{C} 8$ (Ti6A14V) nail with the greatest bending and axial stiffness, was subjected to the most severe bone loss $(-7.9 \%)$.

[84] found that the composite IM nail of C5 with an axial stiffness of $4.43 \mathrm{MN}$, bending and torsional stiffness of 80.1 and $73.8 \mathrm{~N} . \mathrm{m}^{2}$ respectively shows an overall superiority compared to the other composite configurations in terms of fracture stability and strength in oblique and transverse shaft fractures. Therefore, the femur implanted with this winning composite candidate (C5) is selected to be compared in detail with Ti6Al4V (C8) nailed femur in terms of bone 
remodeling response. Fig. 7.17 illustrates the post-operative femoral density while Fig. 7.18 shows the percent change in BMD in response to $\mathrm{C} 5$ and Ti6A14V nails.

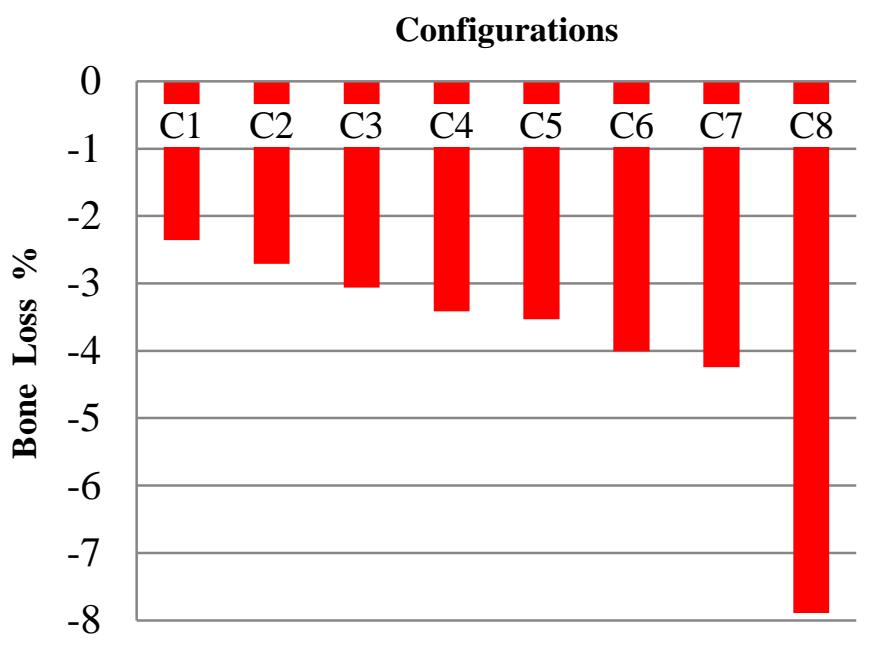

Fig. 7.16: Percentage of bone loss (intact vs. nailed femur) due to the long-term presence of IM nail for eight configurations.

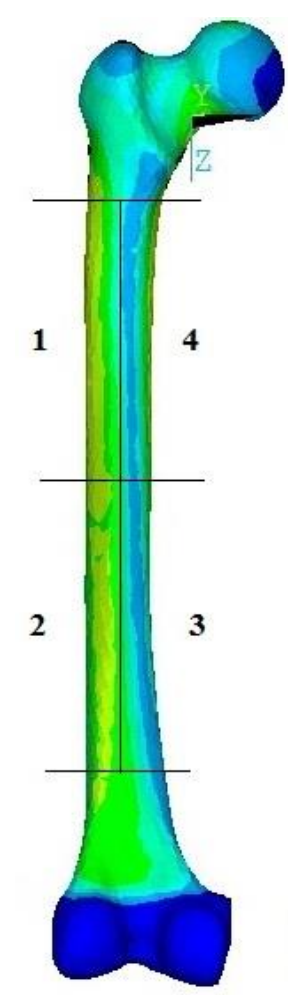

(a)

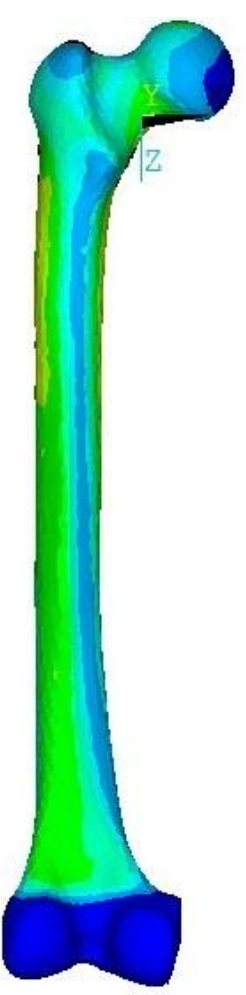

(b)

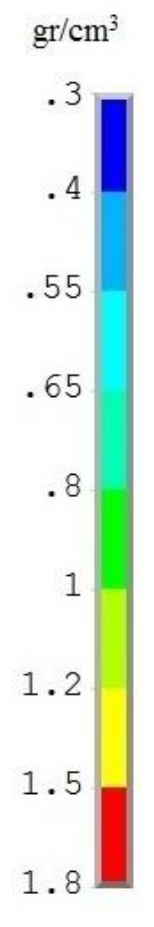

Fig. 7.17: Post-operative bone density distribution in response to (a) C5, (b) Ti6Al4V nails. 
As illustrated in Fig. 7.18, femoral bone experienced some moderate level of bone loss as a result of C5 IM nailing unlike the femur implanted with Ti6Al4V nail so that the range of severe bone loss $(-30 \%$ to $-15 \%)$ vanished in the femur with C5 nail inside. This can be counted as one of the privilege of composite nail over the metallic one. By comparing the long-term femoral density distribution as a result of hip implantation or plating (shown in sections 7.3-7.5) with IM nailed femur, one can conclude that the bone loss severity due to the presence of metallic hip implants or plates is much more than that as a result of metallic IM nailing (up to $\sim-70 \%$ vs. $\sim-30 \%$ bone loss).

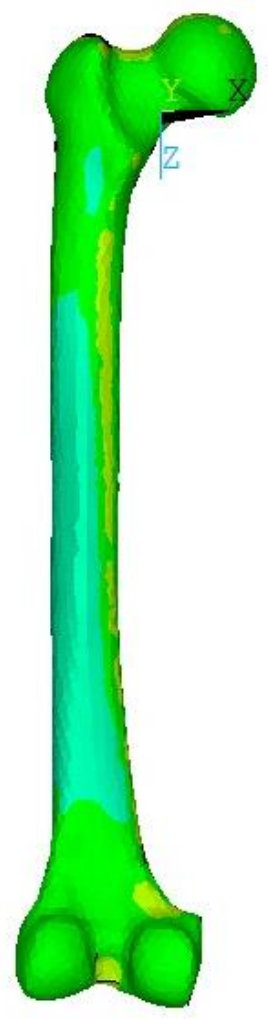

(a)

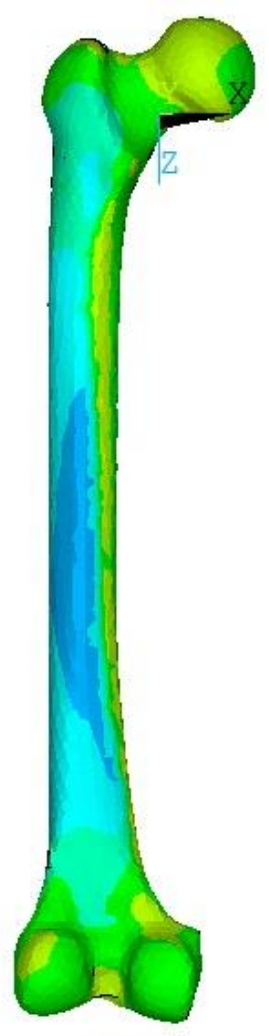

(b)

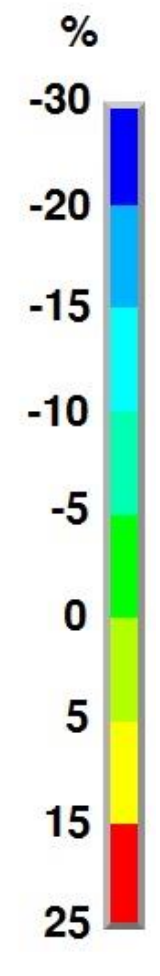

Fig. 7.18: Percent change in bone density due to the long-term presence of (a) C5 nail, (b) Ti6Al4V nail. 


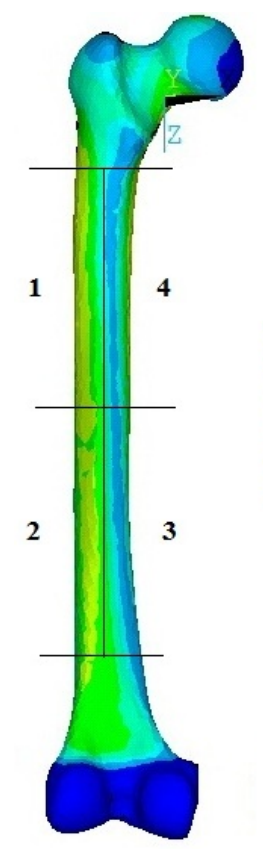

(a)

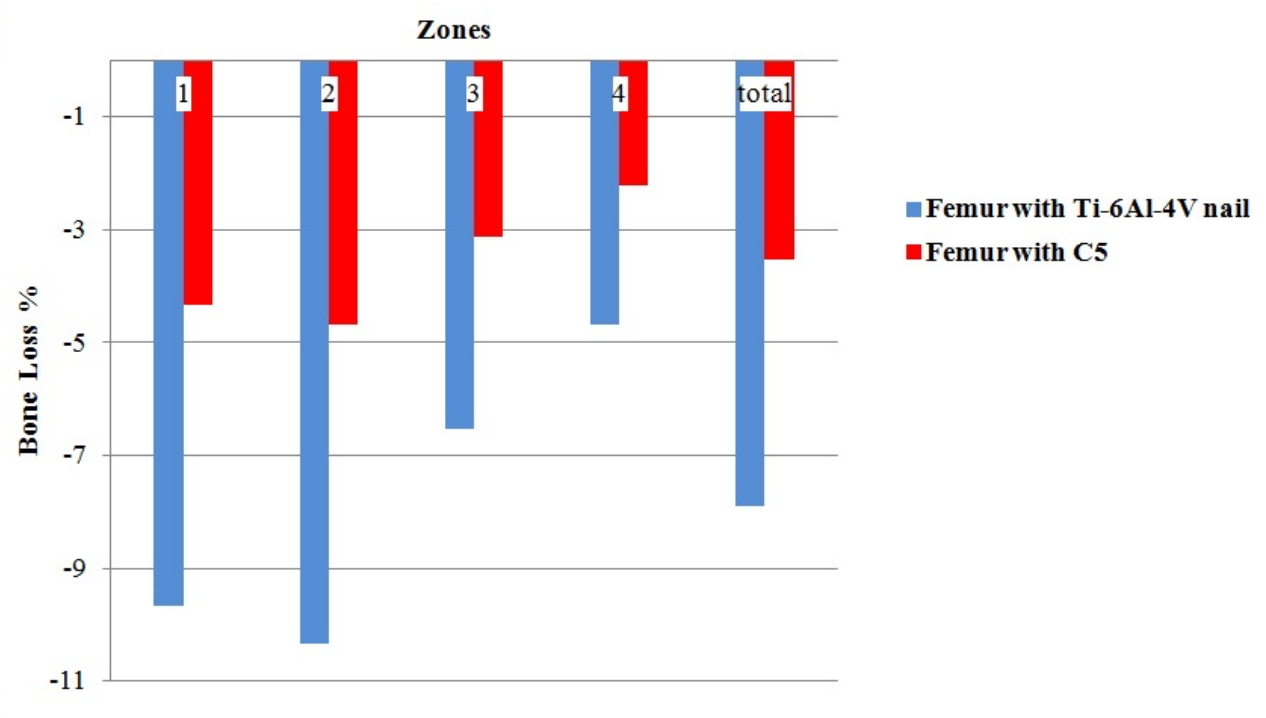

(b)

Fig. 7.19: (a) Selected four zones in the femoral shaft, (b) percentage of bone loss in six zones due to the long-term presence of IM nails.

For further investigations, the implanted femurs were divided into four zones (Fig. 7.19(a)). As depicted in Fig. 7.19(b), among four zones, zone 2 was subjected to the maximum bone loss ($4.7 \%$ and $-10.3 \%$ in the presence of C5 and Ti6Al4V nails, respectively) while zone 4 experienced the lowest bone density decrease $(-2.2 \%$ and $-4.7 \%$ in the presence of $\mathrm{C} 5$ and Ti6Al4V nails, respectively). Examining the figure, one can observe that the implantation of $\mathrm{C} 5$ composite nail resulted in the total bone loss of $-3.5 \%$ while Ti6Al4V nail increased the bone loss to $-7.9 \%$ which is in the range of Wang et al.'s results (-9\% to -1\% bone loss in the femoral shaft) [110]. In addition, predicted BMD decrease in response to Ti6Al4V nail in zone 1 and 2 were $-9.7 \%$ and $-10.3 \%$, respectively which are close to the range of $-9.1 \%$ to $-3.9 \%$ bone loss reported for the lateral side of Ti nailed femur [110]. Furthermore, predicted bone loss in zone 3 and 4 were $-6.5 \%$ and $-4.7 \%$, respectively which lie between $-6.5 \%$ and $-0.3 \%$ bone loss reported for the medial side of the femur [110]. 
In a clinical study by [111], it is reported that in response to the long-term (29 months) presence of an IM nail, BMD in human tibia treated by retained reamed IM nail decreases by $7 \%$ compared to that in the intact contra-lateral one which is consistent with our results (-7.9\%).

Fig. 7.19(b) also reveals that among all four zones, zone 2 experienced the maximum bone loss of $-4.7 \%$ and $-10.3 \%$ in the presence of C5 and Ti6Al4V nails, respectively. It was followed by zone 1 with the density decrease of $-4.3 \%$ and $-9.7 \%$ in response to C5 and Ti6Al4V nails, respectively. In other words, it can be concuded that the lateral side of the femur got more unloaded compared to the medial side after IM nailing which agrees with the results of [110].

Among four zones, the variation of bone loss in response to C5 nail is lower than that in response to Ti6Al4V nail (standard deviation: $1.13 \%$ vs. $2.6 \%$ for $\mathrm{C} 5$ and Ti6Al4V, respectively). This implies that the stress distribution in the femur implanted with the composite nail is more uniform compared to the one with the metallic nail, therefore the femur treated by the composite nail would be under a lower risk of local fracture as it does not experience excessive stress concentration. This can be considered as another advantage of the composite nail over the metallic one. 


\section{CHAPTER 8 : CONCLUSIONS, LIMITATIONS AND FUTURE WORK}

\subsection{Conclusions}

A mechano-biochemical model, which considers the coupling between the mechanical loading and biochemical affinity as stimulus for bone remodeling, was employed in this study to simulate the long-term behaviour of the femur in response to total hip arthroplasty, plating and intramedullary nailing. This study provided a quantitative figure for monitoring bone density changes throughout the femoral bone. The current research revealed that:

- $\quad$ The most significant periprosthetic bone loss in response to THA occurred in Gruen zone 7 (up to $-43 \%$ in response to unconventional hip implant/CoCrMo). Conversely, zone 4 experienced the lowest bone mineral density (BMD) decrease among Gruen zones.

- $\quad$ The composite hip implant (CF/PA12) was more advantageous over the metallic ones (made up of Ti6Al4V and CoCrMo) as it induced less stress shielding (total bone loss of $-9 \%$ compared to $-21 \%$ and $-27 \%$ in the presence of Ti6Al4V and CoCrMo implants, respectively), developed a moderate dense trabeculae at the vicinity of the implant, and provided more uniform bone density change following THA.

- $\quad$ The areas directly beneath the bone fracture plate experienced severe bone loss $(\sim-70 \%)$. However, some level of bone formation was observed at the vicinity of the most proximal and distal screw holes in both lateral and anterior plated femurs (up to $\sim+110 \%$ ). Furthermore, in terms of bone remodeling, the anterior plating was not superior to the lateral plating. 


\subsection{Limitations of the study}

Although the results of research study agree well with the bone morphology and literature, the model has some limitations and simplifications:

In the current study, "no separation" contact type was considered between the fracture plate and bone which allowed for the fracture plate to slide over the bone unlike "bonded" contact type which was assumed between the hip implant and bone. Several studies [71, 87-89] also considered similar assumptions for modeling femoral fracture fixation and total hip arthroplasty. These assumptions are similar to the clinical conditions in which bone remodels itself, and simulate perfect interdigitation of bone around the threads of the screws, and full bony in-growth around the hip implant. Bonded contact type disregards micromotions at the bone-implant interface which, in turn, increases the load applied on the bone. This assumption may underestimate the amount of bone loss predicted by the model. This is similar to the effect of isotropic behaviour assumption on the final bone density distribution since considering isotropic behaviour ( elastic modulus in longitudinal direction) for each element may result in a more rigid bone compared to the bone with anisotropic structure. This increase in rigidity can increase the magnitude of the load carried by the bone and subsequently can reduce the bone loss predicted by the model. In this research study, bone viscoelastic behaviour was ignored in the modeling as it is negligible for the range of physiological strain rate [112]. Furthermore, since the long-term response of femur to the presence of hip implant and bone plate was the focus of this work, healing mechanism was not incorporated into the model, and subsequently callus properties were ignored. The ignorance of callus properties in the simulations can be justified by the facts that the proposed mechanobiochemical model is not sensitive to initial material properties. As a result, even if the callus properties were considered in the simulations, the results (i.e. long-term density distribution of the 
implanted femur fixed with a bone fracture plate) would not get affected. This is due to the fact that callus properties are the initial material properties for the last step of simulations where the long-term response of the implanted femur to the bone fracture plate was of interest. In the current study, the computational steps are unrelated to real time. However, the iterative process was assumed converged when no significant change in the density of the elements was observed, which from a clinical point of view is considered as the long-term response when the femoral density gets to a stable condition.

\subsection{Future work}

In the current work, the long-term response of femoral bone to the presence of hip implant, bone fracture plate and IM nail is simulated. However, orthopedic implantation is not limited to those cases. For example, hip resurfacing arthroplasty and short stem implantation can be explored as a future work. In addition, in the current research study, the bone density distribution

In this study, we tried to bring insight into bone loss due to the stress shielding phenomena, therefore the density decrease caused by purely chemical diseases (e.g. bone tumor) was not considered. It should be mentioned that the proposed model is capable of considering the effect of chemical environments on bone remodeling through the biochemical reaction rates and the concentration of substances participating in the reactions. However, further research is indeed needed to quantify the effect of chemical diseases on the concentration of substances and the rate of biochemical reactions.

In addition, in the thermodynamic-based model, the treatment of the bone remodeling process is based on a coordinated sequence of bone resorption and formation. This coordination is somewhat 
simplistic, and further research is required to extend the model to contain more details about the bone growth control mechanisms involved in the bone remodeling process. 


\section{REFERENCES}

[1] Frost HM. Changing concepts in skeletal physiology: Wolff's law, the mechanostat and the Utah Paradigm. Am J Hum Biol 1998;10:599-605.

[2] Garcia-Aznar JM, Rueberg T, Doblare M. A bone remodelling model coupling microdamage growth and repair by 3D BMU-activity. Biomech Model Mechanobiol 2005; 4( (2-3)):147-67.

[3] Huiskes R, Ruimerman R, van Lenthe GH, Janssen JD. Effects of mechanical forces on maintenance and adaptation of form in trabecular bone. Nature 2000;405(6787):704-6.

[4] Huiskes R, Weinans H, Grootenboer HJ, Dalstra M, Fudala B, Slooff TJ. Adaptive boneremodeling theory applied to prosthetic-design analysis. J Biomech 1987;20(11-12):1135-50.

[5] Kuhl E, Balle F. Computational modeling of hip replacement surgery: total hip replacement vs hip resurfacing. Technische mechanik 2005;25(2):107-14.

[6] Klika V, Marsik F. A thermodynamic model of bone remodelling: the influence of dynamic loading together with biochemical control. J Musculoskelet Neuronal Interact 2010;10(3):220-30.

[7] Bougherara H, Klika V, Marsik F, Marik IA, Yahia L. New predictive model for monitoring bone remodeling. J Biomed Mater Res A 2010;95(1):9-24.

[8] Tavakkoli Avval P, Klika V, Bougherara H. Predicting bone remodeling in response to total hip arthroplasty: computational study using mechanobiochemical model. J Biomech Eng 2014;136(5):051002.

[9] Bougherara H, Bureau M, Campbell M, Vadean A, Yahia L. Design of a biomimetic polymercomposite hip prosthesis. J Biomed Mater Res A 2007;82(1):27-40.

[10] Helgason B, Perilli E, Schileo E, Taddei F, Brynjolfsson S, Viceconti M. Mathematical relationships between bone density and mechanical properties: a literature review. Clin Biomech 2008;23(2):135-46.

[11] Cristofolini L. A critical analysis of stress shielding evaluation of hip prostheses. Crit Rev Biomed Eng 1997;25(4-5):409-83.

[12] Kroger H, Venesmaa P, Jurvelin J, Miettinen H, Suomalainen O, Alhava E. Bone density at the proximal femur after total hip arthroplasty. Clin Orthop Relat Res 1998;352:66-74.

[13] Bougherara H, Bureau MN, Yahia L. Bone remodeling in a new biomimetic polymercomposite hip stem. J Biomed Mater Res A 2010;92(1):164-74.

[14] Huiskes R, Weinans H, van Rietbergen B. The relationship between stress shielding and bone resorption around total hip stem and the effects of flexible materials. Clin Orthop Relat Res $1992 ; 274$ 124-34. 
[15] Lengsfeld M, Burchard R, Gunther D, Pressel T, Schmitt J, Leppek R, et al. Femoral strain changes after total hip arthroplasty--patient-specific finite element analyses 12 years after operation. Med Eng Phys 2005;27:649-54.

[16] Turner AW, Gillies RM, Sekel R, Morris P, Bruce W, Walsh WR. Computational bone remodelling simulations and comparisons with DEXA results. J Orthop Res 2005;23(4):705-12.

[17] Carter DR, Spengler DM. Biomechanics of fracture, Bone in clinical orthopedics. 2 ed, New York: AO Publishing; 2002.

[18] Cowin S, Hegedus DH. Bone remodeling I: Theory of adaptive elasticity. J Elasticity 1976; 6:313-26.

[19] Roesler H. The history of some fundamental concepts in bone biomechanics. J Biomech 1987;20(11-12):1025-34.

[20] Wolff J. Das Gesetz der Transformation der Knochen. Berlin: Hirchwald 1892. Translated by Manquet P., Furlong R. as The Law of Bone Remodeling. Berlin: Springer; 1986.

[21] Callaghan JJ, Dysart SH, Savory CF, Hopkinson WJ. Assessing the results of hip replacement. A comparison of five different rating systems. J Bone Joint Surg Br B 1990;72(6):1008-9.

[22] Hirotsugu O, Yoshinorik K, Yukihidem M, Hidetomi T, Yoshiki Y. Outcome of Second Revision THR. Jpn J Rheum Joint Surg 2000;18:199-204.

[23] Bobyn JD, Glassman AH, Goto H, Krygier JJ, Miller JE, Brooks CE. The effect of stem stiffness on femoral bone resorption after canine porous-coated total hip arthroplasty. Clin Orthop Relat Res 1990;(261):196-213.

[24] Sumner DR, Galante JO. Determinants of stress shielding: design versus materials versus interface. Clin Orthop Relat Res 1992;(274):202-12.

[25] Li MG, Rohrl SM, Wood DJ, Nivbrant B. Periprosthetic changes in bone mineral density in 5 stem designs 5 years after cemented total hip arthroplasty, no relation to stem migration. J Arthroplasty 2007;22(5):689-91.

[26] Sano K, Ito K, Yamamoto K. Changes of bone mineral density after cementless total hip arthroplasty with two different stems. Int Orthop 2008;32(2):167-72.

[27] Sluimer JC, Hoefnagels NH, Emans PJ, Kuijer R, Geesink RG. Comparison of two hydroxyapatite-coated femoral stems: clinical, functional, and bone densitometry evaluation of patients randomized to a regular or modified hydroxyapatite-coated stem aimed at proximal fixation. J Arthroplasty 2006;21(3):344-52.

[28] Stukenborg-Colsman CM, von der Haar-Tran A, Windhagen H, Bouguecha A, Wefstaedt P, Lerch M. Bone remodelling around a cementless straight THA stem: a prospective dual-energy Xray absorptiometry study. Hip Int 2012;22(2):166-71.

[29] Beaupre GS, Orr TE, Carter DR. An approach for time-dependent bone modeling and remodeling-application: a preliminary remodeling simulation. J Orthop Res 1990;8(5):662-70. 
[30] Levenston ME, Carter DR. An energy dissipation-based model for damage stimulated bone adaptation. J Biomech 1998;31(7):579-86.

[31] Martin RB. Targeted bone remodeling involves BMU steering as well as activation. Bone 2007; 40:1574-80.

[32] Prendergast PJ, Taylor D. Prediction of bone adaptation using damage accumulation. J Biomech 1994;27(8):1067-76.

[33] Rouhi G, Epstein M, Sudak L, Herzog W. Modeling bone resorption using mixture theory with chemical reactions. J Mech Mater Struct 2007; 2:1141-55.

[34] Scheiner S, Pivonka P, Hellmich C. Coupling systems biology with multiscale mechanics, for computer simulations of bone remodeling. Comput Method Appl M 2013;254:181-96.

[35] Weinans H, Huiskes R, Grootenboer HJ. The behavior of adaptive bone-remodeling simulation models. Journal of Biomechanics 1992;25(12):1425-41.

[36] Hernandez CJ, Beaupre GS, Carter DR. A model of mechano-biologic and metabolic Influences bone adaptation. J Rehabil Res Dev 2000;37:235-44.

[37] Hernandez CJ, Beaupre GS, Keller TS, Carter DR. The influence of bone volume fraction and ash fraction on bone strength and modulus. Bone 2001;29(1):74-8.

[38] Ruimerman R, Huiskes R, van Lenthe GH, Janssen JD. A computer-simulation model relating bone-cell metabolism to mechanical adaptation of trabecular architecture. Computer Methods in Biomechanics and Biomedical Engineering 2001;4(5):433-48.

[39] Kroll MH. Parathyroid hormone temporal effects on bone formation and resorption. B Math Biol 2000;62:163-88.

[40] Rattanakul C, Lenbury Y, Krishnamara N, Wollkind DJ. Modeling of bone formation and resorption mediated by Parathyroid hormone: Response to Estrogen/PTH therapy. Biosystems 2003;70:55-72.

[41] Komarova SV, Smith RJ, Dixon SJ, Sims SM, Wahl LM. Mathematical model predicts a critical role for Osteoclast autocrine regulation in the control of bone remodeling. Bone 2003;33:206-15.

[42] Lemaire V, Tobin FL, Greller LD, Cho CR, Suva LJ. Modeling the Interactions between Osteoblast and Osteoclast activities in bone remodeling. J Theor Biol 2004;229:293-309.

[43] Sayyidmousavi A. Application of a thermodynamic based model to investigate bone remodeling after total hip arthroplasty using different hip implants. Ryerson University, Masters Thesis; 2010.

[44] Michaelis L, Menten ML. Kinetik der invertinwirkung. Biochem Z 1913;49:333-69.

[45] Lewis JL, Askew MJ, Wixson RL, Kramer GM, Tarr RR. The influence of prosthetic stem stiffness and of a calcar collar on stresses in the proximal end of the femur with a cemented femoral component. J Bone Joint Surg Am 1984;66(2):280-6. 
[46] Djerf K, Gillquist J. Calcar unloading after hip replacement. A cadaver study of femoral stem designs. Acta Orthop Scand 1987;58(2):97-103.

[47] Maistrelli GL, Fornasier V, Binnington A, McKenzie K, Sessa V, Harrington I. Effect of stem modulus in a total hip arthroplasty model. J Bone Joint Surg Br 1991;73(1):43-6.

[48] Hedia HS. Stiffness optimisation of cement and stem materials in total hip replacement. Biomed Mater Eng 2001;11(1):1-10.

[49] Evans SL, Gregson PJ. Numerical optimization of the design of a coated, cementless hip prosthesis. Journal of Materials Science: Materials in Medicine 1994;5(8):507-10.

[50] Ali MS, French TA, Hastings GW, Rae T, Rushton N, Ross ER, et al. Carbon fibre composite bone plates. Development, evaluation and early clinical experience. J Bone Joint Surg $\mathrm{Br}$ 1990;72(4):586-91.

[51] Erkmen E, Meric G, Kurt A, Tunc Y, Eser A. Biomechanical comparison of implant retained fixed partial dentures with fiber reinforced composite versus conventional metal frameworks: a 3D FEA study. J Mech Behav Biomed Mater 2011;4(1):107-16.

[52] Feerick EM, Kennedy J, Mullett H, FitzPatrick D, McGarry P. Investigation of metallic and carbon fibre PEEK fracture fixation devices for three-part proximal humeral fractures. Med Eng Phys 2013;35(6):712-22.

[53] Fujihara K, Huang ZM, Ramakrishna S, Satknanantham K, Hamada H. Performance study of braided carbon/PEEK composite compression bone plates. Biomaterials 2003;24(15):2661-7.

[54] Hastings GW. Carbon fibre composites for orthopaedic implants. Composites 1978;9(3):1937.

[55] Woo SL, Akeson WH, Levenetz B, Coutts RD, Matthews JV, Amiel D. Potential application of graphite fiber and methyl methacrylate resin composites as internal fixation plates. J Biomed Mater Res 1974;8(5):321-38.

[56] Samiezadeh S, Tavakkoli Avval P, Fawaz Z, Bougherara H. Biomechanical assessment of composite versus metallic intramedullary nailing system in femoral shaft fractures: a finite element study. Clin Biomech 2014;(DOI: 10.1016/j.clinbiomech.2014.05.010).

[57] Akhavan S, Matthiesen MM, Schulte L, Penoyar T, Kraay MJ, Rimnac CM, et al. Clinical and histologic results related to a low-modulus composite total hip replacement stem. J Bone Joint Surg Am 2006;88(6):1308-14.

[58] Katoozian H, Davy DT, Arshi A, Saadati U. Material optimization of femoral component of total hip prosthesis using fiber reinforced polymeric composites. Medical Engineering and Physics 2001;23(7):503-9.

[59] Mendes DG, Roffman M, Soudry M, Angel D, Boss J, Charit Y, et al. A composite hip implant. Orthop Rev 1988;17(4):402-7. 
[60] Sridhar I, Adie PP, Ghista DN. Optimal design of customised hip prosthesis using fiber reinforced polymer composites. Materials and Design 2010;31(6):2767-75.

[61] Li C, Granger C, Del Schutte Jr H, Biggers Jr SB, Kennedy JM, Latour Jr RA. Failure analysis of composite femoral components for hip arthroplasty. Journal of Rehabilitation Research and Development 2003;40(2):131-45.

[62] Lim JW, Jeong JY, Ha SK. Design of composite hip prostheses considering the long-term behavior of the femur. JSME International Journal, Series C: Mechanical Systems, Machine Elements and Manufacturing 2003;46(3):991-9.

[63] Janes GC, Collopy DM, Price R, Sikorski JM. Bone density after rigid plate fixation of tibial fractures. A dual-energy X-ray absorptiometry study. J Bone Joint Surg Br 1993;75(6):914-7.

[64] Pilliar RM, Cameron HU, Binnington AG, Szivek J, Macnab I. Bone ingrowth and stress shielding with a porous surface coated fracture fixation plate. J Biomed Mater Res 1979;13(5):799810.

[65] Terjesen T, Nordby A, Arnulf V. Bone atrophy after plate fixation. Computed tomography of femoral shaft fractures. Acta Orthop Scand 1985;56(5):416-8.

[66] Rockwood CA, Bucholz RW, Green DP, Court-Brown CM, Heckman JD, Tornetta P. Rockwood and Green's Fractures in Adults. Wolters Kluwer Health/Lippincott Williams \& Wilkins; 2010.

[67] IMG Buddy; Bone Structure. Access date: 2015-05-07; Available from: http://imgbuddy.com/bone-structure.asp.

[68] Ni GX, Lu WW, Chiu KY, Fong DY. Cemented or uncemented femoral component in primary total hip replacement? A review from a clinical and radiological perspective. J Orthop Surg (Hong Kong) 2005;13(1):96-105.

[69] Glassgow University; Compact and Spongy bone. Access date: 2015-05-07; Available from: http://www.gla.ac.uk/t4/ fbls/files/fab/tutorial/generic/bone2.html.

[70] Shibuya B. What Causes Osteoporosis. Access date: 2015-05-07; Available from: http://drshibuya.com/osteo-patho.html.

[71] Bagheri Z. Biomechanical development and analysis of a new carbon fiber/flax/epoxy plate for repairing femur fractures. Ryerson University, PhD Thesis; 2014.

[72] Kim SYR. Biomechanical evaluation of periprosthetic femoral fractre fixation. Ryerson University, Masters Thesis; 2010.

[73] Larsen A. Growing bones and crossfit kids: The biology every kids coach shoud know. Access date: 2015-05-04; Available from: http://breakingmuscle.com/family-kids/growing-bones-andcrossfit-kids-the-biology-every-kids-coach-should-know.

[74] Raisz LG. Pathogenesis of osteoporosis: concepts, conflicts, and prospects. J Clin Invest 2005;115(12):3318-25. 
[75] Cullinance D, Einhorn T. Biomechanics of Bone, Principles of Bone Biology. 2 ed. Vol. 1. San Diego: : Academic Press; 2002.

[76] Thakur A. The Elements of Fracture Fixation. 2 ed, New Delhi: Reed Elsevier India Private Limited; 2007.

[77] Pankovich A, Davenport K. Fractures of the Femoral Shaft, Handbook of Fractures. New York: McGraw-Hill; 2006.

[78] Sanders R. Trauma: Core knowledge in orthopaedics. Philadelphia: Mosby, Inc., an affiliate of Elsevier Inc; 2008.

[79] Simon R, Koenigsknecht S. Fractures of the Distal Femur, Emergency orthopedics: the extremities. 4 ed, New York: McGraw-Hill; 2001.

[80] Frankel V, Nordin M. Basic Biomechanics of the Skeletal System. London: Kimpton Publishers; 1980.

[81] Siddiqui F. Biomechanical Evaluation of a Carbon Fibre Epoxy Composite Plate in an Injured and Healed Femur Using Infrared Thermography and Finite Element Analysis. Ryerson University, Masters Thesis; 2012.

[82] Tavakkoli Avval P, Samiezadeh S, Bougherara H. Long-term Response of Femoral Density to Hip Implant and Bone Fracture Plate: a Computational Study using Mechano-Biochemical Model. Submitted to MED ENG PHYS 2015.

[83] Tavakkoli Avval P, Samiezadeh S, Klika V, Bougherara H. Investigating Stress Shielding Spanned by Biomimetic Polymer-Composite vs. Metallic Hip Stem: A Computational Study using Mechano-biochemical Model. J Mech Behav Biomed Mater 2014:http://dx.doi.org/10.1016/j.jmbbm.2014.09.019.

[84] Samiezadeh S, Tavakkoli Avval P, Fawaz Z, Bougherara H. An Effective Approach for Optimization of a Composite Intramedullary Nail for Treating Femoral Shaft Fractures. Submitted to J Bio Mech 2015.

[85] Cheung G, Zalzal P, Bhandari M, Spelt JK, Papini M. Finite element analysis of a femoral retrograde intramedullary nail subject to gait loading. Medical Engineering \& Physics 2004;26(2):93-108.

[86] Bitsakos C, Kerner J, Fisher I, Amis AA. The effect of muscle loading on the simulation of bone remodelling in the proximal femur. J Biomech 2005;38(1):133-9.

[87] Bagheri ZS, Tavakkoli Avval P, Bougherara H, Aziz MS, Schemitsch EH, Zdero R. Biomechanical analysis of a new carbon fiber/flax/epoxy bone fracture plate shows less stress shielding compared to a standard clinical metal plate. J Biomech Eng 2014;136(9).

[88] Dubov A, Kim SY, Shah S, Schemitsch EH, Zdero R, Bougherara H. The biomechanics of plate repair of periprosthetic femur fractures near the tip of a total hip implant: the effect of cablescrew position. Proc Inst Mech Eng H 2011;225(9):857-65. 
[89] Shah S, Kim SY, Dubov A, Schemitsch EH, Bougherara H, Zdero R. The biomechanics of plate fixation of periprosthetic femoral fractures near the tip of a total hip implant: cables, screws, or both? Proc Inst Mech Eng H 2011;225(9):845-56.

[90] ANSYS Help V 14.5. Pennsylvania: ANSYS Inc; 2012.

[91] Papini M, Zdero R, Schemitsch EH, Zalzal P. The biomechanics of human femurs in axial and torsional loading: comparison of finite element analysis, human cadaveric femurs, and synthetic femurs. J Biomech Eng 2007;129(1):12-9.

[92] Balle F. Biomechanische untersuchungen zur Knochen-implantat-interaktion mit Hilfe der methode der finite elemente. University of Kaiserslautern; 2004.

[93] Behrens BA, Nolte I, Wefstaedt P, Stukenborg-Colsman C, Bouguecha A. Numerical investigations on the strain-adaptive bone remodelling in the periprosthetic femur: influence of the boundary conditions. Biomed Eng Online 2009;8:7.

[94] Lerch M, Kurtz A, Stukenborg-Colsman C, Nolte I, Weigel N, Bouguecha A, et al. Bone remodeling after total hip arthroplasty with a short stemmed metaphyseal loading implant: finite element analysis validated by a prospective DEXA investigation. J Orthop Res 2012;30(11):18229.

[95] Hodgskinson R, Currey JD. The effect of variation in structure on the Young's modulus of cancellous bone: a comparison of human and non-human material. Proc Inst Mech Eng H 1990;204(2):115-21.

[96] Hoiberg M, Nielsen TL, Wraae K, Abrahamsen B, Hagen C, Andersen M, et al. Populationbased reference values for bone mineral density in young men. Osteoporos Int 2007;18(11):150714.

[97] Truong LH, Kuliwaba JS, Tsangari H, Fazzalari NL. Differential gene expression of bone anabolic factors and trabecular bone architectural changes in the proximal femoral shaft of primary hip osteoarthritis patients. Arthritis Res Ther 2006;8(6):R188.

[98] Gruen TA, McNeice GM, Amstutz HC. Modes of failures of cemented stem-type femoral components. Clin Orthop Relat Res 1979;141:17-27.

[99] Trevisan C, Bigoni M, Randelli G, Marinoni EC, Peretti G, Ortolani S. Periprosthetic bone density around fully hydroxyapatite coated femoral stem. Clin Orthop Relat Res 1997;(340):10917.

[100] Wixson RL, Stulberg SD, Van Flandern GJ, Puri L. Maintenance of proximal bone mass with an uncemented femoral stem analysis with dual-energy x-ray absorptiometry. J Arthroplasty 1997;12(4):365-72.

[101] Niinimaki T, Jalovaara P. Bone loss from the proximal femur after arthroplasty with an isoelastic femoral stem. BMD measurements in 25 patients after 9 years. Acta Orthop Scand 1995;66(4):347-51. 
[102] Shim VB, Pitto RP, Anderson IA. Quantitative CT with finite element analysis: towards a predictive tool for bone remodelling around an uncemented tapered stem. Int Orthop 2012;36(7):1363-9.

[103] Glassman AH, Crowninshield RD, Schenck R, Herberts P. A low stiffness composite biologically fixed prosthesis. Clin Orthop Relat Res 2001;(393):128-36.

[104] Frost HM. Skeletal structural adaptations to mechanical usage (SATMU): 2. Redefining Wolff's law: the remodeling problem. Anat Rec 1990;226(4):414-22.

[105] Kilgus DJ, Shimaoka EE, Tipton JS, Eberle RW. Dual-energy X-ray absorptiometry measurement of bone mineral density around porous-coated cementless femoral implants. Methods and preliminary results. J Bone Joint Surg Br 1993;75(2):279-87.

[106] Skoldenberg OG, Boden HS, Salemyr MO, Ahl TE, Adolphson PY. Periprosthetic proximal bone loss after uncemented hip arthroplasty is related to stem size: DXA measurements in 138 patients followed for 2-7 years. Acta Orthop 2006;77(3):386-92.

[107] Kroger H, Miettinen H, Arnala I, Koski E, Rushton N, Suomalainen O. Evaluation of periprosthetic bone using dual-energy $\mathrm{x}$-ray absorptiometry: precision of the method and effect of operation on bone mineral density. J Bone Miner Res 1996;11(10):1526-30.

[108] Kroger H, Venesmaa P, Jurvelin J, Miettinen H, Suomalainen O, Alhava E. Bone density at the proximal femur after total hip arthroplasty. Clin Orthop Relat Res 1998;(352):66-74.

[109] Yamaguchi K, Masuhara K, Ohzono K, Sugano N, Nishii T, Ochi T. Evaluation of periprosthetic bone-remodeling after cementless total hip arthroplasty. The influence of the extent of porous coating. J Bone Joint Surg Am 2000;82-A(10):1426-31.

[110] Wang C, Wang LZ, Fan YB.2013, Long-term Prediction of Bone Density Distribution for Retained Intramedullary Nail, in World Congress on Medical Physics and Biomedical Engineering May 26-31, 2012, Beijing, China, M Long, Editor, Springer Berlin Heidelberg: P ace Published. p. $161-4$.

[111] Allen JC, Jr., Lindsey RW, Hipp JA, Gugala Z, Rianon N, LeBlanc A. The effect of retained intramedullary nails on tibial bone mineral density. Clin Biomech (Bristol, Avon) 2008;23(6):83943.

[112] Tennyson RC, Ewert R, Niranjan V. Dynamic viscoelastic response of bone. Experimental Mechanics 1972;12(11):502-7. 